Check for updates

Cite this: Mater. Adv., 2021, 2, 7139

Received 20th July 2021,

Accepted 28th September 2021

DOI: $10.1039 / \mathrm{d} 1 \mathrm{ma} 00630 \mathrm{~d}$

rsc.li/materials-advances

\section{From metal-organic framework powders to shaped solids: recent developments and challenges}

\author{
Bakytzhan Yeskendir, ${ }^{\text {ab }}$ Jean-Philippe Dacquin, (D) ${ }^{a}$ Yannick Lorgouilloux, (DD ${ }^{b}$ \\ Christian Courtois, id b Sébastien Royer (iD a and Jérémy Dhainaut iD *a
}

\begin{abstract}
Metal-organic frameworks represent a class of porous materials which have developed considerably over the past few years. Their highly porous structure makes them outperform conventional adsorbents in hot topics such as dihydrogen and methane storage, and carbon dioxide capture. Their consequent modularity, based on the assembly of organic linkers and metal ions or clusters, also brings novel perspectives in catalysis, sensing and drug delivery just to name a few. However, one of the main bottlenecks to their broader use remains their shaping. Especially, shaped materials should present long-term mechanical stability as well as preserve their physical and chemical properties. This makes shaping of MOFs a special case as their thermal and chemical stabilities remain a downside as compared to other traditional porous materials such as silicas and zeolites today. Therefore, ever-increasing efforts have been devoted to the shaping of these materials. In this review, the state of the art for the preparation of shaped 3D MOF-based materials will be presented. Emphasis will be given to the final physical and chemical properties of the shaped solids comparatively to the initial powders, when data are available. In the first part, traditional techniques based on applying a significant force to MOF-based powders will be reviewed. These include pelletization, granulation, and extrusion, which generally lead to an increase of the final volumetric gas uptake of the objects. At the same time, the advantages and disadvantages of each technique will be discussed as well as the main outcome on the final objects. In the second part, the focus will be on newly-emerging techniques such as 3D printing and spray drying. The former also maximizes the volumetric gas uptake of the final materials, and for both techniques the quality of the final objects heavily relies on the working parameters. Finally, the third part will include the so-called "phase separation" shaping techniques which are for the most part performed without using special techniques. This implies shaping via physical and chemical phenomena such as sublimation and precipitation. Subsequently, a discussion on the performance of these materials for adsorption-based applications will be provided. Finally, perspectives and future outlook will be discussed.
\end{abstract}

\section{Introduction}

Metal-Organic Frameworks (MOFs; also called Porous Coordination Polymers, PCPs) have attracted a great deal of attention since they were first described in 1995 and developed in the early 2000s. ${ }^{1,2}$ They represent a new class of hybrid crystalline microporous materials as they are composed of metal nodes (ions or clusters) bound together by multitopic organic linkers. Such coordination allows for an eventual 1-, 2- or 3-dimensional

\footnotetext{
${ }^{a}$ Univ. Lille, CNRS, Centrale Lille, Univ. Artois, UMR 8181 - UCCS - Unité de Catalyse et Chimie du Solide, F-59000 Lille, France.E-mail: jeremy.dhainaut@univlille.fr

${ }^{b}$ Université Polytechnique Hauts-de-France, EA 2443 - LCMPA, 59600 Maubeuge, France
}

framework. In most cases, the metal core is formed from transition metals, while the linker is often comprised of cyclic organic compounds presenting carboxylate groups or N-donors. Over the past decades, researchers from all over the world have probed a significant number of different combinations of metal precursors, organic linkers, and synthesis conditions, leading to the discovery of many new MOF structures. Nowadays, this denomination includes several thousand structures including the famous HKUST- $1,{ }^{3}$ UiO- $66,{ }^{4}$ ZIF- $8{ }^{5}$ and MIL- $101 .{ }^{6}$

Owing to their low density and exceptionally high surface area, MOFs have found an initial application as adsorbents in gas separation and storage. Indeed, the available surface area of MOFs can exceed multiple times those of the most well-known porous materials such as zeolites, silicas and activated carbons. According to numerous studies, MOFs outperform most 
benchmark adsorbents in terms of gas adsorption ${ }^{7}$. Moreover, MOFs can be subjected to structural modifications by varying the metal node and/or the organic linker via in situ or postsynthesis treatments. This, in turn, implies the textural and chemical tunability of MOFs and therefore expands their application to catalysis, ${ }^{8}$ drug-delivery, ${ }^{9}$ sensing, ${ }^{10}$ water purification, ${ }^{11}$ and energy storage, ${ }^{12}$ just to name a few. Besides, MOFs have been shown to be excellent templates for the production of high-performance materials, following mainly pyrolysis or chemical leaching. A wide variety of MOFderived nanostructured materials can be obtained, including porous metal oxides, carbides, phosphides, carbons, doped carbons and composites. ${ }^{13}$ The unique chemical compositions and pore architectures inherited from the parent MOFs make these materials suitable for unique applications in catalysis ${ }^{14}$ and energy storage. ${ }^{15-17}$

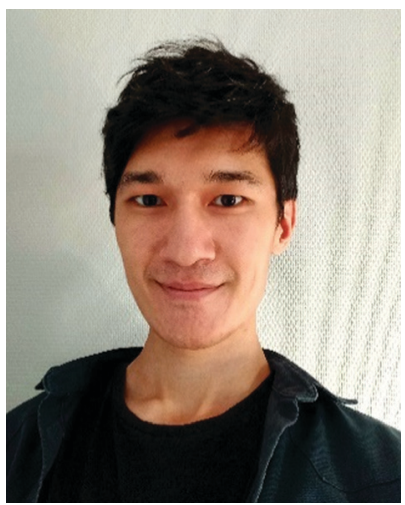

Bakytzhan Yeskendir
Bakytzhan Yeskendir received his MSc in Chemistry and Spectroscopy in 2018 within the framework of the Advanced Spectroscopy in Chemistry Master Program funded by Erasmus Mundus. He is now pursuing his $P h D$ in materials science in the fields of synthesis, characterization, upscaling and shaping of materials for application in catalysis and gas adsorption, with special interest in the design of catalysts and adsorbents based on Metal-Organic Frameworks and zeolites, as collaborating work between the MATCAT group led by Pr. Sébastien Royer at the Universite de Lille and the LMCPA led by Pr. Christian Courtois at UPHF.
Typically, MOFs are produced in polycrystalline powder form, with the size of individual crystals ranging from several tens of nanometers to a few microns. Continuous studies on synthesis optimization and product characterization have stimulated the production of MOFs on a larger scale. Thus, a number of them are now commercially available and provided by BASF (HKUST-1/Basolite C300, ZIF-8/Basolite Z1200, Fe-BTC/ Basolite F300), Strem Chemicals (CAU-10, MIL-53(Al), MIL101(Al), PCN-250(Fe), UiO-66), and others.

Usually, powders are not convenient to use in big industrial reactors as they cause several difficulties from the processing point of view. They induce, for example, consequent pressure drops within the reactor bed. In addition to that, powders can induce clogging, and it is generally complicated to handle and recover them, as they involve additional costly separation steps. In order to overcome these issues, MOF powders should be

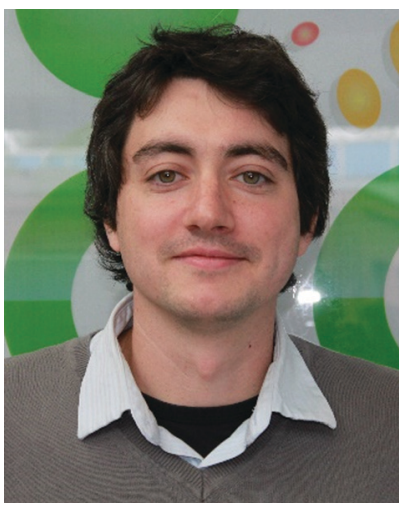

Jean-Philippe Dacquin
Jean-Philippe Dacquin obtained his $\mathrm{PhD}$ from the Université Sciences et Technologies de Lille 1 (France) in 2008. After two postdoctoral years at the Cardiff Catalysis Institute following the University of York with Karen Wilson and Adam F. Lee, he returned to the University of Lille where he holds a position of Associate Professor. He's the administrative head of the bachelor of Chemistry and teaches courses on inorganic chemistry and analytical chemistry. His research is devoted to the preparation of solid catalysts with controlled porosity and their application in environmental catalysis.

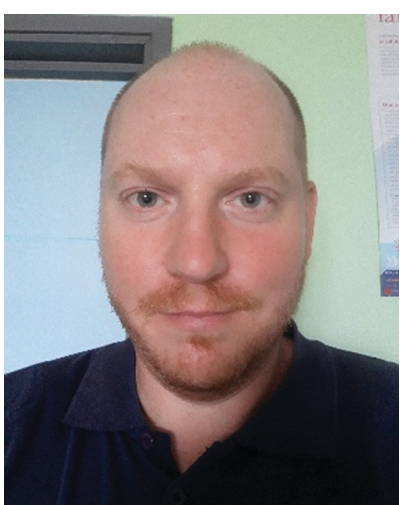

Yannick Lorgouilloux
Yannick Lorgouilloux was born in France in 1981. He graduated from the University of Limoges, France, with a master's degree in Industrial Ceramics in 2004. After working for 6 years on powder synthesis for heterogeneous catalysis (PhD in 2007 in Mulhouse, France, and 3 years of post-doc in Leuven, Belgium), he is now an Associate Professor at the Polytechnic University Hauts-de-France. His main research activities deal with the control of the porosity and microstructure of ceramic materials for various applications, such as piezoelectric materials, transparent ceramics and hierarchically porous catalytic monoliths.

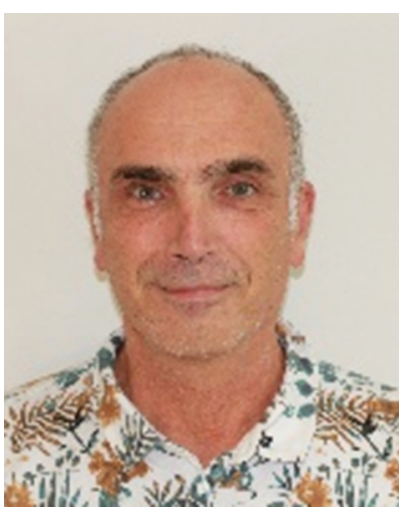

Christian Courtois
Christian Courtois was born in France in 1963. He graduated from the University of Limoges, France, with a PhD degree in Ceramics and Surface Treatments in 1992. Since 1993 as Associate Professor then Professor at the Université Polytechnique Hauts de France, his research deals with powder synthesis, and control of the microstructure of ceramic materials for various applications, mainly for piezoelectric and other electric materials, and hierarchically porous catalytic monoliths. 
Table 1 Shaped bodies for different types of reactors. Adapted from ref. 18

\begin{tabular}{|c|c|c|c|}
\hline $\begin{array}{l}\text { Shaping } \\
\text { technique }\end{array}$ & $\begin{array}{l}\text { Object dimensions } \\
(\mathrm{mm})\end{array}$ & Shape & Type of reactor \\
\hline Pelletization & $\begin{array}{l}d=3-15 \\
h=3-15\end{array}$ & Pellets & Fixed bed reactor \\
\hline Extrusion & $\begin{array}{l}d=1-50 \\
h=3-30\end{array}$ & Extrudates & Fixed bed reactor \\
\hline Granulation & $d=1-20$ & Beads & Fixed bed reactor \\
\hline Spray-drying & $d=0.02-0.2$ & Microspheres & $\begin{array}{l}\text { Fluidized bed } \\
\text { reactor }\end{array}$ \\
\hline
\end{tabular}

formulated into packed objects with defined size and dimensions. This process is called "shaping" and generally represents the compaction of individual crystallites into millimeter-sized objects. Shaping allows for production of easily-handled objects required for industrial processes and generally has a few objectives:

(1) formulation of powders into mechanically stable objects to withstand a variety of process conditions (elevated pressure, gas/liquid flow, mixing);

(2) reduction of mass transfer limitations within the reactor volume; and

(3) preservation of the physico-chemical properties of the pristine powder.

The latter represents the major challenge especially for MOFs as they are prone to drastic losses in terms of available surface area and pore volume upon the formulation process. Nowadays, there are plenty of shaping techniques available which enable formulation of powders into shaped bodies for use in different types of reactors (Table 1).

Therefore, each of the shaping techniques provides unique features to the final objects in terms of size and appearance for a defined application. This review will focus on conventional shaping techniques such as granulation, pelletization, extrusion, and spray-drying and challenges associated upon

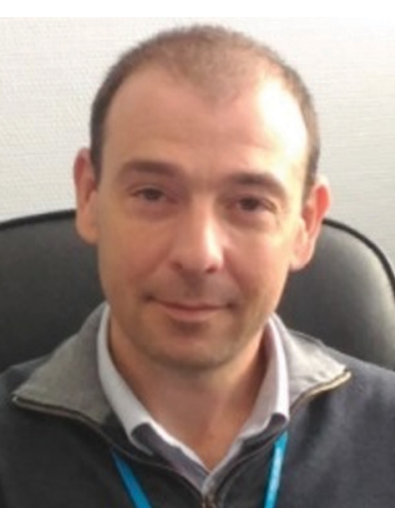

Sébastien Royer
Sébastien Royer obtained his PhD in Chemical Engineering from Laval University - $Q C-C A$ in 2004. Since 2015, he has been Full Professor at the University of LilleFR. He leads the MATCAT research group from UCCS laboratory as well as the UPCAT technological platform devoted to the study of catalyst scale-up (up to $10 \mathrm{~kg}$ ) and shaping. S. Royer has done research in the fields of catalytic materials engineering, with interest in control of porosity (mesoporous, macroporous, and hierarchical porosity), dispersion and stability of metallic elements, long term stability and regeneration procedures, characteristics of shaped catalysts. formulation of MOF powders. This will also include the 3D printing method as it can be referred to as a type of extrusion with controlled deposition of the forming paste in three dimensions in space. Therefore, 3D printing allows shaping powders with desired shapes and dimensions for a wide variety of applications. They will be as well discussed in the corresponding section along with the challenges related to the formulation of MOF powders. Besides, a separate section will be dedicated to the so-called non-conventional techniques which include freeze granulation, ice templating and biopolymer precipitation. Membranes and coatings, and sol-gel-based monoliths have been excluded from this review on purpose as they have been recently reviewed. ${ }^{19}$

This review includes discussions on the specifics of each shaping technique such as the influence of pressure on raw powders and their textural properties upon pelletization, or the appropriate choice of a binder for granulation and extrusion. Working parameters for 3D printing and spray-drying will be considered as well. Moreover, special cases where certain MOFs are not eligible for being shaped by one or another technique will be highlighted. This depends on the nature of the parent MOF determined by the coordination state of the metal and the organic ligand. Consequently, to date, shaping has been mostly applied to a few MOF structures summarized in Table 2.

\section{Pelletization}

Pelletization (also referred to as "pressing”, "compressing” or "tableting") is the most widely used shaping technique and represents the compaction of powder particles by applying pressure. Owing to its simplicity, pelletization is the first shaping technique to have been applied on an industrial scale. Overall, this method implies pressing powder in a die by applying a uniaxial pressure of several tenths of MPa with a

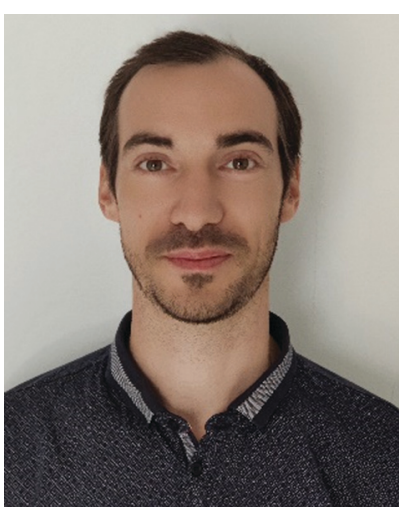

Jérémy Dhainaut
Jérémy Dhainaut received his $P h D$ in Chemistry of Materials from the University of Upper Alsace (Mulhouse) in 2012. He further developed an expertise in the fields of porous materials and their shaping through postdoc positions at the Ceramic Synthesis and Functionalisation Laboratory (Cavaillon), the Institute of Research on Catalysis and the Environment (Lyon), the Laboratory of Catalysis and Solid-State Chemistry (Villeneuve d'Ascq), and the Institute for Integrated Cell-Material Sciences (Kyoto). In 2019, he was appointed as a CNRS Researcher at UCCS. His work focuses on studying the effect of shaping methods on the physicochemical properties of porous materials including MOFs. 
Table 2 Schematic representation of some of the reviewed MOF structures

\begin{tabular}{|c|c|c|c|c|}
\hline MOF & Space group & Metal cluster & Organic linker & Framework \\
\hline ZIF-8 & $I \overline{4} 3 m$ & $\mathrm{Zn}^{2+}$ & Imidazolate & \\
\hline MOF-74 & $R \overline{3}$ & $\mathrm{Ni}^{2+}, \mathrm{Co}^{2+}, \mathrm{Mg}^{2+}$ & $\begin{array}{l}\text { 2,5-dihydroxybenzene-1,4- } \\
\text { dicarboxylate }\end{array}$ & \\
\hline MIL-53 & Imma & $\mathrm{Al}^{3+}$ & Benzene-1,4-dicarboxylate & \\
\hline UiO-66 & $F m \overline{3} m$ & $\mathrm{Zr}^{4+}$ & Benzene-1,4-dicarboxylate & \\
\hline
\end{tabular}

punch (Fig. 1). Some configurations are based on both upper and lower punches moving towards each other; however, in most configurations one punch remains stationary. In order to achieve maximum compaction, the punches may be left in the compression position for a certain time before recovering the final shaped object. Typically, this process yields cylinder or oblong-type shaped bodies which are called "pellets" or "tablets".

The process parameters entirely depend on the initial powder to be shaped. Mainly, the pressure applied on it should be carefully selected to avoid the complete destruction of the crystal structure (amorphization) and therefore loss of intrinsic
Die filling

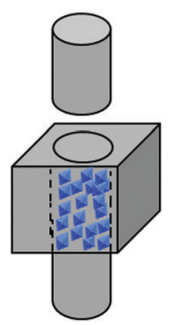

Compression
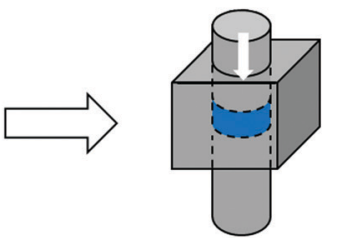

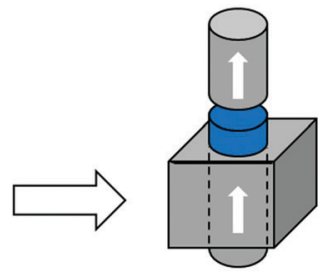

MOF pellet
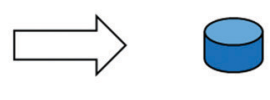

Fig. 1 Schematic representation of the pelletization process applied to polycrystalline MOF powder. 
Table 3 Effect of the pelletization process on the textural properties of MOFs

\begin{tabular}{|c|c|c|c|c|c|c|}
\hline \multirow[b]{2}{*}{ MOF } & \multirow[b]{2}{*}{ Metal/linker } & \multirow[b]{2}{*}{ Binder, wt $\%$} & \multirow[b]{2}{*}{ Applied pressure, $\mathrm{MPa}$} & \multicolumn{2}{|c|}{$S_{\mathrm{BET}}, \mathrm{m}^{2} \mathrm{~g}^{-1}$} & \multirow[b]{2}{*}{ Ref. } \\
\hline & & & & Powder & Pellet & \\
\hline MOF-5 & $\mathrm{Zn} / \mathrm{BDC}$ & None & 180 & 2160 & $275(-87 \%)$ & 25 \\
\hline MOF-5 & $\mathrm{Zn} / \mathrm{BDC}$ & None & 10.3 & 3450 & $6(-99 \%)$ & 26 \\
\hline MOF-5 & $\mathrm{Zn} / \mathrm{BDC}$ & None & 2 & 2777 & $1450(-48 \%)$ & 27 \\
\hline MOF-5 & $\mathrm{Zn} / \mathrm{BDC}$ & None & 80 & 2762 & $\sim 1600(-42 \%)$ & 28 \\
\hline MOF-177 & $\mathrm{Zn} / \mathrm{BTB}$ & None & - & 4100 & $3300(-20 \%)$ & 32 \\
\hline MIL-53 & $\mathrm{Al} / \mathrm{BDC}$ & PVA, 13 & None & - & - & 23 \\
\hline MIL-53 & $\mathrm{Al} / \mathrm{BDC}$ & - & - & 830 & $601(-28 \%)$ & 34 \\
\hline MIL-53 & $\mathrm{Al} / \mathrm{BDC}$ & - & - & - & 560 & 35 \\
\hline MIL-53 & $\mathrm{Al} / \mathrm{BDC}$ & None & 125 & 796 & $538(-32 \%)$ & 37 \\
\hline ZIF-8 & $\mathrm{Zn} / \mathrm{MIM}$ & None & 125 & 1983 & $1736(-12 \%)$ & 37 \\
\hline ZIF-8 & $\mathrm{Zn} / \mathrm{MIM}$ & None & 300 & 1774 & $1551(-13 \%)$ & 38 \\
\hline ZIF-8 & $\mathrm{Zn} / \mathrm{MIM}$ & None & 229 & 1433 & $1278(-11 \%)$ & 39 \\
\hline ZIF-8 & $\mathrm{Zn} / \mathrm{MIM}$ & Talc & 24 & 1187 & $951(-20 \%)$ & 40 \\
\hline ZIF-8 & $\mathrm{Zn} / \mathrm{MIM}$ & SB & 24 & 1187 & $959(-19 \%)$ & 40 \\
\hline SIM-1 & $\mathrm{Zn} / \mathrm{MIC}$ & None & 400 & 516 & $370(-28 \%)$ & 39 \\
\hline MIL-100 & $\mathrm{Fe} / \mathrm{BTC}$ & Graphite, 3 & 34 & 2088 & $1300(-38 \%)$ & 57 \\
\hline MIL-101 & $\mathrm{Cr} / \mathrm{BDC}$ & None & - & 3623 & $2602(-28 \%)$ & 41 \\
\hline MIL-101 & $\mathrm{Cr} / \mathrm{BDC}$ & None & - & 3677 & $3302(-10 \%)$ & 43 \\
\hline MIL-101 & $\mathrm{Cr} / \mathrm{BDC}$ & None & - & 3414 & $2745(-20 \%)$ & 42 \\
\hline MIL-101 & $\mathrm{Cr} / \mathrm{BDC}$ & Al tapes ${ }^{a}$ & - & 3814 & $2955(-23 \%)$ & 44 \\
\hline MIL-101 & $\mathrm{Cr} / \mathrm{BDC}$ & Graphite, 3 & 34 & 4066 & $2660(-35 \%)$ & 57 \\
\hline CPO-27 & Ni/DHBDC & None & 100 & 1060 & $1050(-1 \%)$ & 45 \\
\hline MOF-74 & $\mathrm{Mg} / \mathrm{DHBDC}$ & None & 30 & 1249 & $1034(-17 \%)$ & 46 \\
\hline HKUST-1 & $\mathrm{Cu} / \mathrm{BTC}$ & None & 180 & 1145 & $470(-59 \%)$ & 25 \\
\hline HKUST-1 & $\mathrm{Cu} / \mathrm{BTC}$ & None & 69 & 1698 & $892(-47 \%)$ & 47 \\
\hline HKUST-1 & $\mathrm{Cu} / \mathrm{BTC}$ & None & 5 & 1737 & $1007(-42 \%)$ & 48 \\
\hline HKUST-1 & $\mathrm{Cu} / \mathrm{BTC}$ & None & 121 & 1288 & $1091(-15 \%)$ & 49 \\
\hline HKUST-1 & $\mathrm{Cu} / \mathrm{BTC}$ & Graphite, 2 & 112 & 1288 & $949(-26 \%)$ & 49 \\
\hline HKUST-1 & $\mathrm{Cu} / \mathrm{BTC}$ & None & 24 & 1897 & $1377(-27 \%)$ & 39 \\
\hline HKUST-1 & $\mathrm{Cu} / \mathrm{BTC}$ & None & 400 & 1897 & $476(-75 \%)$ & 39 \\
\hline UiO-66 & $\mathrm{Zr} / \mathrm{BDC}$ & None & 69 & 1080 & $1090(=)$ & 47 \\
\hline UiO-66 & $\mathrm{Zr} / \mathrm{BDC}$ & Graphite, 1 & - & 1140 & $885(-22 \%)$ & 52 \\
\hline UiO-66 & $\mathrm{Zr} / \mathrm{BDC}$ & None & 18 & 1426 & $1459(-)$ & 49 \\
\hline UiO-66 & $\mathrm{Zr} / \mathrm{BDC}$ & None & 665 & 1737 & $1707(-2 \%)$ & 53 \\
\hline UiO-66 & $\mathrm{Zr} / \mathrm{BDC}$ & PVA, 9.3 & - & 1378 & $1195(-14 \%)$ & 54 \\
\hline UiO-66- $\mathrm{NH}_{2}$ & $\mathrm{Zr} / \mathrm{NH}_{2}-\mathrm{BDC}$ & None & 127 & 1182 & $876(-26 \%)$ & 51 \\
\hline UiO-66- $\mathrm{NH}_{2}$ & $\mathrm{Zr} / \mathrm{NH}_{2}-\mathrm{BDC}$ & None & 164 & 839 & $625(-26 \%)$ & 49 \\
\hline UiO-66-COOH & $\mathrm{Zr} / \mathrm{BDC}-\mathrm{COOH}$ & None & 9.7 & 710 & $614(-14 \%)$ & 55 \\
\hline UiO-67 & $\mathrm{Zr} / \mathrm{BPDC}$ & None & 63 & 2034 & $1549(-24 \%)$ & 49 \\
\hline MOF-801 & $\mathrm{Zr} / \mathrm{FA}$ & PVB, 5 & 146 & 899 & $569(-37 \%)$ & 58 \\
\hline
\end{tabular}

Linker codes: BDC - benzene-1,4-dicarboxylic acid; BTB - 1,3,5-benzenetribenzoate; MIM - 2-methyl imidazole; MIC - 4-methyl-5imidazolecarboxaldehyde; BTC - benzene-1,3,5-tricarboxylic acid; DHBDC - 2,5-dihydroxy-1,4-benzenedicarboxylic acid; BPDC - biphenyl-4,4'dicarboxylic acid; and FA - formic acid. Binder codes: PVA - polyvinyl alcohol; SB - pseudoboehmite; and PVB - polyvinyl butyral. "- " not specified. ${ }^{a}$ Used as an additive to improve thermal conductivity.

properties. Additionally, the rate of pressure increase should be adequate for the same reason.

The primary goal of pelletization is the formation of densely packed pellets for further employment in large fixed-bed type reactors, or transportation applications in the specific case of MOFs. Many industrial processes rely on using such pellets as adsorbents and catalysts. Indeed, it is easier to handle shaped objects than their powder analogues. Besides, they minimize the risk of a pressure drop, afford an enhanced mixing and distribution inside the bed volume and therefore allow for more efficient production processes. These are achieved upon providing the pellets with pronounced resistance to attrition and breakage.

Compaction itself serves as a source of reinforcement; however, sometimes the use of binders to enhance the mechanical stability of pellets is of particular interest. Binders are usually classified into organic binders such as starch, cellulose and polyvinyl alcohol (PVA) and inorganic binders such as clays, silica and graphite. ${ }^{20}$ They facilitate bonding of individual particles by generating a link between them. As an example, it was shown that zeolites $\mathrm{X}$ and $\mathrm{Y}$ could be pelletized using bentonite as the binder, ${ }^{21}$ and kaolinite could be employed to bind ZSM-5 zeolite crystals together. ${ }^{22}$ In both cases there is an alteration of both the physical and chemical properties of the final materials compared to the pristine zeolites.

Generally, pelletization leads to drastic losses of textural properties when applied to highly-porous materials. MOFs are especially sensitive to these losses as their frameworks are more fragile and flexible than frameworks composed of inorganic building units. This becomes even more pronounced when pelletization is performed with a binder. Although protocols found for pelletization can largely differ in the literature, Table 3 illustrates the effect of pelletization on MOFs depending on the metal oxidation state and associated linker. 
As is evident from the results presented in Table 3 , the pelletization process leads to drastic decreases of textural properties as evaluated by the specific surface area, and especially in the case of metals with low coordination $(\mathrm{Cu}, \mathrm{Zn})$ - with the notable exception of ZIF-8. High applied pressure reduces interparticular voids and can even collapse the frameworks, while addition of binders leads to pore blocking. One of the earliest studies on pelletization of MOFs was performed by Finsy et al. ${ }^{23}$ who studied $\mathrm{CO}_{2} / \mathrm{CH}_{4}$ adsorption on Al-based MIL53 pellets. Particularly, in their work the MOF was mixed with the binder, an aqueous solution of polyvinyl alcohol, and upon homogenization heated at a rate of $0.5{ }^{\circ} \mathrm{C} \mathrm{min}{ }^{-1}$ up to $190{ }^{\circ} \mathrm{C}$. Such a procedure yielded pellets with a binder content of $13 \mathrm{wt} \%$, which were then used for $\mathrm{CO}_{2} / \mathrm{CH}_{4}$ mixture adsorption. Although no pressure was applied to form pellets, the total accessible pore volume decreased by $19 \mathrm{wt} \%$, which exceeds the content of PVA and suggests partial pore blocking. Following this, the authors further studied the binderless pelletization of V-based MIL-47 to separate xylene isomers ${ }^{24}$.

\section{MOF-5}

Among other early studies, there is a study by Alcañiz-Monge et $a .^{25}$ addressing the effects of compression on the textural properties of MOF-5 compared to silica and zeolites. Typically, each solid was compressed in a $1.45 \mathrm{~cm}$ diameter die up to 420 MPa. As expected, the specific surface areas and pore volumes decreased when the applied pressure increased. Predictably, inorganic solids exhibiting frameworks with high mechanical strength were less affected by compression and therefore less prone to drastic losses in surface area. In contrast, MOF-5 whose framework is formed by coordination bonds has lower mechanical strength and is therefore heavily affected by compression. Indeed, its compression at $180 \mathrm{MPa}$ led to a severe broadening of reflections in the XRD pattern, suggesting structural collapse of the framework. This was supported by $\mathrm{N}_{2}$ physisorption measurements which yielded an extremely low surface area of $275 \mathrm{~m}^{2} \mathrm{~g}^{-1}$ for the pellet as compared to 2160 $\mathrm{m}^{2} \mathrm{~g}^{-1}$ for the parent powder. Considering these observations, this allows setting up an upper limit for compression forces applied to MOF-5 to $180 \mathrm{MPa}$, at which this MOF experiences quasi-total structural collapse.

In another early study on MOF-5, Hu and $\mathrm{Zhang}^{26}$ demonstrated its irreversible amorphization starting from 3.5 MPa. Indeed, the authors showed evidence of peak broadening in the XRD patterns as the compression pressure increased. Moreover, Raman spectroscopy revealed that the MOF-5 structure collapsed via the breaking of the bond between $\mathrm{Zn}$-clusters and the BDC linker. Therefore, upon compression up to 10.3 MPa the final BET surface area was only $6 \mathrm{~m}^{2} \mathrm{~g}^{-1}$ as compared to $3450 \mathrm{~m}^{2} \mathrm{~g}^{-1}$ of the pristine powder. Further studies on MOF-5 pelletization include the study by Jia et al. ${ }^{27}$ who used the MOF for methane adsorption at ambient temperature. The results suggest that a mild compression pressure of up to $2 \mathrm{MPa}$ applied to the MOF-5 powder led to a decrease of the BET surface area from 2777 to $1450 \mathrm{~m}^{2} \mathrm{~g}^{-1}$. The MOF-5 pellet retained around $50 \%$ of its initial surface area; however, volumetric methane uptake measurements revealed lower values when compared to the initial powder. Thus, the MOF-5 volumetric methane capacity was stated to be overestimated. Therefore, later studies on MOF-5 mainly aimed at hydrogen storage. Purewall et al. ${ }^{28}$ conducted a study on MOF-5 densification to improve its volumetric hydrogen uptake. Typically, they evaluated the performance of the MOF upon manual pressing until the desired density was reached. Noteworthily, they observed the preservation of the crystal structure and therefore no transformation into an amorphous phase even when pressing the powder until a bulk density of $0.75 \mathrm{~g} \mathrm{~cm}^{-3}$ was reached, which corresponded to approximately $80 \mathrm{MPa}$ applied compression pressure. This is in agreement with earlier studies $^{25}$ and differs from the one obtained by $\mathrm{Hu}$ and Zhang. ${ }^{26}$ Nevertheless, the specific surface area decreased accordingly with increasing pressure, from $2762 \mathrm{~m}^{2} \mathrm{~g}^{-1}$ for the pristine powder to $1188 \mathrm{~m}^{2} \mathrm{~g}^{-1}$ for the pellet with a bulk density of $0.90 \mathrm{~g} \mathrm{~cm}^{-3}$. In agreement with that, the hydrogen adsorption capacity varied accordingly. Thus, a densified pellet of $0.51 \mathrm{~g} \mathrm{~cm}^{-3}$ demonstrated a 3.5 times increase in excess volumetric hydrogen uptake, reaching $26 \mathrm{~g} \mathrm{~L}^{-1}$, with only a $15 \%$ decrease in gravimetric uptake at $-196{ }^{\circ} \mathrm{C}$ as compared to its powder counterpart. Additionally, the authors performed a complementary study ${ }^{29,30}$ to address the enhancement of thermal conductivity of the densified MOF-5 pellets, an important parameter defining the hydrogen refueling rate and therefore the material's efficiency. Adding $10 \mathrm{wt} \%$ expanded natural graphite (ENG) as an additive (Fig. 2a) increased the thermal conductivity of the final MOF-5/ENG composites by a factor of 5 while decreasing the volumetric hydrogen uptake only by $6 \%$ as compared to the neat MOF-5 pellet of $0.51 \mathrm{~g} \mathrm{~cm}^{-3}$. Interestingly, they further showed that pelletization of MOF-5 (Fig. 2b and c) improved its stability against water as it slowed down subsequent degradation under moderately humid conditions. ${ }^{31}$

\section{MOF-177}

Zacharia et al. ${ }^{32}$ studied the volumetric hydrogen uptake capacities of MOF-177 pellets prepared by compression. It is worth mentioning that this $\mathrm{Zn}$-based MOF is commercially available, making it interesting for large-scale applications. The authors applied various pressures to prepare monoliths with different densities. It was found that above $0.51 \mathrm{~g} \mathrm{~cm}^{-3}$ MOF-177 underwent amorphization as the XRD patterns of MOF-177 upon compression exhibited broad reflections. Moreover, its BET surface area decreased accordingly from around $4100 \mathrm{~m}^{2} \mathrm{~g}^{-1}$ for the pristine powder to 3300 and $2400 \mathrm{~m}^{2} \mathrm{~g}^{-1}$ for pellets compressed to bulk densities of 0.51 and $0.59 \mathrm{~g} \mathrm{~cm}^{-3}$, respectively. Noteworthily, when assessed for hydrogen adsorption at $-196{ }^{\circ} \mathrm{C}$ and $\sim 8 \mathrm{MPa}$, the total $\mathrm{H}_{2}$ uptake reached $41 \mathrm{~g} \mathrm{~L}^{-1}$ over the MOF-177 pellets with a bulk density of $0.53 \mathrm{~g} \mathrm{~cm}^{-3}$. Under the same conditions the total $\mathrm{H}_{2}$ uptake reached $41 \mathrm{~g} \mathrm{~L}^{-1}$ at $8 \mathrm{MPa}$ on MOF-5 pellets with a bulk density of $0.51 \mathrm{~g} \mathrm{~cm}^{-3} .^{28}$ Dailly and Poirier ${ }^{33}$ further evaluated the hydrogen uptake on MOF-177 pellets provided by BASF over a wide range of temperatures. The results suggest that at $-196{ }^{\circ} \mathrm{C}$ and $4 \mathrm{MPa}$, MOF177 pellets exhibited around $36 \mathrm{~g} \mathrm{~L}^{-1}$ total $\mathrm{H}_{2}$ volumetric 

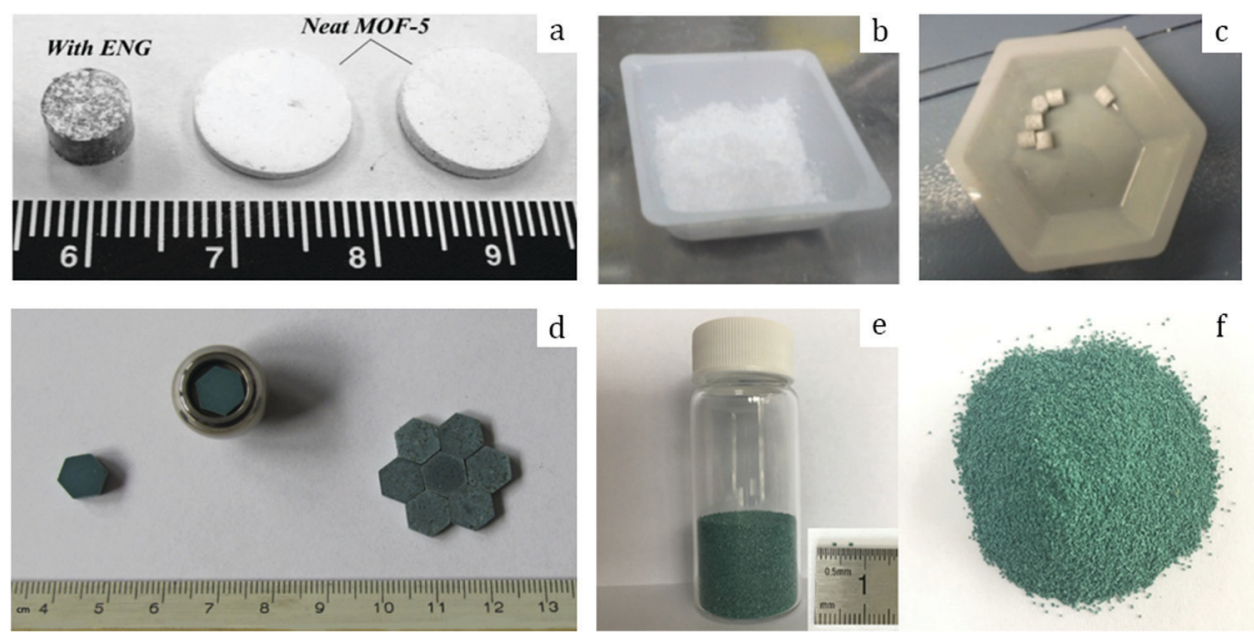

Fig. 2 Pure MOF-5 pellets and MOF-5/ENG (expanded natural graphite) composites (a) made by Liu et al. ${ }^{30}$ Copyright 2012 Elsevier. MOF-5 powder (b) and pellets (c) prepared by Ming et al. ${ }^{31}$ Copyright 2015 American Chemical Society. Hexagonal MIL-101 monoliths (d) prepared by Blanita et al. ${ }^{43}$ Copyright 2014 Elsevier. MIL-100 granules (e and f) with an average size of around $1 \mathrm{~mm}$ made from compressed pellets by Yang et al. ${ }^{56}$ Copyright 2019 American Chemical Society.

uptake. It is in a good agreement with the values reported by Purewall $e t a .^{28}$ and considerably higher than that of the pristine powder, $26 \mathrm{~g} \mathrm{~L}^{-1}$ reported by Zacharia et $a .^{32}$

\section{MIL-53}

Al-based MIL-53 is also among the commercially available MOF structures, and is therefore readily available for gas studies. Thus, Heymans et al. ${ }^{34}$ applied the densified MIL-53 supplied by BASF for gas separation. Namely, they used it for the separation of $\mathrm{CO}_{2}$ and $\mathrm{H}_{2} \mathrm{~S}$ from a stream of $\mathrm{CH}_{4}$. The results demonstrated preferential adsorption of $\mathrm{H}_{2} \mathrm{~S}$ over $\mathrm{CO}_{2}$ and $\mathrm{CH}_{4}$ at $30{ }^{\circ} \mathrm{C}$ over a range of gas pressures. At the same time, it was shown that MIL-53 in pellet form adsorbed less $\mathrm{H}_{2} \mathrm{~S}$ as compared to its powder counterpart ( 8 vs. $10 \mathrm{~mol} \mathrm{~kg}^{-1}$ ) - a regular phenomenon upon densification. Noteworthily, the total $\mathrm{CO}_{2}$ uptake decrease upon densification was consistent with the decrease in pore volume and was nearly $20 \%$. Besides, Moreira et al. ${ }^{35}$ showed that MIL-53 could also be used as an adsorbent for liquid phase separations. This MOF exhibited preferential adsorption of $o$-xylene and therefore a certain capacity of separation from a mixture with $p$ - and $m$-isomers. The work of Majchrzak-Kuceba and Sciubidlo ${ }^{36}$ addressed the $\mathrm{CO}_{2}$ adsorption by densified MIL-53. For this purpose, they applied different compression pressures on the MOF powder. It was shown that MIL-53 pellets could be used as an adsorptive bed for $\mathrm{CO}_{2}$ removal; however, their XRD patterns (Fig. 3 in the original article) suggest major amorphization of the MIL-53 framework upon pelletization at $29.6 \mathrm{kN} \mathrm{m}^{-2}$. MIL-53 is famous for its "breathing behavior", owing to its flexible structure. Interestingly, a structural transition from large pores (MIL-53lp) to narrow pores (MIL-53-np) was observed in the compression range from 3.7 to $7.4 \mathrm{kN} \mathrm{m}^{-2}$.

The impact of compression on the textural and crystalline properties of MIL-53, as well as on its $\mathrm{CO}_{2}$ sorption properties, was also studied by Ribeiro et al. ${ }^{37}$ They compressed MIL-53 at
62 and $125 \mathrm{MPa}$ with no binder added. The crystallinity was preserved even upon densification at $125 \mathrm{MPa}$ as the XRD patterns were identical to their original powder counterpart. There is, however, a small shift of the reflections towards larger $2 \theta$ values. The authors attributed this phenomenon to the structural deformations of the framework upon compression, in accordance with the results provided by Majchrzak-Kuceba and Sciubidlo. ${ }^{36}$ In addition, it was shown that the textural properties were altered accordingly. Thus, MIL-53 lost 46 and $32 \%$ of the available surface as well as 36 and $24 \%$ of the pore volume, which can be partly attributed to the transition from a system of large pores to narrow pores, upon densification at 125 and $62 \mathrm{MPa}$, respectively. Interestingly, a broad distribution of macropores at around $230 \mathrm{~nm}$ was observed. Furthermore, the $\mathrm{CO}_{2}$ adsorption capacity of MIL-53 decreased from $5.2 \mathrm{~mol} \mathrm{~kg}^{-1}$ to 3.7 and $4.0 \mathrm{~mol} \mathrm{~kg}^{-1}$ when compressed at 1.5 , 62 , and $125 \mathrm{MPa}$, respectively. The reduced gas uptake is thus consistent with the decrease in pore volume. Therefore, pelletization is considered as an appropriate shaping method for this MOF.

\section{ZIF-8}

Ribeiro et $a l .{ }^{37}$ also extended their study to ZIF-8. As in the case of MIL-53, the ZIF-8 crystallinity was preserved even upon densification at $125 \mathrm{MPa}$ as the XRD patterns matched with the original powder counterpart. In addition, it was shown that its textural properties were altered accordingly. Thus, ZIF-8 experienced only 7 and $12 \%$ loss in BET surface area as well as in pore volume when compressed at 62 and $125 \mathrm{MPa}$, respectively. Interestingly, it was demonstrated that densification led to the creation of macropores with diameters of about $300 \mathrm{~nm}$ and a distribution narrower than in the case of MIL-53. Furthermore, the $\mathrm{CO}_{2}$ adsorption capacity decreased with the pore volume, from $9.0 \mathrm{~mol} \mathrm{~kg}^{-1}$ with the initial powder, to $8.7(-3 \%)$ 
Table 4 Textural and mechanical properties of ZIF-8 pellets compressed with 10 wt\% SB and different solvents (10 wt\%): NA - nitric acid, CA - citric acid, TA - trichloroacetic acid ${ }^{40}$

\begin{tabular}{lrllllll}
\hline & & & \multicolumn{5}{c}{$\begin{array}{l}\text { Gas adsorption capacities } \\
\text { at } 25{ }^{\circ} \mathrm{C}, \mathrm{mg} \mathrm{g}^{-1}\end{array}$} \\
\cline { 5 - 8 } Pellet & \multicolumn{1}{c}{$S_{\mathrm{BET}}}$, & $\begin{array}{l}\text { Mechanical } \\
\text { strength, MPa }\end{array}$ & $\mathrm{CO}_{2}$ & $\mathrm{CH}_{4}$ & $\mathrm{C}_{3} \mathrm{H}_{8}$ & $\mathrm{C}_{3} \mathrm{H}_{6}$ \\
\hline ZIF-8 & 1023 & $0.19(=)$ & 340 & 100 & 183 & 210 \\
ZIF-8/SB/NA & 959 & $1.71(+811 \%)$ & 270 & 81 & 158 & 170 \\
ZIF-8/SB/CA & 933 & $1.59(+748 \%)$ & 280 & 84 & 172 & 190 \\
ZIF-8/SB/TA & 1161 & $0.75(+300 \%)$ & 310 & 92 & 175 & 195 \\
\hline
\end{tabular}

and $8.5 \mathrm{~mol} \mathrm{~kg}^{-1}(-6 \%)$ with pellets pressed at 62 and $125 \mathrm{MPa}$, respectively.

Chapman et $a .^{38}$ studied ZIF-8 compression at industrially relevant pressures up to $1000 \mathrm{MPa}$. They reported an irreversible structural transformation (amorphization) at pressures higher than $340 \mathrm{MPa}$. This sets an upper limit for ZIF-8 compression. Interestingly, the authors observed that upon amorphization the ZIF-8 framework remained porous, however with modified sorption behavior. Thus, upon compressing at 300 MPa ZIF-8 lost $\sim 13 \%$ of its initial available surface area.

Among other studies on ZIF-8 densification, there is a study by Bazer-Bachi et $a l .{ }^{39}$ (who also densified SIM-1). The authors applied a wide range of pressures and showed that the crystallinity of ZIF-8 was preserved upon compression up to $\sim 230$ MPa. At the same time, the loss in BET surface area was about $11 \%$, with the ZIF-8 pellet reaching $1278 \mathrm{~m}^{2} \mathrm{~g}^{-1}$, while the pristine ZIF-8 powder exhibited $1433 \mathrm{~m}^{2} \mathrm{~g}^{-1}$. Noteworthily, these results are in good agreement with the ones reported by Ribeiro et al. ${ }^{37}$ and Chapman et al. ${ }^{38}$ Upon compression, SIM-1 demonstrated a similar trend with a $28 \%$ drop in surface area (516 vs. $370 \mathrm{~m}^{2} \mathrm{~g}^{-1}$ ) at a decent pressure of $\sim 400 \mathrm{MPa}$ while preserving its framework topology according to its XRD pattern.

The study by Zhu et al. ${ }^{40}$ concerned the effect of the type of binder on the textural and adsorption properties of ZIF-8 upon compression. More precisely, addition of $10 \mathrm{wt} \% \mathrm{SB}$ (high quality pseudoboehmite) or talc powder led to a slightly reduced BET surface area as compared to the pristine ZIF- 8 powder. In contrast, addition of $10 \mathrm{wt} \%$ bentonite or methylcellulose led to a more severe decrease in surface area. Besides, the authors studied the influence of acidic solvents on the final properties of the compressed pellets. Thus, mixing the initial ZIF-8/binder blend with $10 \mathrm{wt} \%$ citric acid led to a larger decrease in surface area as compared to other probed solvents such as nitric and trichloroacetic acid. Out of the three acids, nitric acid demonstrated the best compromise between high mechanical strength provided to the shaped pellets and high $S_{\text {BET }}$ (Table 4).

As is evident from Table 4, although the textural and adsorption properties of the pellets were lower when ZIF-8 was compressed in the presence of an acid, the resulting objects exhibited superior mechanical properties. This might be attributed to the acidic strength of the solvents and therefore their ability to stick individual MOF and SB particles. Indeed, nitric acid is the strongest among the three and hence provided better peptization of SB particles. Besides, the authors found that the adsorption capacities of the pellets were not altered as the loss upon shaping is consistent with the amount of the binder added.

\section{MIL-101(Cr)}

Densification of Cr-based MIL-101 for hydrogen adsorption was extensively studied by Ardelean et al. ${ }^{41}$ The parent MOF powder was compressed into pellets with controlled bulk density up to a maximum value of $1.34 \mathrm{~g} \mathrm{~cm}^{-3}$. The authors found that the MIL-101 crystal structure was preserved at intermediate pellet bulk densities of up to $0.47 \mathrm{~g} \mathrm{~cm}^{-3}$, while the XRD pattern indicated transformation into an amorphous phase when compressed at $1.34 \mathrm{~g} \mathrm{~cm}^{-3}$. The specific surface area decreased accordingly, down to $2602 \mathrm{~m}^{2} \mathrm{~g}^{-1}$ for the pellets with a bulk density of $0.47 \mathrm{~g} \mathrm{~cm}^{-3}$ as compared to $3623 \mathrm{~m}^{2} \mathrm{~g}^{-1}$ for the MIL101 powder. Nevertheless, when the total volumetric hydrogen capacity was assessed, pellets with densities in the range of $0.45-0.47 \mathrm{~g} \mathrm{~cm}^{-3}$ exhibited $40 \mathrm{~g} \mathrm{~L}^{-1} \mathrm{H}_{2}$ uptake at $-196^{\circ} \mathrm{C}$ and 8 MPa. This value is in a good agreement with the previously reported values for MOF- $177^{32}$ and MOF- $5 .^{28}$ Following this, Oh et al. ${ }^{42}$ focused on setting an upper limit of $\mathrm{H}_{2}$ uptake capacity for MIL-101 pellets which was found to be $42.3 \mathrm{~g} \mathrm{~L}^{-1}$ at $-253{ }^{\circ} \mathrm{C}$. In further studies, Blanita et al. ${ }^{43}$ studied the $\mathrm{H}_{2}$ adsorption capacity of close-packed hexagonal MIL-101 pellets (Fig. 2d). The total volumetric $\mathrm{H}_{2}$ uptake reached comparable $40.3 \mathrm{~g} \mathrm{~L}^{-1}$ at $-196{ }^{\circ} \mathrm{C}$ and $10 \mathrm{MPa}$ for the pellets with a bulk density of $0.47 \mathrm{~g} \mathrm{~cm}^{-3}$. At $15 \mathrm{MPa}$, the capacity further increased to $43.7 \mathrm{~g} \mathrm{~L}^{-1}$. Finally, the authors studied the effect of Al tape addition in order to increase the thermal conductivity between MIL-101 pellets and therefore to improve the kinetics of $\mathrm{H}_{2}$ adsorption/desorption cycles. ${ }^{44}$ Indeed, 8-9 wt\% Al tapes led to an increase of the thermal conductivity, up to $\sim 0.5 \mathrm{~W} \mathrm{~m}^{-1} \mathrm{~K}^{-1}$, similar to the values reported by Purewall et al. ${ }^{29}$ In turn, such MOF composites presented enhanced $\mathrm{H}_{2}$ adsorption/desorption rates, allowing $\sim 0.9$ fractional uptake/ release in around $40 \mathrm{~s}$.

\section{MOF-74(Ni), (Mg)}

This family of MOFs is also known as CPO-27. Tagliabue et al. ${ }^{45}$ studied the effect of compression on the textural and adsorption properties of Ni-based CPO-27. They found that the MOF preserved its crystal structure when compressed at $100 \mathrm{MPa}$, while it collapsed at $1000 \mathrm{MPa}$. This was supported by $\mathrm{N}_{2}$ physisorption measurements which confirmed the microporous nature of the former pellet with a BET surface area of $1050 \mathrm{~m}^{2} \mathrm{~g}^{-1}$ - as compared to $1060 \mathrm{~m}^{2} \mathrm{~g}^{-1}$ for the pristine CPO27 powder. At the same time, the pellet compressed at $1000 \mathrm{MPa}$ showed negligible BET surface area. Besides, it was demonstrated that the $\mathrm{CH}_{4}$ adsorption on the shaped MOF was $129 \mathrm{~N} \mathrm{~cm}^{3} \mathrm{~g}^{-1}$ at $30{ }^{\circ} \mathrm{C}$ and $3.4 \mathrm{MPa}$, as opposed to $157 \mathrm{~N} \mathrm{~cm}^{3} \mathrm{~g}^{-1}$ for the parent powder MOF, indicating an $18 \%$ loss in gravimetric capacity upon compression.

Remy et $a l .{ }^{46}$ studied the adsorption properties of another MOF with the CPO-27/MOF-74 topology (Mg-MOF-74) upon pelletization. First, they found that addition of a few drops of methanol into the parent powder preserved the MOF crystal 
structure upon pressing at $30 \mathrm{MPa}$ for 1 minute. The thusshaped pellets demonstrated a BET surface area of $1034 \mathrm{~m}^{2} \mathrm{~g}^{-1}$, lower than that of the parent powder $\left(1249 \mathrm{~m}^{2} \mathrm{~g}^{-1}\right)$. However, the pellets exhibited a similar $\mathrm{CO}_{2}$ adsorption capacity at $0.05 \mathrm{MPa}$ and $30{ }^{\circ} \mathrm{C}$ to the parent $\mathrm{Mg}-\mathrm{MOF}-74$ powder, i.e. 7.3 and $7.8 \mathrm{mmol} \mathrm{g}^{-1}$, respectively. Moreover, they show strong affinity for $\mathrm{CO}_{2}$ and therefore successfully separated $\mathrm{CO}_{2}$ from an equimolar $\mathrm{CO}_{2} / \mathrm{CH}_{4}$ mixture. Interestingly, they found that Mg-MOF-74 pellets outperformed zeolite 13X, a benchmark adsorbent, for the $\mathrm{CO}_{2}$ adsorption at $0.1 \mathrm{MPa}$ and $35{ }^{\circ} \mathrm{C}$ on a gravimetric basis (6.9 vs. $4.8 \mathrm{mmol} \mathrm{g}^{-1}$ ). However, the latter performed better on a volumetric basis ( $\left.6.9 \mathrm{vs} .6 .0 \mathrm{kmol} \mathrm{m}^{-3}\right)$. Nevertheless, the MOF pellets showed an easier regeneration than the zeolite. Importantly, the authors outlined the lower stability of Mg-MOF-74 pellets against impurities such as $\mathrm{O}_{2}$ and $\mathrm{H}_{2} \mathrm{O}$ over a series of runs as compared to zeolite $13 \mathrm{X}$, making the latter the preferred adsorbent for low-pressure $\mathrm{CO}_{2}$ separation.

\section{HKUST-1}

Alcañiz-Monge et $a l^{25}$ conducted a study on the pelletization of Cu-based HKUST-1 powder, presenting an initial $S_{\text {BET }}$ of $1145 \mathrm{~m}^{2} \mathrm{~g}^{-1}$. However, when compressed at $180 \mathrm{MPa}$, the resulting HKUST-1 pellets exhibited a halved SSA, i.e. $470 \mathrm{~m}^{2} \mathrm{~g}^{-1}$. A further increase of the pressure up to $420 \mathrm{MPa}$ had no further significant impact on SSA $\left(420 \mathrm{~m}^{2} \mathrm{~g}^{-1}\right)$. Consequently, the XRD patterns recorded for this MOF yielded no reflections, implying structural disorder. Considering these observations, this enables setting up an upper limit for compression force for HKUST-1 at $180 \mathrm{MPa}$. Above this value, the framework experiences structural collapse.

Peterson et ll. $^{47}$ performed another study on HKUST-1 to examine the evolution of its physical and chemical properties. Thus, the authors applied pressures of $1000 \mathrm{psi}(\sim 7 \mathrm{MPa})$ and 10000 psi ( $\sim 69 \mathrm{MPa})$. While the crystal structure was globally preserved, compressed HKUST-1 exhibited broader reflections as well as high signal-to-noise ratios on the XRD patterns. This suggests partial framework damage. Consequently, there was a certain decrease in BET surface area, from $1698 \mathrm{~m}^{2} \mathrm{~g}^{-1}$ for the powder to $892 \mathrm{~m}^{2} \mathrm{~g}^{-1}$ for the pellets made at $\sim 69 \mathrm{MPa}$. These values are somewhat different from the ones reported by Kim et al., ${ }^{48}$ who stated that above $10 \mathrm{MPa}$ the HKUST-1 framework underwent structural degradation. At the same time, Dhainaut et al. ${ }^{49}$ reported a low (15\%) loss in BET surface area for HKUST-1, reaching $1091 \mathrm{~m}^{2} \mathrm{~g}^{-1}$ upon densification at $121 \mathrm{MPa}$. Besides, they showed that addition of $2 \mathrm{wt} \%$ of a binder (graphite) slightly improved the mechanical stability of HKUST-1 pellets without significant loss of BET surface area. They explained this relatively small loss as due to the presence of the remaining solvent within the framework, acting as a scaffold during compression, as well as the slow compression speed applied to the powder bed.

Interestingly, in a study by Peterson et $a l .{ }^{47}$ the $\mathrm{N}_{2}$ physisorption isotherms exhibit a hysteresis, suggesting the creation of mesopores upon pelletization due to the breakage of framework bonds. Besides, when measuring $\mathrm{NH}_{3}$ breakthrough curves, they demonstrated that ammonia eluted faster through the pelletized HKUST-1 as compared to the pure powder owing to its faster diffusion through the mesopores. Importantly, the total $\mathrm{NH}_{3}$ loading remained intact around $7.2 \mathrm{mmol} \mathrm{g}^{-1}$ for all materials, indicating that the sorption sites in HKUST-1 remain available upon compression up to $69 \mathrm{MPa}$.

Bazer-Bachi et $a .^{39}$ also addressed the impact of pressure applied on HKUST-1. Consequently, it was clearly more affected by compression than two other MOFs tested (ZIF-8 and SIM-1). Indeed, already at an applied pressure of $\sim 24 \mathrm{MPa}$, HKUST-1 lost around $27 \%$ of its initial available surface area (1897 vs. $1377 \mathrm{~m}^{2} \mathrm{~g}^{-1}$ ). Moreover, at a higher pressure of $\sim 400 \mathrm{MPa}$, the surface area further decreased by $76 \%$, accounting for $453 \mathrm{~m}^{2} \mathrm{~g}^{-1}$, while its XRD pattern displayed broad reflections with poor intensities, suggesting partial collapse of the framework.

Terracina et al. $^{50}$ outlined the crucial role of thermal activation in pelletization of HKUST-1. They found that the structural integrity and textural properties could be preserved to a great extent upon activation of the powder right before tableting and reactivation at $147^{\circ} \mathrm{C}$ for $1 \mathrm{~h}$ immediately after compression. By doing so, the authors demonstrated that the damaging effect of coordinated water molecules could be decreased, as suggested by EPR measurements. Besides, the resulting pellets presented a higher BET surface area of $1935 \mathrm{~m}^{2} \mathrm{~g}^{-1}$ as compared to $1620 \mathrm{~m}^{2} \mathrm{~g}^{-1}$ for the as-made powder. The higher BET surface area could be due to the creation of additional mesopores, as underlined by Peterson et al. ${ }^{47}$ Within the range of applied pressures, the authors found that $40 \mathrm{MPa}$ is an optimal value to compress HKUST-1 into pellets with a good compaction degree.

\section{UiO-66 family}

Peterson et al. ${ }^{51}$ performed a study on the compression of $\mathrm{Zr}$ based UiO-66- $\mathrm{NH}_{2}$. They applied a range of pressures from 5000 to $100000 \mathrm{psi}(\sim 35$ to $690 \mathrm{MPa})$ and observed only minor changes in textural properties upon compressing the powder up to $25000 \mathrm{psi}(\sim 172 \mathrm{MPa})$. At this value, they found that both the crystallinity and structural integrity remained intact. At the same time, the BET surface area decreased from 1182 to $876 \mathrm{~m}^{2} \mathrm{~g}^{-1}$. Besides, upon compression the UiO-66- $\mathrm{NH}_{2}$ pellets mostly retained their efficiency for toxic gas removal (Table 5). Noteworthily, compressed pellets exhibited shorter breakthrough times than their powder analogues especially in the case of $\mathrm{NH}_{3}$, suggesting diffusion limitations or lower adsorption capacity. The latter is in line with the lower SSA measured.

Under the same conditions, the UiO-66 framework proved to be more stable toward high pressures. ${ }^{47}$ Upon compression up

Table $5 \mathrm{NH}_{3}$ and $\mathrm{CNCl}$ adsorption capacities of $\mathrm{UiO}-66-\mathrm{NH}_{2}$ in both powder and pellet forms ${ }^{51}$

\begin{tabular}{|c|c|c|c|c|}
\hline \multirow[b]{2}{*}{ Adsorbent } & \multirow{2}{*}{$\begin{array}{l}\text { Applied } \\
\text { pressure, } \\
\text { MPa }\end{array}$} & \multirow{2}{*}{$\begin{array}{l}\text { BET } \\
\text { surface area, } \\
\mathrm{m}^{2} \mathrm{~g}^{-1}\end{array}$} & \multicolumn{2}{|c|}{$\begin{array}{l}\text { Gas loadings under } \\
\text { dry (humid) conditions, } \\
\text { mol kg }{ }^{-1}\end{array}$} \\
\hline & & & $\mathrm{NH}_{3}$ & $\mathrm{CNCl}$ \\
\hline UiO-66- $\mathrm{NH}_{2}$-powder & - & 1182 & $3.3(2.9)$ & $4.1(1.2)$ \\
\hline UiO-66- $\mathrm{NH}_{2}$-pellet & 172 & 876 & $2.2(1.8)$ & $2.7(0.9)$ \\
\hline
\end{tabular}


to $69 \mathrm{MPa}$, the BET surface area of the pellet reached $1080 \mathrm{~m}^{2} \mathrm{~g}^{-1}$, which is identical to that of the parent powder. Therefore, when tested for octane adsorption, the UiO-66 pellet compressed at $\sim 69 \mathrm{MPa}$ demonstrated a saturation loading comparable to its powder counterpart (2.1 vs. $2.5 \mathrm{mmol} \mathrm{g}^{-1}$, respectively).

Moreira et $a l .{ }^{52}$ demonstrated the reverse selectivity of UiO66 towards liquid-phase separation of xylene isomers. Indeed, the obtained results suggested $o$-xylene selectivities of 1.8 and 2.4 with respect to $m$ - and $p$-xylene, at $40{ }^{\circ} \mathrm{C}$ with $n$-heptane as the eluent. Besides, the authors showed that the selectivities were retained upon compression, meaning that no major modification of the pore network took place upon compression. Interestingly, the authors stated that at low concentrations the selectivity values of UiO-66 were comparable to the ones previously reported for MIL-53. However, the latter failed to separate $m$ - and $p$-isomers unlike UiO-66.

Dhainaut et $a l .{ }^{49}$ reported a detailed study of the effect of compression on the textural properties of some of the most studied MOFs including UiO-66, UiO-66- $\mathrm{NH}_{2}$ and UiO-67. They found that the impact of pressing UiO-66 and UiO-66- $\mathrm{NH}_{2}$ was in line with the pressure applied as their textural properties decreased accordingly. Thus, they reported a $26 \%$ decrease in UiO-66- $\mathrm{NH}_{2}$ BET surface area upon compression at $164 \mathrm{MPa}$, which is in good agreement with the results reported by Peterson et al. ${ }^{51}$ Interestingly, it was found that the UiO-67 structure started collapsing upon compression above $63 \mathrm{MPa}$, while at $82 \mathrm{MPa}$ it lost $\sim 80 \%$ of its initial surface area (2034 vs. $397 \mathrm{~m}^{2} \mathrm{~g}^{-1}$ ). Based on their results, they proposed to limit the compression to a final bulk density that represents at most $80 \%$ of the crystal density of the related MOF.

Bambalaza et al. ${ }^{53}$ showed the possibility to press UiO-66 at even higher pressures to improve its volumetric $\mathrm{H}_{2}$ uptake. They applied a wide range of pressures: 150, 290, 440, 590 and $665 \mathrm{MPa}$ to study how the textural and structural properties would be modified. Consequently, they found that even at the highest compression pressure, $665 \mathrm{MPa}$, the framework retained its crystal structure. Besides, its BET surface area only decreased by $\sim 5 \%$ from $1737 \mathrm{~m}^{2} \mathrm{~g}^{-1}$ for the powder to $1707 \mathrm{~m}^{2} \mathrm{~g}^{-1}$ for the pellet. However, the loss of total pore volume was more significant and reached $16 \%$ (from 0.96 to $\left.0.81 \mathrm{~cm}^{3} \mathrm{~g}^{-1}\right)$. Importantly, the thus-formed pellets demonstrated a remarkable increase of volumetric $\mathrm{H}_{2}$ uptake, from 29 to $74 \mathrm{~g} \mathrm{~L}^{-1}$ at $-196{ }^{\circ} \mathrm{C}$ and $10 \mathrm{MPa}$ as well as from 6 to $13 \mathrm{~g} \mathrm{~L}^{-1}$ when measured at $25{ }^{\circ} \mathrm{C}$ and $10 \mathrm{MPa}$. These results suggested a positive effect of compression on the packing density of UiO-66 $\left(1.45 \mathrm{~g} \mathrm{~cm}^{-3}\right.$ at $\left.665 \mathrm{MPa}\right)$ and therefore its benefits for improved volumetric $\mathrm{H}_{2}$ uptake without a significant reduction of the gravimetric $\mathrm{H}_{2}$ uptake.

Edubilli and Gumma ${ }^{54}$ studied UiO-66 pelletization with addition of a PVA aqueous solution, aiming at a binder content of $9.3 \mathrm{wt} \%$ in the final dried pellets. They showed that this composition was optimal to provide enough mechanical strength to withstand a drop test. However, the presence of PVA caused slight pore blocking and it resulted in a decreased BET surface area, from $1378 \mathrm{~m}^{2} \mathrm{~g}^{-1}$ to $1195 \mathrm{~m}^{2} \mathrm{~g}^{-1}(-14 \%)$.
Consequently, when probed for $\mathrm{CO}_{2}$ and $\mathrm{N}_{2}$ adsorption, the volumetric uptake decreased by $14-18 \%$ for both gases: from 1.87 to $1.62 \mathrm{mmol} \mathrm{cm}{ }^{-3}$ for $\mathrm{CO}_{2}$ and from 0.27 to $0.23 \mathrm{mmol} \mathrm{cm}^{-3}$ for $\mathrm{N}_{2}$ at $25{ }^{\circ} \mathrm{C}$ and $1 \mathrm{MPa}$. Besides, they showed the capability of UiO-66 to separate $\mathrm{CO}_{2}$ over $\mathrm{N}_{2}$ with a factor of 6 .

Khabzina et $a l .{ }^{55}$ studied UiO-66-COOH and its performance for $\mathrm{NH}_{3}$ removal before and after shaping, using different techniques including extrusion, granulation and pelletization. When compressed at 9.7 MPa, the MOF exhibited a 14\% loss in BET surface area $-614 \mathrm{~m}^{2} \mathrm{~g}^{-1}$ as opposed to $710 \mathrm{~m}^{2} \mathrm{~g}^{-1}$ for the original powder. Importantly, such pellets outperformed the commercial $\mathrm{NH}_{3}$ adsorbents based on activated carbon (AC-3M and $\mathrm{AC}-\mathrm{Norit} \mathrm{RZN}_{1}$ ) in terms of both the total amount of adsorbed $\mathrm{NH}_{3}$ and the breakthrough time over a wide range of relative humidities (see Table 10 for detailed data).

\section{MIL-100 family}

Yang et al. ${ }^{56}$ addressed in their study the effect of the activation temperature on the adsorption properties of Cr-based MIL-100 in both powder and granular forms. The latter were prepared upon crushing and sieving compressed pellets (Fig. 2e and f). They found that activation at $300{ }^{\circ} \mathrm{C}$ under vacuum for $12 \mathrm{~h}$ led to partial collapse of the framework, as deduced from broader reflections with decreased intensities. Indeed, they proposed $250{ }^{\circ} \mathrm{C}$ as the optimal temperature for the MOF activation. Besides, it was demonstrated that at 1 bar and $25{ }^{\circ} \mathrm{C}$ the thusactivated MIL-100 powder adsorbed $5.8 \mathrm{mmol} \mathrm{g}^{-1} \mathrm{CO}_{2}$ - the highest reported value under the same conditions. This shows the crucial role of thermal activation in releasing the open $\mathrm{Cr}^{n+}$ sites which are responsible for the adsorption of $\mathrm{CO}_{2}$ molecules. In addition, the authors showed that once activated at $250{ }^{\circ} \mathrm{C}$ the MIL-100 pellets experienced a considerable loss in $\mathrm{CO}_{2}$ uptake, from 5.8 to $4.05 \mathrm{mmol} \mathrm{g}^{-1}$, suggesting poorer accessibility of open $\mathrm{Cr}^{n+}$ sites upon densification. Interestingly, by screening other gases the authors concluded that densification affected less the adsorption of $\mathrm{CH}_{4}$ and $\mathrm{N}_{2}$ over MIL-100 pellets as compared to $\mathrm{CO}_{2}$.

Valekar et al. ${ }^{57}$ compared the $\mathrm{NH}_{3}$ adsorption capacities of Fe-based MIL-100 pellets with granules made via wet granulation. The results suggested a partial loss of long order arrangement of the framework as well as a considerable loss in BET surface area, from $2088 \mathrm{~m}^{2} \mathrm{~g}^{-1}$ for the powder to $1300 \mathrm{~m}^{2} \mathrm{~g}^{-1}$ for the compressed pellet. The same detrimental effect of pelletization was observed for Cr-based MIL-101. Fe-MIL-100 pellets showed an $\mathrm{NH}_{3}$ uptake of $3.6 \mathrm{mmol} \mathrm{g}{ }^{-1}$, however, less than that of the MIL-100 granules $\left(4.4 \mathrm{mmol} \mathrm{g}^{-1}\right)$, also with a shorter breakthrough time.

\section{MOF-801}

Taddei et $a{ }^{58}{ }^{5}$ studied the influence of compression on the textural and adsorption properties of Zr-based MOF-801. They applied four different pressures (146, 292, 438 and $584 \mathrm{MPa})$ and found that the reflections in the XRD patterns became broader and less intense as the compression pressure increased, implying partial collapse of the framework. 

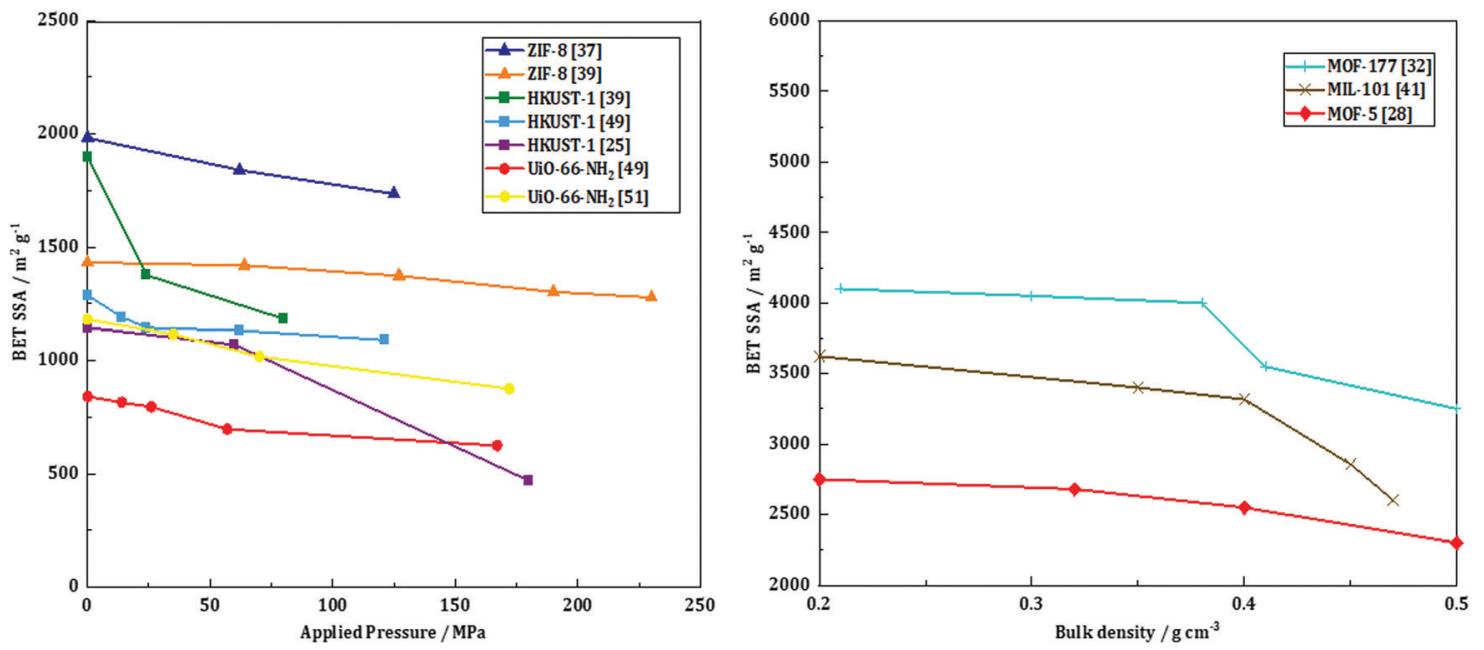

Fig. 3 BET SSA as a function of applied pressure during pelletization (left): ( $\mathbf{\Lambda})-\mathrm{ZIF}-8$ by Ribeiro et al.., ${ }^{37}(\mathbf{\Lambda})-\mathrm{ZIF}-8$ by Bazer-Buchi et al., ${ }^{39}$ ( 0 ) - UiO$66-\mathrm{NH}_{2}$ by Peterson et al., ${ }^{51}()-\mathrm{UiO}-66-\mathrm{NH}_{2}$ by Dhainaut et al., ${ }^{49}(\square)-$ HKUST-1 by Bazer-Buchi et al., ${ }^{39}(\square)-$ HKUST-1 by Dhainaut et al., ${ }^{49}$ and $(\square)-$ HKUST-1 by Alcañiz-Monge et al. ${ }^{25}$ BET surface area as a function of bulk density (right): (+) - MOF-177 by Zacharia et al., ${ }^{32}$ ( $X$ ) - MIL-101 by Ardelean et al., ${ }^{41}$ and $(\checkmark)-$ MOF-5 by Purewall et al. ${ }^{28}$

Interestingly, the compression time was shown to have no effect on the final crystallinity nor the mechanical resistance, assessed by repetitively dropping the pellets from a $36 \mathrm{~cm}$ height until they showed considerable damage. Therefore, the shortest time of $15 \mathrm{~s}$ was preferred. Besides, the addition of 5 wt $\%$ PVB as a binder had a positive effect on the mechanical properties, with higher resistance upon the drop test. The BET surface area of the pristine MOF powder, $899 \mathrm{~m}^{2} \mathrm{~g}^{-1}$, decreased significantly upon compression. There was, however, no significant difference between the pellets compressed at 146, 292 and $438 \mathrm{MPa}$ with $5 \mathrm{wt} \% \mathrm{PVB}$ as they exhibited BET surface areas of $569(-37 \%), 546(-39 \%)$ and $605 \mathrm{~m}^{2} \mathrm{~g}^{-1}(-33 \%)$, respectively. The pellets pressed with $5 \mathrm{wt} \% \mathrm{PVB}$ under 146 MPa for 15 seconds were further tested, and they retained $86 \%$ of the powder's $\mathrm{CO}_{2}$ gravimetric uptake as well as $74 \%$ of the powder's working capacity towards $\mathrm{H}_{2} \mathrm{O}$ adsorption.

To sum up, pelletization is a relatively easy and fast shaping technique which only requires a manual press. Depending on the targeted application, it can be performed with or without a binder. The former case enhances the mechanical stability of the final pellets, yet at the expense of a lower accessible surface area. The structural and textural integrity upon pelletization depends on the applied pressure and the nature of the MOF itself. Thus, several MOFs such as UiO-66- $\mathrm{NH}_{2}$ and ZIF-8 can undergo compression at high pressures, with relatively preserved intrinsic structural properties and minor losses in textural properties. For these MOFs, the loss in BET surface area as a function of applied pressure mostly presents a linear behavior (Fig. 3, left). This implies that for these MOF families pelletization can be considered as an appropriate way of shaping. At the same time, HKUST-1 has a more unpredictable trend, meaning that the textural properties of the final pellets depend as well on the shaping conditions (presence of moisture or linker in excess). Thus, special care should be given to the preparation of HKUST-1 pellets. Generally, pelletization leads to an increase of the bulk density, thus increasing the volumetric adsorption capacities - a crucial characteristic for gas adsorption applications. Once again, certain MOFs such as MOF-5, MOF-177 and MIL-101 demonstrate a linear trend of their bulk density as a function of BET surface area (Fig. 3, right). This highlights the growing potential of these particular MOFs in $\mathrm{H}_{2}$ storage.

\section{Granulation}

Granulation is a commonly-used shaping process which is based on the size enlargement of a parent powder material into an assembly of bigger agglomerates. A typical body shaped via granulation is referred to as a "granule" with an average size range of 2-20 mm..$^{59}$ Many productions such as the pharmaceutical and food industries rely on the utilization of such granules for processing and storage purposes. Notably, granulation is of great importance for the chemical industry as it allows shaping materials (adsorbents, catalysts) into granules which are subsequently used in fixed-bed reactors. This minimizes the risk of pressure drops during large-scale industrial productions, and ensure homogeneous diffusion. ${ }^{59}$

It should be noted that there are two types of granulation processes distinguished in the literature: wet and dry granulation. Dry granulation is applied when powders are incompatible with the use of solvents. Typically, it implies the compression of a parent powder at high pressures followed by mild crushing and sieving. Mainly, this process resembles and is typically subsequent to pelletization. Therefore, it was described in the previous part.

As the name suggests, wet granulation implies first wetting the initial powder. For this purpose, the powder is loaded in a high shear-rate mixer (Fig. 4a) and a volatile solvent 

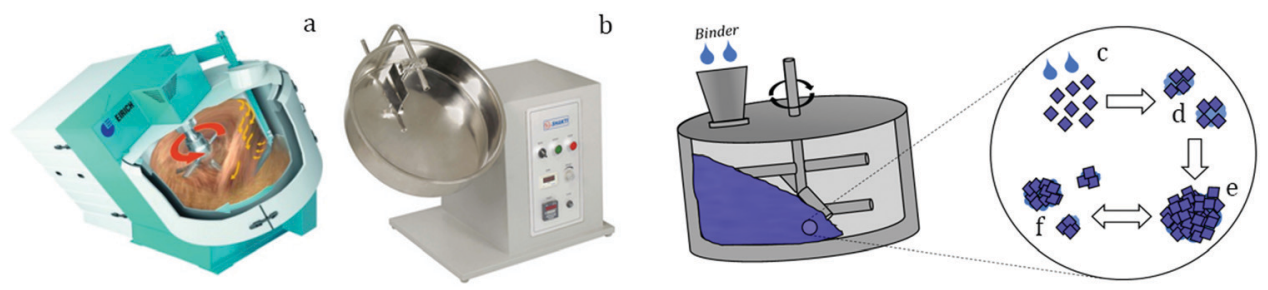

Fig. 4 Typical wet granulation equipment: a high shear-rate mixer (Maschinenfabrik Gustav Eirich GmbH \& Co KG), also referred to as a granulating pan (a) with an adjustable speed and direction of rotation; and a disc pelletizer (ERWEKA GmbH) also referred to as a rolling machine (b) with a controllable speed and inclination angle. Schematic representation of the wet granulation process: (c) mixing; (d) wetting and nucleation; (e) growth; and (f) spherization by attrition and breakage.

(i.e. short-chain hydrocarbons or water) is added in portions generally by spraying for homogeneity. This step is usually fast - a few tens of seconds to minutes - and causes wetting and cohesion of individual particles into primary agglomerates.

Once formed, the agglomerates are further transferred into a rolling machine (Fig. 4b), which finalizes the process by inducing continuous attrition between individual agglomerates over hours, thus facilitating and enhancing their spherical shape. Finally, the beads are dried, thus leading to pure granules of the parent material. The overall wet granulation process is presented in Fig. 4c-f.

However, upon solvent removal, the agglomeration of particles into spherical beads is only ensured by cohesion forces. Owing to their limited strength, the as-produced spheres may not have sufficient mechanical strength to maintain their shape and therefore be used for a targeted application. In this case, the reinforcement of the shaped granules by addition of binders is of particular interest. There are many types of binders known and used nowadays. Generally, they can be classified into inorganic binders such as clays,${ }^{60}$ silica, ${ }^{61}$ and alumina $^{62}$ and organic binders such as polyalcohols ${ }^{63}$ and carbohydrates. ${ }^{64}$ As for the former, not only do they improve the mechanical stability but they also contribute to the physical and chemical properties of the parent powder being shaped. Thus, upon shaping of zeolites with clays as binders there is an evolution of the physicochemical and thus catalytic properties of the initial zeolite. ${ }^{65}$ In the same fashion, alumina contributes to the acidic properties of zeolites. ${ }^{66}$ On the other hand, organic binders tend to improve the mechanical stability. Besides, they might be easily removed from the shaped composites upon thermal treatment, thus not altering the physical and chemical properties of the initial parent powder.

Table 6 List of MOFs shaped via wet granulation

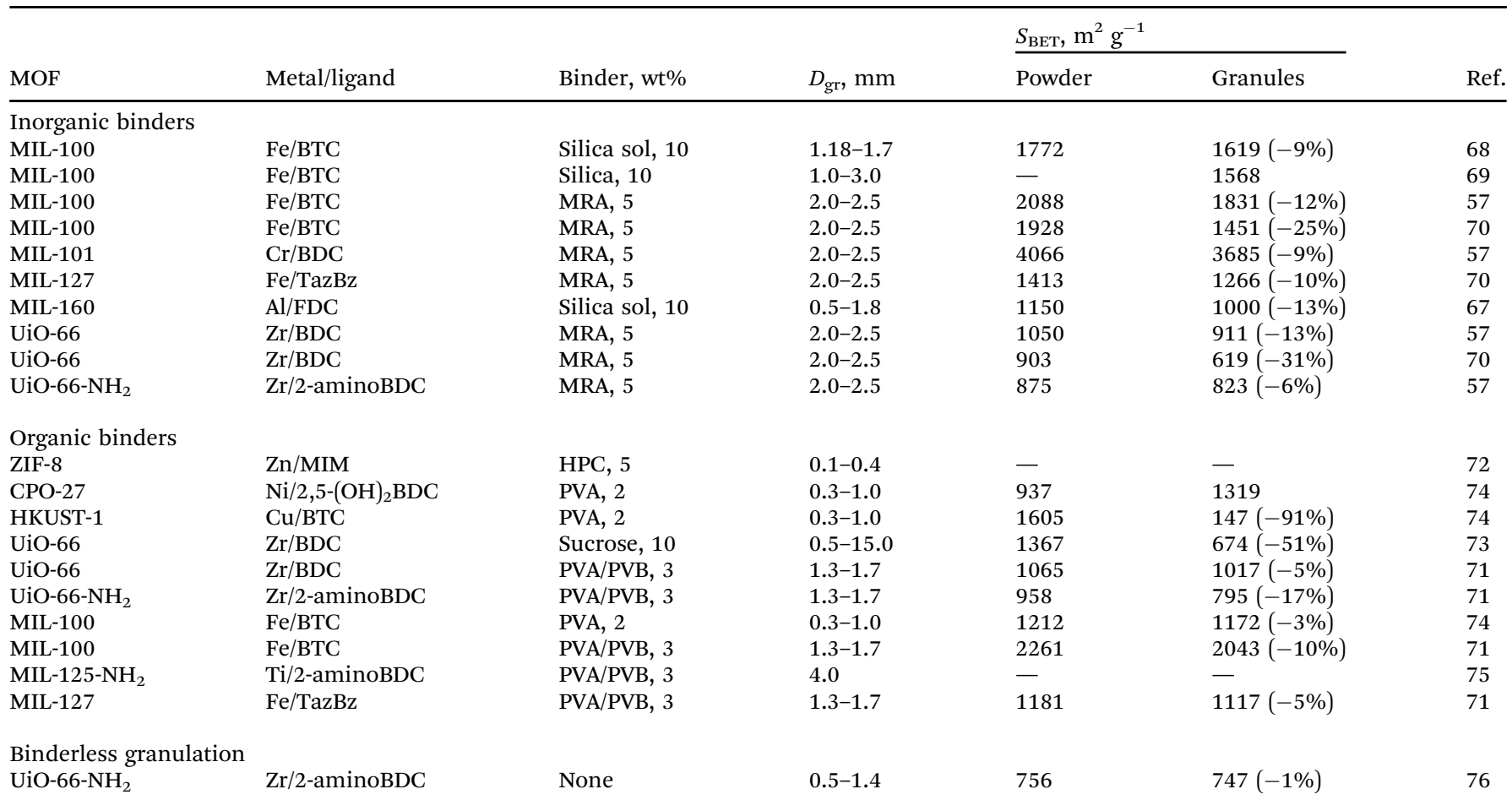

Ligand codes: BTC - benzene-1,3,5-tricarboxylic acid; BDC - benzene-1,4-dicarboxylic acid; FDC - 2,5-furandicarboxylic acid; TazBz - 3,3',5,5'azobenzenetetracarboxylate; and MIM - 2-methyl imidazole. Binder codes: PVA - polyvinyl alcohol; PVB - polyvinyl butyral; MRA - mesoporous $\rho$ alumina; and HPC - hydroxypropyl cellulose. "-" not specified. 
For MOFs, the primary goal of using binders is to enhance the mechanical stability of the granules. For this purpose, compounds capable of creating decently strong bonds with the surfaces of MOFs are preferred as binders. As an example, this implies cohesion of MOF particles via hydrogen bonding, and therefore, compounds possessing multiple functional groups (mainly - $\mathrm{OH}$ ) are beneficial. This includes alcohols (polyvinyl), sugars (sucrose, cellulose), esters (hydroxypropyl cellulose) and others. However, upon shaping they cannot be removed due to the limited thermal stability of MOFs.

It is worth mentioning that the overall properties of the shaped granules depend both on the features of the parent MOF powder and on the properties of the binder. Therefore, the choice of the latter is highly important as well as its total content in the final composition. Indeed, granulation of MOFs should yield compact and stable spheres with a minimum loss of their intrinsic properties including their specific surface area and pore volume. Therefore, the main objective of granulation is to find a balance between providing sufficient mechanical stability and preserving the initial textural properties of MOFs. The up-to-date list of thus-shaped MOFs is given in Table 6.

\section{Inorganic binders}

\section{Silica}

The effect of binders on the textural properties of shaped granules is evidenced in Table 6 . Indeed, there is a certain decrease of $S_{\mathrm{BET}}$ for all the studied MOFs; however, once shaped into granules, these MOFs are better fitted for a targeted application. Thus, in 2017 Permyakova et al. ${ }^{67}$ studied the heat-storage properties of a series of MOFs including MIL127(Fe), MIL-125(Ti)-NH $\mathrm{NH}_{2}$ MIL-100(Fe) and MIL-160(Al). The latter demonstrated one of the highest cycling loading lifts (the amount of water exchanged between adsorption and desorption steps) and therefore high energy storage capacity (the amount of energy stored per unit of mass of a material). Besides, the structural integrity of MIL-160 was preserved upon 10 adsorption/desorption cycles as compared to the other studied MOFs. Therefore, the authors further examined the performances of MIL-160 granules in a pilot-scale reactor. For a typical shaping via the wet granulation process, a predefined amount of MIL-160 powder was subjected to mixing with $10 \mathrm{wt} \%$ silica sol as a binder and subsequently granulation was finalized in a rolling machine to enhance the spherical shape. Eventually, the granules were dried at $100{ }^{\circ} \mathrm{C}$ for $12 \mathrm{~h}$ to remove the residual water and yield spherical objects of 0.5$1.8 \mathrm{~mm}$ (Fig. 5a).

The thus-shaped spheres were fully characterized, and according to their matching XRD patterns with the pristine powder, the crystal structure of the MIL-160 granules was assumed to be preserved. The morphology of the crystals was also preserved according to SEM images, with a secondary phase observed on the MOF surface attributed to the silica particles. Indeed, their presence was further proven by the
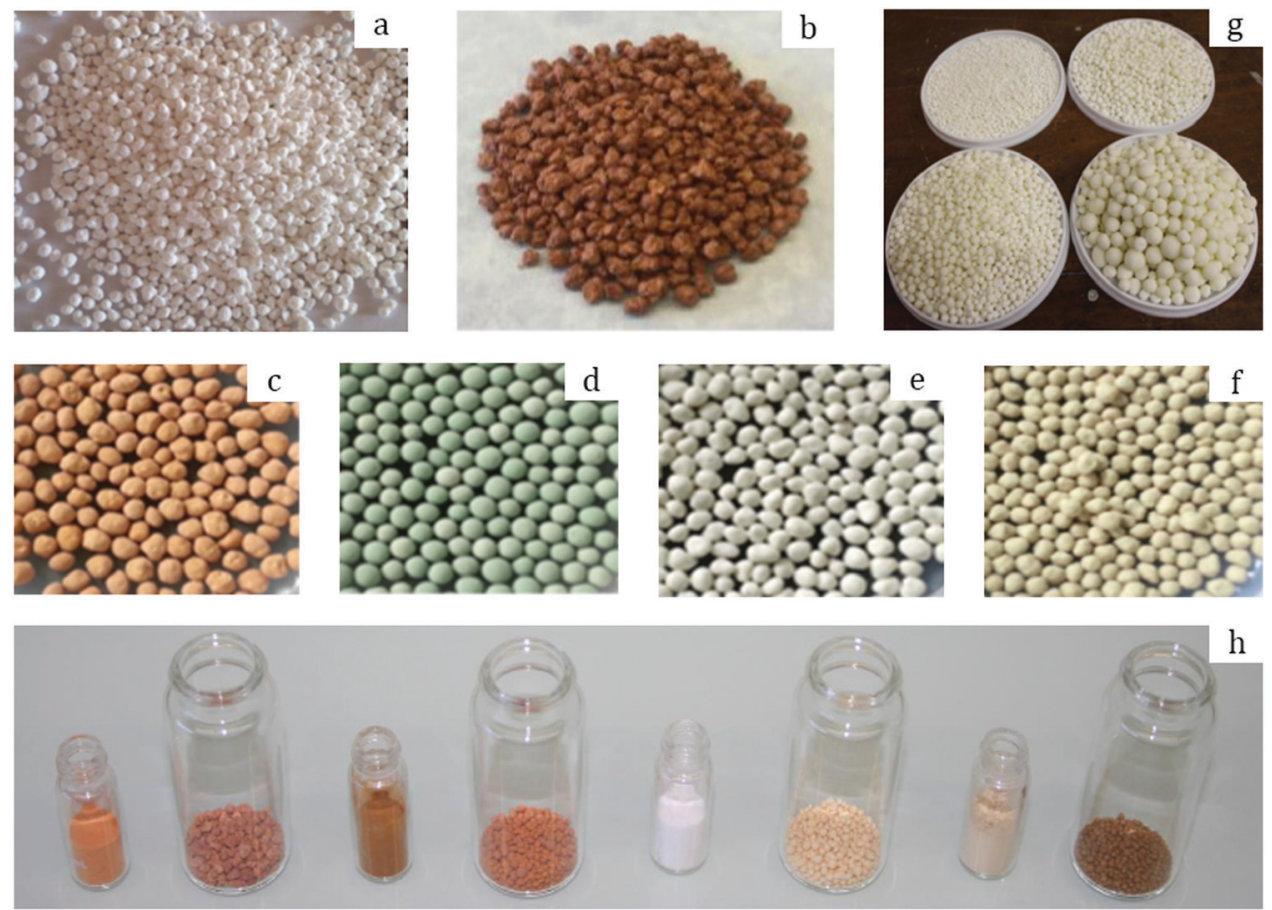

Fig. 5 Photographs of: MIL-160 granules (a) prepared by Permyakova et al. ${ }^{67}$ Copyright 2017 Wiley. MIL-100 granules (b) prepared by Martins et al. ${ }^{69}$ Copyright 2020 American Chemical Society. MIL-100 (c), MIL-101 (d), UiO-66 (e), and UiO-66- $\mathrm{NH}_{2}$ (f) containing 5 wt\% MRA binder prepared by Valekar et al. ${ }^{57}$ Copyright 2017 The Royal Society of Chemistry. UiO-66 granules (g) prepared by Ren et al. ${ }^{73}$ Copyright 2015 Elsevier. MIL-100, MIL-127, UiO-66, and $\mathrm{UiO}-66-\mathrm{NH}_{2}$ granules ( $\mathrm{h}$, from left to right in big vessels) with their corresponding powders in small vessels prepared by Chanut et al. ${ }^{71}$ Copyright 2016 Wiley. 
means of IR spectroscopy as an additional band observed in the spectrum of MIL-160 granules at $1120 \mathrm{~cm}^{-1}$, corresponding to $\mathrm{O}-\mathrm{Si}-\mathrm{O}$ asymmetric stretching. Importantly, $\mathrm{N}_{2}$ physisorption measurements revealed a loss in $S_{\mathrm{BET}}$ upon granulation, from 1150 to $1000 \mathrm{~m}^{2} \mathrm{~g}^{-1}$ for the parent MIL-160 powder and the corresponding granules, respectively. The same trend was observed for the total pore volume: $0.479 \mathrm{~cm}^{3} \mathrm{~g}^{-1}$ (powder) and $0.443 \mathrm{~cm}^{3} \mathrm{~g}^{-1}$ (granules). As the loss in specific surface area and pore volume $(-13 \%)$ slightly exceeded the binder content (10\%), partial pore blocking might occur. Nevertheless, the performance of the MOF MIL-160 towards cycling loading lifts was maintained, reaching 0.36 and $0.32 \mathrm{~g} \mathrm{~g}^{-1}(-11 \%)$, respectively, for the MIL-160 powder and granules measured between $30{ }^{\circ} \mathrm{C}$ (adsorption) and $80{ }^{\circ} \mathrm{C}$ (desorption) at $1.25 \mathrm{kPa}$. For the granules, this corresponded to an energy sorption capacity of $305 \mathrm{~W} \mathrm{~h} \mathrm{~kg}^{-1}$. This high value was obtained under relatively mild desorption conditions, making MIL-160 an excellent candidate for heat-storage applications.

The same binder was applied for the wet granulation of Febased MIL-100 by Kim et al. ${ }^{68}$ For a typical shaping procedure, they mixed $700 \mathrm{~g}$ of pre-synthesized MIL-100 with $10 \mathrm{wt} \%$ silica sol in a mixing granulator. Then, the primary agglomerates were transferred to a spheronizer to enhance the spherical shape. The thus-formed granules were dried at $110{ }^{\circ} \mathrm{C}$ for $12 \mathrm{~h}$ to remove any residual moisture. Eventually, this process yielded spherical granules with a size distribution of 1.18$1.70 \mathrm{~mm}$. These granules exhibited a similar XRD pattern to the original powder, suggesting that the shaping process had no impact on the crystal structure. In accordance with this, the spheres preserved most of the textural properties of the parent MOF powder, presenting specific surface areas of 1619 and $1772 \mathrm{~m}^{2} \mathrm{~g}^{-1}$ and total pore volumes of 0.82 and $0.93 \mathrm{~cm}^{3} \mathrm{~g}^{-1}$, respectively. The authors further applied the granules for the separation of $\mathrm{SF}_{6}$, which is a greenhouse gas, from a $\mathrm{N}_{2}$ stream. Noteworthily, the powder and granulated MIL-100 demonstrated nearly the same $\mathrm{SF}_{6}$ adsorption capacity of 1.673 and $1.658 \mathrm{mmol} \mathrm{g}^{-1}$, respectively. Thus, there was no considerable difference in the adsorption properties upon granulation. Interestingly, the adsorption capacity of the MIL-100 granules kept increasing with pressure, unlike the commercial adsorbent zeolite 13X. Indeed, the latter reached saturation at around 3 bar (with $2.5 \mathrm{mmol} \mathrm{g}^{-1}$ at $25{ }^{\circ} \mathrm{C}$ ), while the former demonstrated gradually increasing gas uptake, reaching around $4 \mathrm{mmol} \mathrm{g}^{-1}$ at 3 bar and $25{ }^{\circ} \mathrm{C}$. The authors attributed this phenomenon to the presence of mesopores in MIL-100, which contributed to the adsorption of $\mathrm{SF}_{6}$ once micropores were filled. Furthermore, although the $\mathrm{SF}_{6}$ breakthrough times were nearly identical for the MIL-100 granules and zeolite $13 \mathrm{X}$ granules at high pressure ( $9 \mathrm{bar}$ ), the desorption/regeneration of the former was considerably shorter $(20 \mathrm{vs} .250 \mathrm{~min})$ and complete, while the latter exhibited residual $\mathrm{SF}_{6}$ after regeneration due to strong interactions.

The same approach was also applied to shape MIL-100 by Martins et al. ${ }^{69}$ In a typical shaping procedure, the parent MIL100 powder was mixed with $10 \mathrm{wt} \%$ silica as a binder in a rolling machine. During mixing, water and ethanol were periodically sprayed on the blend to facilitate the agglomeration of individual particles. Eventually, the granules were isolated and dried at $100{ }^{\circ} \mathrm{C}$ to remove the residual solvents. This procedure resulted in semi-spherical granules with an average size of 1.0-3.0 mm (Fig. 5b), presenting a micropore volume of $0.58 \mathrm{~cm}^{3} \mathrm{~g}^{-1}$ and a specific surface area of $1568 \mathrm{~m}^{2} \mathrm{~g}^{-1}$, which is in agreement with Kim et al. ${ }^{68}$ The beads were further applied to ethane/propane and ethylene/propane gas mixture separation. The results suggested preferential $\mathrm{C}_{3} \mathrm{H}_{8}$ adsorption over $\mathrm{C}_{2} \mathrm{H}_{6}$ and $\mathrm{C}_{2} \mathrm{H}_{4}$. This remained the case when the temperature was varied, highlighting the potential of the MIL-100 granules for $\mathrm{C}_{2} / \mathrm{C}_{3}$ separation following pressure-swing adsorption (PSA). Moreover, lab-scale vacuum-swing adsorption (VSA) experiments starting from a 0.30 ethane $/ 0.70$ propane mixture, at $50{ }^{\circ} \mathrm{C}$ and $150 \mathrm{kPa}$, were conducted. The MIL-100 granules yielded an ethane-rich stream with a purity of $99.5 \%$ and a recovery of $86.7 \%$, as well as a propane-rich stream with a purity of $99.4 \%$ and a recovery of $97.0 \%$. The same VSA experiment starting from a 0.30 ethylene $/ 0.70$ propane mixture resulted in an ethylene-rich stream with a purity of $100 \%$ and a recovery of $75.8 \%$, as well as a propane-rich stream with a purity of $94.7 \%$ and a recovery of $100 \%$. The obtained results show that MOFs such as MIL-100 adequately shaped are highly promising for industrial separation processes.

\section{Mesoporous $\rho$-alumina (MRA)}

Another class of inorganic binders was first probed by Valekar et $a .^{57}$ for granulating a series of MOFs. They produced granules of MIL-100, MIL-101, UiO-66 and UiO-66- $\mathrm{NH}_{2}$ by mixing pre-defined amounts of MOF powders with 5-20 wt\% mesoporous $\rho$-alumina (MRA) in a rolling machine. During mixing, the blend was sprayed with water to facilitate particle agglomeration. The thus-produced granules were further sieved and rounded in a rolling machine. Finally, spheres with sizes of $2.0-2.5 \mathrm{~mm}$ were isolated and dried at $110{ }^{\circ} \mathrm{C}$ for $12 \mathrm{~h}$ (Fig. 5c-f).

The structural properties of the shaped granules were preserved for all the studied MOFs upon granulation. Indeed, the XRD pattern of each granular MOF matched well with its powder counterpart. Besides, the same observation applies to the textural properties as $\mathrm{N}_{2}$ physisorption measurements yielded similar isotherm shapes for MOF powders and granules, while the specific surface areas measured by the BET method suggested a consistent loss within the $1.0-7.7 \%$ range when shaped with $5 \mathrm{wt} \%$ MRA. Moreover, there was clear evidence of hierarchical meso- and macroporous structures confirmed by mercury porosimetry, due to the inter-particle voids generated upon granulation. Furthermore, the authors provided valuable information on the preservation of the MOFs' chemical properties after shaping. Indeed, $\mathrm{NH}_{3}$-TPD and pyridine-FTIR measurements performed on MIL-100 revealed the presence of Lewis acid sites in the temperature range of $150-200{ }^{\circ} \mathrm{C}$. They originated from unsaturated metal sites within the MOF structure, which were induced mainly upon thermal activation. The binder could partially block the accessibility to the acidic sites of the MOF; however, the 
$\mathrm{NH}_{3}$-TPD measurements suggested that these sites remained accessible upon granulation with no change in desorption peak positions. While the acidic strength remained equivalent, the loss in total acidic sites was consistent with the binder content $(5,10$ or $20 \mathrm{wt} \%)$. The same phenomenon was observed upon pyridine-FTIR measurements. The bands corresponding to pyridine coordination with $\mathrm{Fe}^{3+}\left(1014 \mathrm{~cm}^{-1}\right)$ and $\mathrm{Fe}^{2+}$ (1005 $\mathrm{cm}^{-1}$ ), responsible for the Lewis acidity in MIL-100, remained intact. Therefore, the authors demonstrated that granulation with the MRA binder had no effect on the acidic properties of MIL-100.

Interestingly, they also prepared MIL-100 pellets following the pelletization method and compared the thus formed bodies with the granules in terms of $\mathrm{NH}_{3}$ adsorption. The latter exhibited higher adsorption capacity at $25{ }^{\circ} \mathrm{C}(4.4 \mathrm{vs}$. $3.6 \mathrm{mmol} \mathrm{g}^{-1}$ ), suggesting that upon pelletization, the parent powder underwent more drastic structural and textural changes as compared to granulation. This was supported by XRD and $\mathrm{N}_{2}$ physisorption measurements.

A further study on the gas sorption properties of MOF granules was done by Iacomi et al. ${ }^{70}$ For this purpose, they followed the protocol proposed by Valekar et al. ${ }^{57}$ to form UiO66, MIL-100 and MIL-127 granules. The textural properties, as evaluated by $\mathrm{N}_{2}$ physisorption measurements, revealed a drastic decrease in specific surface area upon granulation of -10 , -25 , and $-31 \%$ for MIL-127, MIL-100 and UiO-66, respectively. At the same time, the total pore volume experienced a loss of $-26,-23$, and $-36 \%$, respectively. The obtained results were attributed to the possible structural degradation upon shaping. Indeed, the losses in textural properties were higher than the $5 \%$ reported in the previous study by Valekar et al. ${ }^{57}$ and might be due to the higher shear forces applied during the mixing step. Nevertheless, adsorption experiments performed both on the UiO-66 powder and on its granular counterpart (Fig. 5h) suggested higher volumetric uptakes for the latter towards small adsorbates such as $\mathrm{N}_{2}, \mathrm{CH}_{4}$ and $\mathrm{CO}_{2}$ as a result of densification upon granulation, as observed by Chanut et $a{ }^{71}{ }^{71}$ However, larger adsorbates such as $\mathrm{C}_{3} \mathrm{H}_{6}, \mathrm{C}_{3} \mathrm{H}_{8}$ and $\mathrm{C}_{4} \mathrm{H}_{10}$ experienced a decreased volumetric uptake. The authors attributed this phenomenon to the pore blocking effect of the binder. Interestingly, upon adsorption of $\mathrm{CO}$ and $\mathrm{C}_{3} \mathrm{H}_{6}$ on MIL127 and MIL-100, they reported higher adsorption enthalpies as compared to UiO-66. This indicates the presence of $\mathrm{Fe}^{2+}$ sites, generated upon thermal activation by partial reduction of $\mathrm{Fe}^{3+}$ sites. Indeed, $\mathrm{Fe}^{2+}$ sites induce stronger interactions with molecules with permanent dipole/quadrupole moments or $\pi$ electrons such as $\mathrm{CO}, \mathrm{CO}_{2}$ and $\mathrm{C}_{3} \mathrm{H}_{6}$.

Lastly, as a complementary study, Iacomi et al. ${ }^{70}$ performed water and methanol vapor adsorption experiments to address the effect of binders on the surface properties of UiO-66, MIL100 and MIL-127 MOFs. Generally, both $\mathrm{H}_{2} \mathrm{O}$ and $\mathrm{CH}_{3} \mathrm{OH}$ adsorption isotherms remained identical for all three MOFs upon shaping, suggesting that MRA showed no alteration of the hydrophilicity/hydrophobicity balance. Importantly, the slow initial water uptake on UiO-66 is consistent with a hydrophobic surface, while the opposite was observed for MIL-127, highlighting its hydrophilic surface; at the same time, MIL100 presented intermediate surface hydrophilicity/hydrophobicity features.

\section{Organic binders}

\section{Carbohydrate-based binders}

Ohsaki et al. $^{72}$ showed the possibility to shape ZIF-8 powder in a mixing granulator using two different approaches: liquid or powder addition. The former implied mixing the ZIF-8 powder with a few consecutive portions of an aqueous solution of the binder hydroxypropyl cellulose and successive steps of mixing for $5 \mathrm{~min}$. The powder addition approach, on the other hand, suggested initial mixing of ZIF-8 with the binder in its dry form ( $5 \mathrm{wt} \%$ ) followed by the subsequent addition of portions of water (42 wt\%) during mixing. Interestingly, the latter was found to be more suitable as it yields granules with an average size of $>200 \mu \mathrm{m}$, while the liquid addition method resulted in a large percentage of non-granulated ZIF- 8 particles. The authors attributed this phenomenon to a more uniform mixing of the MOF with the binder upon the powder addition method which was consequently chosen for the rest of the study, and this highlights the importance of mixing the powder and the binder prior to granulation. The XRD results suggested the preservation of the crystal structure upon granulation as evidenced from the matching patterns of the ZIF-8 powder and the granules. Furthermore, the granules retained a consequent specific surface area, around $90 \%$ of the original powder. Noteworthily, the effect of the binder content on the surface area was addressed in the study. Namely, upon increasing the binder percentage up to $20 \mathrm{wt} \%$ (at a constant amount of water of $42 \mathrm{wt} \%$ ) the granules retained around $60 \%$ of the initial surface area. The decrease became more drastic at $30 \mathrm{wt} \%$ binder loading as the granules retained $<10 \%$ of the starting surface area. This set the upper limit for the binder content at around $20 \mathrm{wt} \%$. At the same time, the water content was shown to have a low impact on the surface area.

Another example of wet granulation with carbohydrates as binders was provided by Ren et al. ${ }^{73}$ who described the shaping of UiO-66 with sucrose. More precisely, they mixed $450 \mathrm{~g}$ of MOF with $50 \mathrm{~g}$ of sucrose in a centrifugal granulator followed by the spraying of water. The granules were further subjected to drying at $60{ }^{\circ} \mathrm{C}$ for $24 \mathrm{~h}$. The entire procedure yielded spherical beads with an average size of 0.5-15 mm (Fig. $5 \mathrm{~g}$ ).

As confirmed by XRD, the crystal structure remained intact upon granulation. The presence of the binder was assumed as a secondary plate-like phase was observed in the SEM images. Consequently, there was an evident impact on the textural properties of the UiO-66 granules brought about by the binder. Namely, the specific surface area decreased to $674 \mathrm{~m}^{2} \mathrm{~g}^{-1}$, which represents $50 \%$ of the SSA of the parent powder. Accordingly, the total pore volume decreased from 0.56 to $0.34 \mathrm{~cm}^{3} \mathrm{~g}^{-1}$. In agreement with that, the hydrogen uptake similarly experienced a coherent decrease, from $1.54 \mathrm{~cm}^{3} \mathrm{~g}^{-1}$ for the UiO-66 powder to $0.85 \mathrm{~cm}^{3} \mathrm{~g}^{-1}$ obtained for the 
granules. Importantly, the authors provided data on the mechanical stability of the granulated UiO-66 based on nonconventional drop tests. Thus, no breakage was observed when dropping the granules on a steel surface from $0.5 \mathrm{~m}$ height after 70 consecutive drops. Moreover, attrition tests revealed that only $5 \%$ of the initial granule weight ended up as "fines", after $60 \mathrm{~min}$ of tumbling at $25 \mathrm{rpm}$ and further sieving. This suggested a considerable mechanical stability of the shaped granules.

\section{PVA and PVB binders}

Another class of binders largely used for wet granulation is polyalcohols, such as polyvinyl alcohol (PVA), and their derivatives, including polyvinyl butyral (PVB). The former was used in a study by Hindocha et $a .^{74}$ who formulated three MOFs (Cu-BTC (HKUST-1), CPO-27 and MIL-100) into spherical granules. The typical procedure implied pre-mixing $1 \mathrm{~g}$ of $\mathrm{MOF}$ powder with $2 \mathrm{wt} \%$ PVA followed by granulation upon addition of $0.25 \mathrm{~mL}$ of water. This formulation yielded spheres of 0.3-1.0 $\mathrm{mm}$ on average after sieving. As suggested by XRD results, this procedure had a considerable impact on the HKUST-1 framework, as the granules presented a pattern combining several mixed phases which were absent for the parent powder. In agreement with that, the shaped material showed a considerable decrease in specific surface area upon granulation, from 1605 to $147 \mathrm{~m}^{2} \mathrm{~g}^{-1}$ for the parent powder and the granules, respectively. Consequently, this material, losing its MOF structure, was not able to retain a similar ammonia adsorption capacity, reaching only $19 \mathrm{mg} \mathrm{g}^{-1}$, while the parent powder could adsorb up to $105 \mathrm{mg} \mathrm{g}^{-1}$ under the same conditions (500 ppm ammonia, 40\% RH). Thus, using water to shape HKUST-1 following wet granulation cannot be considered as an appropriate method.

On the other hand, the CPO-27 and MIL-100 frameworks proved to be more stable under the applied conditions, as the granules' diffractograms yielded matching patterns with their powder counterparts. The MIL-100 granules presented only a slight decrease in SSA $\left(S_{\mathrm{BET}}=1172 \mathrm{~m}^{2} \mathrm{~g}^{-1}\right)$, which is in the range of $2 \%$ loss as compared to the parent powder $\left(S_{\mathrm{BET}}=\right.$ $\left.1212 \mathrm{~m}^{2} \mathrm{~g}^{-1}\right)$, consistent with the initial amount of the binder. Surprisingly, the CPO-27 granules exhibited a considerable increase in specific surface area $\left(S_{\mathrm{BET}}=1319 \mathrm{~m}^{2} \mathrm{~g}^{-1}\right)$ as compared to $S_{\mathrm{BET}}=937 \mathrm{~m}^{2} \mathrm{~g}^{-1}$ of the as-synthesized CPO-27. This phenomenon was stated to be unclear by the authors.

Both granules exhibited an ammonia adsorption capacity of about $50 \mathrm{mg} \mathrm{g}^{-1}$ and a corresponding breakthrough time of about $100 \min \left(\left[\mathrm{NH}_{3}\right]=12.5 \mathrm{ppm}\right.$, Table 7$)$. For comparison, a

Table 7 Adsorptive and mechanical properties of the MOF granules prepared by Hindocha et al. ${ }^{74}$

\begin{tabular}{llll}
\hline MOF & $\begin{array}{l}\mathrm{NH}_{3} \text { adsorption } \\
\text { capacity, } \mathrm{mg} \mathrm{g}^{-1}\end{array}$ & $\begin{array}{l}\text { Breakthrough time, } \\
\text { min under 12.5 ppm } \\
\text { of } \mathrm{NH}_{3}\end{array}$ & $\begin{array}{l}\text { Weight loss } \\
\text { upon attrition } \\
\text { test, \% }\end{array}$ \\
\hline CPO-27 & 51 & 106 & 18 \\
MIL-100 & 50 & 100 & 2 \\
HKUST-1 & 19 & 43 & 11
\end{tabular}

commercial activated carbon presents an ammonia adsorption capacity of $8 \mathrm{mg} \mathrm{g}^{-1}$ with a breakthrough time of $9 \mathrm{~min}$. Interestingly, the authors performed attrition tests to probe the mechanical stability of the granules. Upon rolling in a mill for $18 \mathrm{~h}$ and subsequent sieving, the MIL-100 granules lost only $2 \%$ of their initial weight, while the CPO-27 granules were more affected $(-18 \%)$. Notably, the granules presented a reduced size due to the long-term attrition $(0.3-1.0 \mathrm{~mm})$. For comparison, the authors also performed dry granulation (binderless pelletization then milling-sieving) which was beneficial in the case of CPO-27 as it resulted in a higher ammonia adsorption capacity (64 vs. $51 \mathrm{mg} \mathrm{g}^{-1}$ ) as well as an extended breakthrough time (189 vs. $106 \mathrm{~min}$ ). On the other hand, the "dry-shaped" MIL-100 granules presented similar performances to the "wet-shaped" granules. Therefore, the choice of either shaping process depends on the MOF itself and its targeted application.

A mixture of PVA and PVB was used as a binder in the study by Chanut et al. ${ }^{71}$ The authors first mixed $5 \mathrm{~g}$ of MOF powder with a $3 \mathrm{wt} \%$ polymer blend, followed by periodical spraying of ethanol for a total of $50 \mathrm{~mL}$ to cause primary particle agglomeration. Upon sieving, a fraction with sizes between 1.3 and $1.7 \mathrm{~mm}$ (Fig. 5h) was rounded using a rolling device to achieve the final shape. Eventually, the spheres were dried at $110{ }^{\circ} \mathrm{C}$ for $12 \mathrm{~h}$ to remove the residual ethanol.

By analyzing the XRD patterns, a slight degradation of UiO$66-\mathrm{NH}_{2}$ and MIL-100 structures can be deduced from the decrease in the intensities of the principal reflections as compared to their powder counterparts. This was further supported by $\mathrm{N}_{2}$ physisorption measurements which revealed a certain decrease of their textural properties due to a partial structural collapse. At the same time, the corresponding losses in specific surface area and micropore volume for the UiO-66 and MIL-127 granules are within 3\%, which is in agreement with the binder content. In terms of adsorption properties, there is a general trend observed: after granulation, the gravimetric uptake decreased (less gas adsorbed per weight of granules than that per weight of powder), while the volumetric uptake increased (more gas adsorbed per volume of granules than that per volume of powder). This implies that the wet granulation process caused densification and therefore an increase in bulk density. The latter relates to the amount of adsorbent stackable in a reactor vessel and thus is an important factor when evaluating adsorption capacities for real-life applications.

Probing molecules with and without dipole/quadrupole moments such as $\mathrm{CO}, \mathrm{CO}_{2}$, and $\mathrm{CH}_{4}$ on both powder and granular UiO-66 and UiO-66- $\mathrm{NH}_{2}$ yielded similar adsorption profiles. This implies that granulation had no significant impact on the surface properties of the MOFs. Interestingly, the adsorption of larger butane molecules on MIL-100 granules revealed an " $S$ " shape isotherm with a sharp increase at elevated pressures, while a Langmuir-type adsorption curve was observed with the powder. The authors attributed this phenomenon to high partial pressures required to displace the polymeric chains of PVA/PVB and open the pores for butane adsorption. Notably, using the MIL-127 powder, the enthalpy 
profile of CO adsorption showed a total decrease along with a considerable drop at initial adsorption energies, while a linear behavior was obtained with MIL-127 granules. The authors attributed this phenomenon to the inaccessibility of Lewis acid sites after granulation of MIL-127. It possibly originated from the binder presence partially preventing $\mathrm{Fe}^{3+}$ reduction to $\mathrm{Fe}^{2+}$ during the thermal activation of MIL-127. However, as mentioned by the authors, more experiments are needed to further address this phenomenon.

A similar wet granulation methodology was applied by Regufe et al. ${ }^{75}$ Namely, the same binder system (3 wt\% PVA/ PVB blend) was mixed with Ti-based MIL-125_NH $\mathrm{NH}_{2}$ and further agglomerated in a pan-type granulation with the addition of an ethanolic solution. Ti-based MOF granules with an average size of $4.0 \mathrm{~mm}$ were further applied for gas adsorption and syngas purification. Adsorption measurements of numerous gases resulted in the following sequence of adsorption capacities: $\mathrm{CO}_{2}>\mathrm{CH}_{4}>\mathrm{N}_{2}>\mathrm{CO}>\mathrm{H}_{2}$ with 8.5, 7.1, 5.4, 3.5 and $0.3 \mathrm{~mol} \mathrm{~kg}^{-1}$, respectively. The authors further conducted pressure-swing adsorption (PSA) experiments on these granules. Interestingly, the hydrogen purification from a $\mathrm{CO} / \mathrm{H}_{2}$ $(30 \% / 70 \%)$ mixture reached $100 \%$. In a different experiment, starting from a mixture containing $30 \% \mathrm{CO}_{2}, 22 \% \mathrm{CO}$ and $48 \%$ $\mathrm{H}_{2}$, a suitable $\mathrm{CO} / \mathrm{H}_{2}$ mixture for the Fischer-Tropsch process, with $\mathrm{CO}_{2}$ impurity down to $3 \%$ was obtained.

\section{Binderless granulation}

So far, only one successful example of binderless MOF granules has been reported. Leloire et al. prepared UiO-66- $\mathrm{NH}_{2}$ granules by the stepwise addition of a water/ethanol mixture in a high shear-rate mixer, followed by the use of a rolling machine to both reinforce their mechanical resistance and increase their sphericity. ${ }^{76}$ After drying, well-rounded granules of 0.5 to $1.4 \mathrm{~mm}$ were obtained. Interestingly, their BET surface area was close to that of the powder (747 vs. $756 \mathrm{~m}^{2} \mathrm{~g}^{-1}$ ). Moreover, the authors observed by FT-IR spectroscopy the disappearance of a band at $1686 \mathrm{~cm}^{-1}$, attributed to the complexation of unsaturated zirconium cations at the surfaces of the UiO-66$\mathrm{NH}_{2}$ crystallites with free carboxylic groups. Of note, the vibration band at $1660 \mathrm{~cm}^{-1}$, typically attributed to the presence of residual DMF entrapped, decreased after granulation. Hence, partial extraction of DMF molecules during the shaping step occurred. As expected, the granules' average compressive strength remained low $(0.3 \mathrm{~N})$. They were nonetheless subjected to gamma irradiation and steam, after which they demonstrated slightly higher compressive strengths ( 0.5 and $0.4 \mathrm{~N}$, respectively) and BET surface areas (770 and $1051 \mathrm{~m}^{2} \mathrm{~g}^{-1}$, respectively). Finally, the granules were charged with $7.8 \mathrm{mg}$ of labelled radioiodine per gram of MOF, then subjected to conditions mimicking a severe nuclear accident (30 hours at $120{ }^{\circ} \mathrm{C}$ under a pressure of $3.5 \mathrm{bar}$ of humid air (R.H. $=20 \%$ ) and ${ }^{60}$ Co irradiation). No loss of ${ }^{131} \mathrm{I}_{2}$ was observed.

As a conclusion, granulation is a widely used shaping technique to produce spherical beads of a few millimeters in diameter. Granulation is only possible through the addition of solvents and binders, which facilitate coagulation between the individual MOF particles. Therefore, special care should be given to the MOFs whose structure might collapse upon addition of a solvent (water or alcohols). For example, HKUST-1 undergoes hydrolysis in the presence of moisture; hence its granulation can only be done under restricted conditions. In turn, binders are divided into inorganic and organic and serve to reinforce the mechanical stability of the final beads. Depending on their nature and amount, they can strongly affect the textural and chemical properties of the original MOF powders. Some of the features are highlighted in Table 8.

\section{Extrusion}

Extrusion is another important manufacturing process used in many industrial productions such as metallurgy, plastics, food, and pharmaceuticals. Besides, extrusion is considered to be the most commonly used technique for shaping catalysts and adsorbents for industrial applications in fixed-bed reactors. As the main principle, extrusion relies on passing the preformed paste inside an extruder through a die. Depending on the form of the die, the final objects can have different shapes: hollow tubes, sheets, strips, or cylinders with various crosssections. This wide variety of final morphologies makes extrusion an advantageous shaping technique as compared to pelletization or granulation. A typical body shaped via extrusion is referred to as an extrudate. Generally, in order to formulate a parent powder into extrudates, it is necessary to first suspend it in a liquid (usually water) to form a paste. The thus-formed paste is further transferred into an extruder where it is pushed with a piston/ram or a screw towards the die (Fig. 6). Upon passing the die, the extrudates (sheets, cylinders) are cut into pieces of desired length and subsequently dried to remove the residual solvent.

Table 8 Types of binders and their effect on the properties of pristine MOF powders

\begin{tabular}{|c|c|c|c|c|c|}
\hline MOF & Binder, wt $\%$ & \multicolumn{3}{|c|}{ Effect of the binder on different properties } & Ref. \\
\hline Fe-MIL-100 & $\rho$-Alumina, 5 & Crushing strength of $6.7 \mathrm{~N}$ & $\begin{array}{l}\sim 12 \% \text { loss of BET SSA, } \\
\text { higher than binder content }\end{array}$ & $\begin{array}{l}\text { No alteration of acidic properties, } \\
\text { preservation of Lewis acid sites }\end{array}$ & 57 \\
\hline UiO-66 & Sucrose, 10 & $\begin{array}{l}5 \% \text { breakage upon tumbling } \\
\text { after } 60 \mathrm{~h} \text { at } 25 \mathrm{rpm}\end{array}$ & $50 \%$ loss of BET SSA & $45 \%$ loss of $\mathrm{H}_{2}$ adsorption capacity & 73 \\
\hline Fe-MIL-100 & PVA, 2 & $\begin{array}{l}\text { Only } 2 \% \text { weight loss upon } \\
\text { attrition for } 18 \mathrm{~h} \text { in a mill }\end{array}$ & $\begin{array}{l}3 \% \text { loss of BET SSA, } \\
\text { consistent with binder content }\end{array}$ & $\begin{array}{l}\text { Higher } \mathrm{NH}_{3} \text { adsorption capacity } \\
\text { than that after pelletization }\end{array}$ & 74 \\
\hline
\end{tabular}



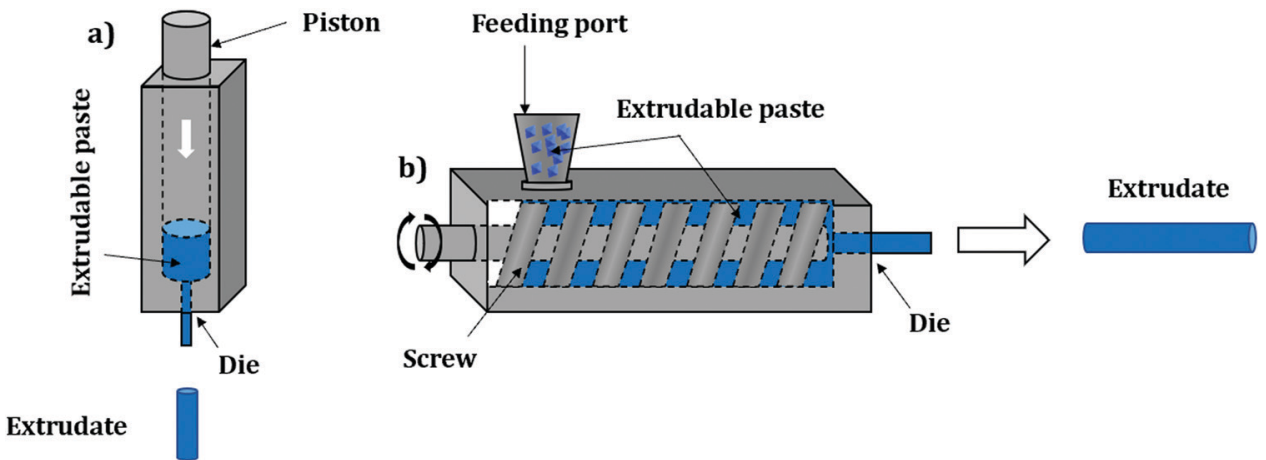

Screw

Fig. 6 Schematic representation of the extrusion process on a piston-type extruder (a) and on a single screw-type extruder (b).

Generally, extruders are divided into screw and piston types. The former allows continuous processing and might consist of one (single screw), two (twin screw) or multiple screws which operate in simultaneous and parallel rotations. On the other hand, piston extruders operate in batch mode; however, they enable the extrusion of pastes with high viscosity and compaction.

The paste formulation is crucial and requires special attention. Indeed, mixing of the parent powder with a liquid should yield a paste with suitable rheological properties to enable extrusion. There are many aspects which define the flow behavior such as the size and shape of the powder particles, their chemical properties, etc. Overall, the paste viscosity is dictated by the liquid content and can be decreased upon increasing the total liquid/solid ratio. More viscous pastes might require higher pressures for displacement within an extruder; however, unlike pelletization, extrusion does not affect as much the compaction of the particles as they are suspended in a liquid. Besides, in some cases the flowability, plasticity, or ability of the paste to withstand deformation upon extrusion can be enhanced by adding plasticizers. These are typical organic compounds based on cellulose or polyalcohols which facilitate the formation of the overall network. Generally, they are removed from the final extrudate composition by calcination.

As with granulation, extrusion implies the addition of binders to ensure sufficient mechanical strength to extrudates by developing cross-linking forces between the individual particles. Therefore, the choice of binder and its content are governed by the same principles as in the case of granulation and pelletization. Namely, the binder should provide sufficient mechanical stability with the minimum loss of intrinsic physical and chemical properties of the parent powder. Thus, zeolites extruded with clays and boehmite as binders and cellulose-based plasticizers experienced certain alterations of textural and chemical properties as well as a clear enhancement of mechanical resistance. ${ }^{77}$

Removal of organic components (plasticizer and binder) from extrudates strongly depends on the thermal stability of

Table 9 List of MOFs shaped via extrusion

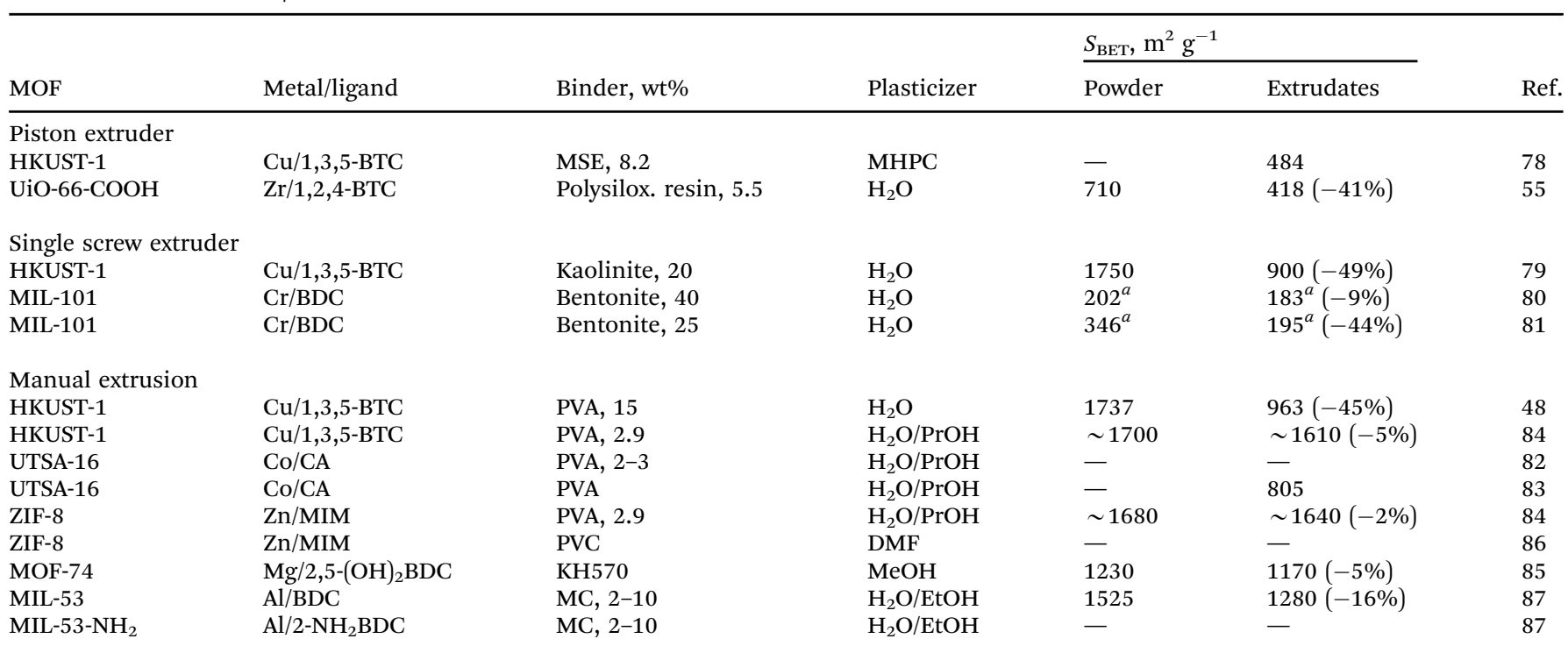

Ligand codes: 1,3,5-BTC - benzene-1,3,5-tricarboxylic acid; 1,2,4-BTC - benzene-1,2,4-tricarboxylic acid; BDC - benzene-1,4-dicarboxylic acid; CA citric acid; and MIM - 2-methyl imidazole. Binder codes: MSE - methoxy-siloxane ether; PVA - polyvinyl alcohol; PVC - polyvinyl chloride; KH570 3 (trimethoxysilyl)propyl methacrylate; and MC - methyl cellulose. Plasticizer codes: MHPC - methyl hydroxyl propyl cellulose and DMF - $N, N$ dimethylformamide. "-_" not specified. ${ }^{a}$ Measured by Hg intrusion. 
the parent powder. Zeolites, alumina, and silica are able to withstand calcination and therefore organic removal without structural changes. However, this is not the case for MOFs. Hence, upon extrusion, binders and plasticizers are usually retained in the extrudates due to the limited thermal stability of MOFs (Table 9).

\section{Piston extruder}

The pioneering work on MOF extrusion was made by Küsgens et $a l .{ }^{78}$ They successfully shaped HKUST-1 using a ram extruder by mixing the pre-synthesized MOF powder with methoxysiloxane ether $\left(\mathrm{CH}_{3} \mathrm{Si}(\mathrm{O})_{1.1}\left(\mathrm{OCH}_{3}\right)_{0.8}\right)$ as a binder and methyl hydroxyl propyl cellulose as a plasticizer. They proposed an optimal composition of the paste for the forming process: $180 \mathrm{~g}$ of MOF, $80 \mathrm{~g}$ of water, $25 \mathrm{~g}$ of binder, and $20 \mathrm{~g}$ of plasticizer. The paste was subsequently extruded and cut into $200 \mathrm{~mm}$-long strings which were dried at $120{ }^{\circ} \mathrm{C}$ for several hours to remove the residual water. The XRD pattern of the thus-shaped extrudates matches with that of the parent HKUST-1 powder, indicating the preservation of the MOF crystal structure upon extrusion in the presence of water. However, $\mathrm{N}_{2}$ physisorption resulted in a $S_{\mathrm{BET}}$ of $484 \mathrm{~m}^{2} \mathrm{~g}^{-1}$, which is fairly low for this MOF even when taking into account $36 \mathrm{wt} \%$ of organic additives and suggests either partial hydrolysis of the HKUST-1 framework or pore blockage.

Khabzina et al. also studied the use of UiO-66-COOH extrudates for $\mathrm{NH}_{3}$ removal. ${ }^{55}$ Extrusion was accomplished in a piston extruder through a $1.5 \mathrm{~mm}$ die. For a typical formulation, the predefined amount of the parent MOF (72.5 vol\%) was mixed with a polysiloxane-based resin as a binder (5.5 vol\%) and water (22 vol\%). The thus-formed paste was further transferred into the extruder to produce $300 \mathrm{~mm}$ long extrudates. They were further dried at room temperature and cut into $\sim 1.5 \mathrm{~mm}$ length. According to $\mathrm{N}_{2}$ physisorption measurements, the extrudates retained around $60 \%$ of the initial specific surface area upon shaping the parent powder, making up 418 and $710 \mathrm{~m}^{2} \mathrm{~g}^{-1}$, respectively. The core of the study was the exceptional performance of the extrudates for $\mathrm{NH}_{3}$ capture as compared to commercial activated carbon-based (AC) adsorbents. The UiO-66- $\mathrm{COOH}$ extrudates reached, respectively, 40 and $34 \mathrm{mg} \mathrm{cm}^{-3}$ of volumetric $\mathrm{NH}_{3}$ uptake at 0 and $40 \%$ relative humidity when subjected to a flow of $\mathrm{N}_{2}$ containing an $\mathrm{NH}_{3}$ concentration of $1200 \mathrm{ppm}$. At the same time, the commercial AC exhibited 20 and $29 \mathrm{mg} \mathrm{cm}^{-3}$, respectively. The same trend was observed for the breakthrough time, reaching 48 and 43 minutes for the MOF extrudates, against 21 and 32 minutes for the AC. Besides, the authors stated a considerable resistance of the extrudates to attrition, with a mass loss of $1.4 \%$ upon sieving (Table 10).

Interestingly, the authors also performed other types of shaping on UiO-66-COOH. As seen from Table 10, not only the extrudates but also the pellets compressed at 97 bar outperformed the commercial adsorbent (AC). At the same time, the relatively poor performance of the beads made via freeze granulation was attributed to their low bulk density.
Table 10 Adsorptive and mechanical properties of $\mathrm{UiO}-66-\mathrm{COOH}$ shaped bodies ${ }^{55}$

\begin{tabular}{|c|c|c|c|c|c|}
\hline \multirow[b]{2}{*}{ Adsorbent } & \multicolumn{2}{|c|}{$\begin{array}{l}\mathrm{NH}_{3} \text { uptake at } \\
1200 \mathrm{ppm}^{-3} \\
\mathrm{mg} \mathrm{cm}\end{array}$} & \multicolumn{2}{|c|}{$\begin{array}{l}\text { Breakthrough } \\
\text { time, min }\end{array}$} & \multirow[b]{2}{*}{ Attrition, ${ }^{a} \%$} \\
\hline & $0 \% \mathrm{RH}$ & $40 \% \mathrm{RH}$ & $0 \% \mathrm{RH}$ & $40 \% \mathrm{RH}$ & \\
\hline Extrudates & 40 & 34 & 48 & 43 & 1.4 \\
\hline Compressed pellets & 33 & 33 & 41 & 40 & 1.7 \\
\hline Beads & 14 & 16 & 18 & 20 & 0 \\
\hline Commercial AC & 20 & 29 & 21 & 32 & 0.2 \\
\hline
\end{tabular}

${ }^{a}$ Attrition tests were performed by rotating a cylinder containing a baffle and the shaped UiO-66-COOH at $60 \mathrm{rpm}$ for $30 \mathrm{~min}$. The percentage corresponds to the total mass of the fine particles - less than $425 \mu \mathrm{m}$ - after sieving.

\section{Single screw extruder}

Studies on HKUST-1 extrusion were also performed by Majano and Pérez-Ramírez. ${ }^{79}$ A HKUST-1 powder with a specific surface area of $1750 \mathrm{~m}^{2} \mathrm{~g}^{-1}$ was mixed with $20 \mathrm{wt} \%$ kaolin as a binder and water was used to induce paste formation. The paste was further pushed through a $2 \mathrm{~mm}$ die using a single screw extruder, and the extrudates were subsequently dried at $65{ }^{\circ} \mathrm{C}$ overnight. As confirmed by XRD, the crystal structure was retained upon extrusion, but $\mathrm{N}_{2}$ physisorption revealed a $S_{\mathrm{BET}}$ of $900 \mathrm{~m}^{2} \mathrm{~g}^{-1}$. This value is around $50 \%$ of the initial surface area of the HKUST-1 powder and indicates considerable pore blocking caused by the binder, and/or partial framework hydrolysis.

A study by Hong et al. ${ }^{80}$ was conducted on the extrusion of Cr-based MIL-101 for $\mathrm{CO}_{2}$ adsorption, using a single screw extruder and bentonite clay as a binder. A predefined amount of MIL-101 powder was mixed with bentonite clay (40 wt\%) and water to form a paste. The paste was further extruded into monoliths with square channels which were subsequently dried at $10{ }^{\circ} \mathrm{C}$ for several days followed by firing at $150{ }^{\circ} \mathrm{C}$ for $33 \mathrm{~h}$. Upon firing, the extrudates were cut into $7 \mathrm{~cm}$ long cylinders (Fig. 7a). According to the XRD patterns, the MIL-101 crystal structure was retained upon processing. However, the extrudates demonstrated reflections with considerably lower intensities, which was attributed by the authors to the binder's presence. Moreover, the extrudates experienced a small decrease of their textural properties, yielding a specific surface area of $183 \mathrm{~m}^{2} \mathrm{~g}^{-1}$, while it was $202 \mathrm{~m}^{2} \mathrm{~g}^{-1}$ for the MOF powder - as measured by mercury intrusion. It should be noted that MIL-101 generally has a way higher specific surface area - even exceeding $5000 \mathrm{~m}^{2} \mathrm{~g}^{-1}$ - when assessed by $\mathrm{N}_{2}$ physisorption. In line with the mercury intrusion measurements, the $\mathrm{CO}_{2}$ adsorption capacity of the MIL-101 extrudates and powder reached 1.5 and $1.9 \mathrm{mmol} \mathrm{g}^{-1}$ at 4.5 bar and $25{ }^{\circ} \mathrm{C}$, respectively.

Recently, Hong et al. ${ }^{81}$ published a comparative study to assess the $\mathrm{CO}_{2}$ adsorption capacities of MIL-101 and 13X zeolite extrudates, the benchmark adsorbent. The extrusion process was nearly the same as described in their previous work with a slight modification of the paste composition to lower the binder content to $25 \mathrm{wt} \%$ (against $40 \mathrm{wt} \%$ ) and the drying step to $5{ }^{\circ} \mathrm{C}$ (against $10{ }^{\circ} \mathrm{C}$ ), while firing was increased to $205{ }^{\circ} \mathrm{C}$ 

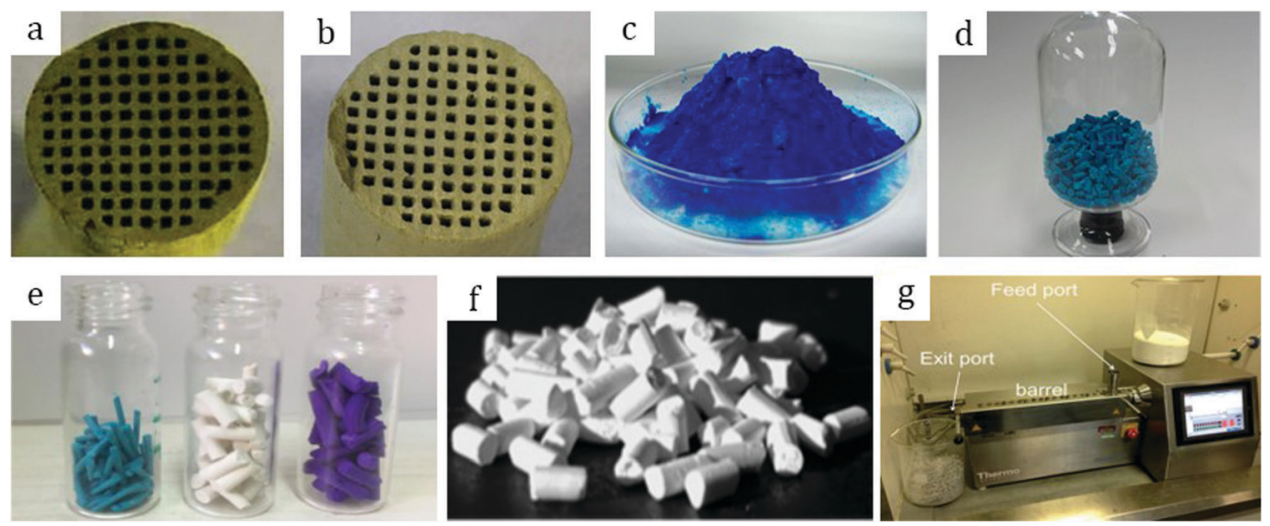

Fig. 7 Photograph of MIL-101 extrudates (a) of $7 \mathrm{~cm}$ long cylinders with a channel size of $0.9 \mathrm{~mm}$ made by Hong et al. ${ }^{80}$ Copyright 2015 Elsevier. Photograph of MIL-101 extrudates (b) of $10 \mathrm{~cm}$ long cylinders with a channel size of 0.9 made by Hong et al. ${ }^{81}$ Copyright 2020 Elsevier. As-synthesized HKUST-1 (c) and its extruded form (d) prepared by Kim et al. ${ }^{48}$ Copyright 2012 Elsevier. HKUST-1 (left), ZIF-8 (middle), and UTSA-16 (right) extrudates (e) prepared by Delgado et al. ${ }^{84}$ Copyright 2017 Elsevier. MIL-53 extrudates (f) prepared by Kriesten et al. ${ }^{87}$ Copyright 2019 Wiley. A twin screw extruder (g) used by Crawford et al. ${ }^{92}$ Copyright 2015 The Royal Society of Chemistry.

(against $\left.150{ }^{\circ} \mathrm{C}\right)($ Fig. $7 \mathrm{~b})$. The thus-prepared extrudates exhibited a specific surface area, as assessed by $\mathrm{Hg}$ intrusion, of $195 \mathrm{~m}^{2} \mathrm{~g}^{-1}$, comparable to the MIL-101 powder. As a result, the MIL-101 extrudates demonstrated a $\mathrm{CO}_{2}$ breakthrough adsorption capacity of $1.11 \mathrm{mmol} \mathrm{g}^{-1}$, higher than that of $13 \mathrm{X}$ zeolite extrudates $\left(0.81 \mathrm{mmol} \mathrm{g}^{-1}\right)$ under 2 bar (absolute pressure) of $40 \mathrm{vol} \% \mathrm{CO}_{2}$ at room temperature.

\section{Manual extrusion}

In some cases, the extrusion process can be reproduced using laboratory or home-made equipment. Thus, Kim et al. ${ }^{48}$ reported the manual fabrication of $\mathrm{Cu}_{3}(\mathrm{BTC})_{2}$ (HKUST-1) extrudates. Typically, they first formed a paste by mixing the parent MOF with the binder, a $15 \mathrm{wt} \%$ solution of polyvinyl alcohol in water. Thereafter, the paste was transferred to a home-made extruder, and the as-produced extrudates were subsequently cut and dried at $120^{\circ} \mathrm{C}$ for $12 \mathrm{~h}$ under vacuum (Fig. $7 \mathrm{c}$ and d). Upon extrusion, the crystal structure of HKUST-1 was preserved as confirmed by the XRD patterns. However, there was a considerable decrease in specific surface area associated with shaping, from 1737 to $963 \mathrm{~m}^{2} \mathrm{~g}^{-1}$. The total loss, around 45\%, indicates partial pore blocking. Similar results were reported by Majano and Pérez-Ramírez ${ }^{79}$ when using $20 \mathrm{wt} \%$ kaolin as a binder.

The extrudates were further applied for $\mathrm{CO}_{2}$ and water-vapor adsorptions. The latter revealed identical isotherm shapes for both the powder and extrudates. This suggests that the intrinsic hydrophilicity/hydrophobicity balance of HKUST-1 was not altered upon shaping. Besides, $\mathrm{CO}_{2}$ adsorption experiments at $25{ }^{\circ} \mathrm{C}$ revealed gravimetric uptakes of 218 and $129 \mathrm{mg} \mathrm{g}^{-1}$ for the MOF in powder and extruded forms, respectively. The decrease of $\mathrm{CO}_{2}$ uptake demonstrated by the latter is in agreement with the loss in specific surface area upon extrusion.

In 2014 , Grande et al. ${ }^{82}$ performed a study on the manual extrusion of Co-based UTSA-16 with emphasis on the paste composition. To form the paste, they combined polyvinyl alcohol as the binder and a water/propanol (1/1) mixture as the plasticizer. The paste was further extruded into strips using a syringe of a chosen diameter. The thus-shaped MOFs were then dried at $80^{\circ} \mathrm{C}$ for $12 \mathrm{~h}$. When varying the binder content, no significant loss in specific surface area with $2 \mathrm{wt} \%$ binder was observed. A further increase to $3 \mathrm{wt} \%$ PVA led to a $5 \%$ loss of SSA. Notably, the authors stated that an activation temperature lower than $120{ }^{\circ} \mathrm{C}$ was insufficient to remove the water/ propanol mixture. At the same time, $2 \mathrm{wt} \%$ binder was found to be adequate to provide a decent crushing strength of around $20 \mathrm{~N}$ upon conventional compression tests, comparable to commercial zeolite $4 \mathrm{~A}$ extrudates $(12 \mathrm{~N})$. For comparison, the absence of a binder resulted in a lower mechanical strength of around $7 \mathrm{~N}$.

The adsorptive capacities of these UTSA-16 extrudates towards model gases, and especially $\mathrm{CO}_{2}$, were evaluated in a following study by Agueda et al. ${ }^{83}$ As compared to their powder counterpart, the UTSA-16 extrudates, exhibiting a BET surface area of $805 \mathrm{~m}^{2} \mathrm{~g}^{-1}$, demonstrated a $15 \%$ loss of $\mathrm{CO}_{2}$ uptake at $25{ }^{\circ} \mathrm{C}$. This was attributed to the partial pore blocking caused by the binder presence. Still, the $\mathrm{CO}_{2}$ uptake of the extrudates is in the same range ( $4.0 \mathrm{~mol} \mathrm{~L}^{-1}$ at $\left.1 \mathrm{bar}\right)$ as those of commercial adsorbents based on activated carbon and zeolite 13X: 1.5 and $5.5 \mathrm{~mol} \mathrm{~L}^{-1}$ at 1 bar, respectively.

Following UTSA-16, Delgado et al. ${ }^{84}$ studied the extrusion and resulting adsorptive properties of HKUST-1 and ZIF-8 extrudates (Fig. 7e). Their textural properties as measured by $\mathrm{N}_{2}$ physisorption revealed a moderate decrease in surface area when shaped with $2.9 \mathrm{wt} \%$ binder, from $1700 \mathrm{~m}^{2} \mathrm{~g}^{-1}$ (HKUST-1 powder) to $1610 \mathrm{~m}^{2} \mathrm{~g}^{-1}$ (HKUST-1 extrudates, $\sim 5 \%$ loss). The loss was even reduced to $2 \%$ for ZIF- 8 , from 1680 to $1640 \mathrm{~m}^{2} \mathrm{~g}^{-1}$. This indicates a negligible pore blocking effect caused by the binder, as reported by Grande $e t$ al. ${ }^{82}$ as well as excellent structural stability upon manual extrusion - while HKUST-1 experienced a more pronounced textural and structural degradation using piston and single-screw extruders. As expected, the ZIF-8 extrudates demonstrated lower $\mathrm{H}_{2}$ and $\mathrm{CO}_{2}$ uptakes as compared to HKUST- 1 extrudates in a temperature range of $25-65{ }^{\circ} \mathrm{C}$ and pressures up to $50 \mathrm{bar}$, due to the lower 
affinity of gases with the ZIF-8 surface. As a link to the previous studies, heats of $\mathrm{CO}_{2}$ adsorption for the given MOFs as well as for the UTSA-16 extrudates were provided. The latter showed the highest value of released heat $\left(38 \mathrm{~kJ} \mathrm{~mol}^{-1}\right)$ at $20 \mathrm{bar}$ and $25{ }^{\circ} \mathrm{C}$, twice as much as those of the HKUST- 1 and ZIF- 8 extrudates. This indicates a considerable affinity of the UTSA-16 surface towards $\mathrm{CO}_{2}$, making it a promising candidate for $\mathrm{CO}_{2}$ capture.

Another study on manual extrusion was done by $\mathrm{Pu}$ et al. ${ }^{85}$ They investigated the shaping of Mg-based MOF-74/CPO-27 with KH570 (3-(trimethoxysilyl)propyl methacrylate) as a binder. For a typical extrusion procedure, they mixed a pre-defined amount of the parent MOF with a KH570 methanolic solution to form a paste. It was then extruded through a $1 \mathrm{~mL}$ injection syringe to form strips which were subsequently dried and cut into the final extrudates with dimensions of $1.5 \mathrm{~mm}$ length and $2 \mathrm{~mm}$ diameter. This procedure had no visible effect on the structure of the extruded MOF crystals as observed by XRD. Moreover, the thus-shaped MOF-74 exhibited a SSA of $1170 \mathrm{~m}^{2} \mathrm{~g}^{-1}$, as compared to $1230 \mathrm{~m}^{2} \mathrm{~g}^{-1}$ ( $\sim 5 \%$ drop) shown by its powder form as measured by the BET method with $\mathrm{CO}_{2}$ as the adsorbate. The extrudates presented a slightly preferential uptake of ethylene $\left(6.06 \mathrm{mmol} \mathrm{g}^{-1}\right)$ over ethane $\left(5.49 \mathrm{mmol} \mathrm{g}^{-1}\right)$ at $25{ }^{\circ} \mathrm{C}$ and $1 \mathrm{bar}$, as in the case of its powder counterpart (7.37 and $6.61 \mathrm{mmol} \mathrm{g}^{-1}$, respectively). Of note, the deterioration of adsorptive properties upon extrusion exceeds 5\% and might thus indicate partial pore blocking. Comparatively, zeolite $13 \mathrm{X}$, one of the commercial adsorbents, demonstrates ethylene and ethane uptakes of 3.32 and $2.70 \mathrm{mmol} \mathrm{g}^{-1}$, respectively, under the same conditions.

The study by Cousin-Saint-Remi et al. ${ }^{86}$ addressed the careful choice of binder for the preparation of mechanically stable extrudates from ZIF-8 powder, a MOF demonstrating relative stability upon more constraining processes such as pelletization (Table 3). Within the scope of their study, a series of binders were tested but only a few yielded robust objects. Thus, when shaped with typical inorganic binders such as silica, bentonite or kaolinite clays, the final objects broke apart upon extrusion. Methylcellulose and polystyrene were not appropriate either, due to the targeted application - COV adsorption. Therefore, a few polymeric binding agents, namely polyvinyl chloride (PVC), polyvinyl formal (PVF) and polyetherimide (PEI), yielded appropriate extrudates. For a typical formulation procedure, $1 \mathrm{~g}$ of the parent MOF was mixed with 7.5-30 wt\% binder followed by the gradual addition of $10 \mathrm{~mL}$ of solvent (DMF, DMSO or DMA). The thus-formed paste was extruded through the $2 \mathrm{~mm}$-tip of a syringe and subsequently dried overnight at room temperature. These extrudates were further applied to ethanol vapor adsorption at $50{ }^{\circ} \mathrm{C}$, and the results suggested a slight decrease in total gravimetric capacity at 127.5 mbar for the ZIF-8 extrudates compared to their parent powder, from 0.28 to $0.24 \mathrm{~g} \mathrm{~g}^{-1}(-15 \%)$. This decrease agrees with the total content of binder, suggesting a negligible pore blocking effect caused by its presence. Furthermore, the breakthrough experiments done with a mixture of acetone, ethanol and 1butanol diluted in water demonstrated the preferential adsorption of the latter on ZIF-8. Notably, the extrudates exhibited a broadening of elution profiles compared to the powder, indicating additional diffusion resistance created by the binder.

Extrusion of an Al-based MIL-53 MOF was studied by Kriesten et $a l{ }^{87}$ They investigated more specifically the framework "breathing" properties of MIL-53 and their evolution upon shaping. For the shaping procedure, they mixed a predefined amount of the parent MOF with 2-10 wt\% methyl cellulose (MC) as a binding agent followed by the dropwise addition of a $50 / 50$ vol\% water/ethanol mixture. Upon mixing, the thusformed homogeneous paste was further extruded through the $5 \mathrm{~mm}$-tip of a syringe to draw strips which were dried at room temperature, and finally cut into extrudates with lengths of 1.8 to $4.4 \mathrm{~mm}$ and diameters of 1.33 to $2.22 \mathrm{~mm}$ (Fig. 7f). As confirmed by XRD, the crystallinity of MIL-53 was preserved upon shaping for all the extrudates with a binder content of up to $10 \mathrm{wt} \%$. Furthermore, they retained the majority of their intrinsic textural properties. Thus, the parent MIL-53 exhibited a $S_{\mathrm{BET}}$ of $1525 \mathrm{~m}^{2} \mathrm{~g}^{-1}$, while the extrudates with 2, 5 and $10 \mathrm{wt} \%$ MC showed $S_{\text {BET }}$ of 1405,1280 and $1158 \mathrm{~m}^{2} \mathrm{~g}^{-1}$ corresponding to $5.9,11.6$ and $15.5 \%$ decreases, respectively. The difference might indicate a slight pore blocking effect caused by the binder. Notably, a second type of MC binder with higher molecular weight yielded a comparable decrease in BET surface area.

Interestingly, the framework "breathing" property of MIL-53 was shown to be preserved upon shaping by in situ XRD under humid conditions. Indeed, the phase transition from large to narrow pores was demonstrated to take place over the $20-45 \%$ relative humidity $(\mathrm{RH})$ range. Higher $\mathrm{RH}$ resulted in a faster phase transition. It was thus evident that the binder had no hindrance to the transition, and in fact, there was no relation between its content and the kinetics of the phase transition. Moreover, this breathing property was also observed upon $\mathrm{CO}_{2}$ adsorption at $30{ }^{\circ} \mathrm{C}$. As with $\mathrm{N}_{2}$, there was only a slight decrease of total $\mathrm{CO}_{2}$ uptake ( $\left.\sim 4 \%\right)$ upon adsorption on MIL-53 extrudates formed with $3 \mathrm{wt} \%$ binder as compared to the powder. Furthermore, the shape of the isotherm was preserved, revealing the breathing phenomenon resulting in an isotherm with a hysteresis loop.

\section{Reactive extrusion}

Reactive extrusion, related to mechanochemical synthesis, is generally based on reactions between solid precursors with little to no solvent added. The energy needed to initiate the reactions is classically provided by frictions and shocks as found in milling or grinding processes. The main advantage of the mechanochemical approach for MOF synthesis is the minimization or complete avoidance of the use of solvents which are often hazardous liquids. Up to now, this approach has been applied to many MOFs among which are HKUST- $1,{ }^{88}$ ZIF-8, ${ }^{89}$ UiO-67, ${ }^{90}$ and MOF-5. ${ }^{91}$ Although mechanochemistry allows faster syntheses as compared to classical solventassisted syntheses, its up-scaling remains an issue.

In 2015, Crawford et al. ${ }^{92}$ described the mechanochemical synthesis of MOFs using a twin screw extruder (TSE) (Fig. 7g), thus combining synthesis and shaping in one step. Indeed, the 
rotating screws composed of different zones (conveying, shearing, kneading) displace the starting solid MOF precursors along the heated barrel with good control over the residence time, and the mixing duration and intensity. Hence, through the combination of shearing and compression forces, solid-state reactions between the precursors can be obtained. Ideally, upon reaching the exit port, the product is formed and it is further drawn through a die into extrudates. Of note, the controllable heating of the barrel allows better control over the reaction conditions as compared to conventional milling approaches.

For instance, the authors used copper hydroxide and trimesic acid mixed with methanol as a feed material to produce HKUST-1. Upon extrusion at room temperature, the product was washed with ethanol and dried to yield HKUST-1 extrudates with a specific surface area of $1738 \mathrm{~m}^{2} \mathrm{~g}^{-1}$ and a crystal structure expected for this MOF. Furthermore, the authors showed that ZIF-8 extrudates can be made by both single and twin screw extrusion processes. For this, they used a blend of zinc carbonate and 2-methylimidazole with no solvent added and extruded it at $200{ }^{\circ} \mathrm{C}$. In both cases, the processes yielded a crystalline product with the ZIF-8 topology and high surface areas: $1604 \mathrm{~m}^{2} \mathrm{~g}^{-1}$ (twin screw) and $1750 \mathrm{~m}^{2} \mathrm{~g}^{-1}$ (single screw). Lastly, the authors produced a highly crystalline Al-fumarate with a BET surface area of $1010 \mathrm{~m}^{2} \mathrm{~g}^{-1}$ by extruding a mixture of Al-sulfate, fumaric acid and sodium hydroxide at $150{ }^{\circ} \mathrm{C}$. It is worth noting that this approach enables the production of MOFs with decent space-time yields (STY) as single and twin screw extrusions are continuous processes.

To conclude, extrusion is another widely used technique to process MOF powders into different shapes. Compaction of the individual MOF particles is driven by the difference of diameter between upstream and downstream while keeping constant the flowrate. For flowability to be obtained, the MOF powders are typically mixed with a binder and a plasticizer, with the latter enabling the formation of a rheofluidifying paste. Since the binder cannot be removed from a shaped MOF object, certain care should be given to fine-tuning the binder content in order to avoid critical pore blocking. Generally, extruders with different forms and numbers of screws allow the formation of hollow or dense objects with different shapes and sizes. This advantage makes extrusion a preferred technique over pelletization. In addition, manual extrusion of a paste through a syringe is also possible. Comparative data are given in Fig. 8 for two wellstudied MOFs: HKUST-1 and ZIF-8.

A positive effect of extrusion is evident from Fig. 8 as in the case of ZIF-8; the loss in surface area upon extrusion is negligible $(-2 \%)$ and coherent with the amount of added binder, while upon pelletization the loss in surface area is more pronounced $(-13 \%)$. At the same time, for HKUST-1 the decrease in surface area is somewhat comparable upon pelletization and extrusion. However, the latter is made with $20 \mathrm{wt} \%$ binder, which causes drastic pore blocking, while the loss in surface area upon pelletization is due to the considerable framework collapse.

\section{D printing}

3D printing (also known as "additive manufacturing" or "robocasting") has attracted significant interest as a shaping tool over the past decade. Its recent applications have expanded beyond the fabrication of thermoplastic composites for daily use to find unique applications in medicine, architecture and many other fields. ${ }^{93}$ As with other shaping techniques in materials science, 3D printing enables the assembly of parent powders into solid 3D objects of various shapes and sizes. It specifically allows the manufacture of structured adsorbents or catalysts for large industrial reactors. For MOFs, the most common technology applied is direct ink writing (DIW) ${ }^{94}$ The basic principle of this method resembles the conventional extrusion process and is composed of similar steps. Namely, the first step implies the formation of a paste by suspending the parent powder in a liquid (water or short-chain alcohols).
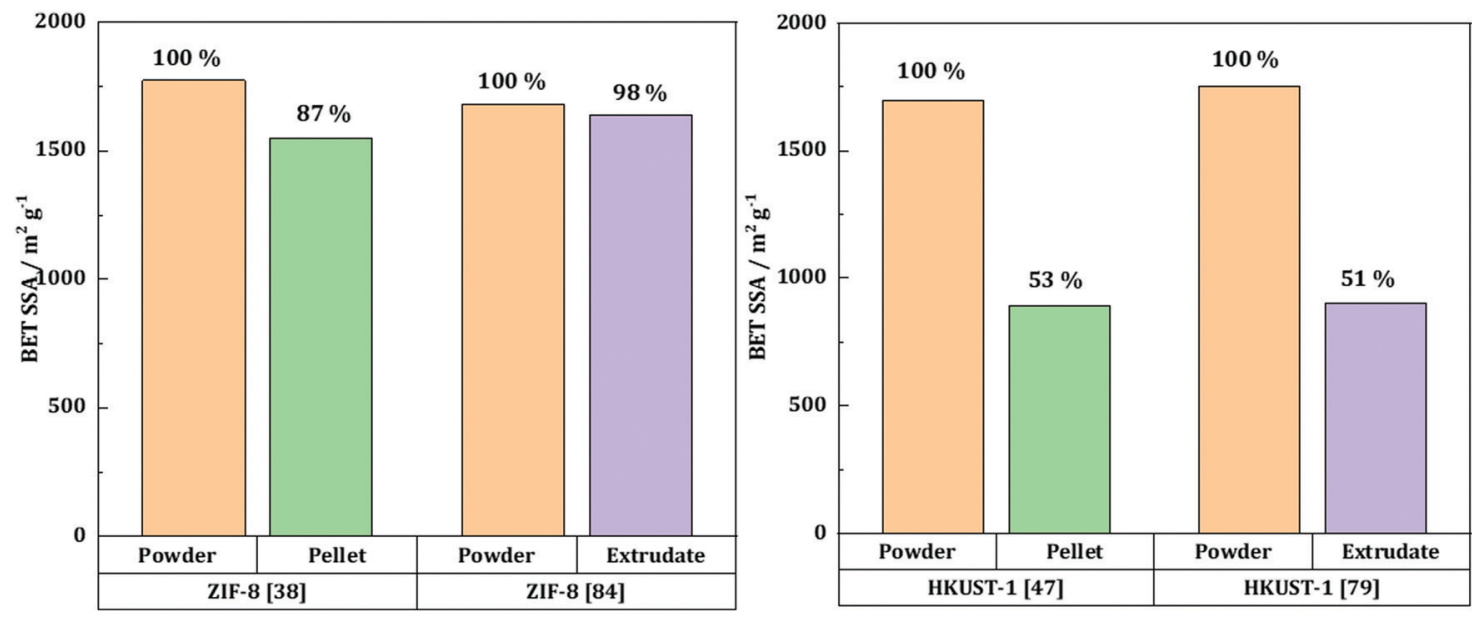

Fig. 8 Comparison of the extruded ${ }^{84}$ and pelletized ${ }^{38}$ ZIF-8 (left); and the pelletized ${ }^{47}$ and extruded ${ }^{79}$ HKUST-1 (right). Numbers indicate the BET SSA upon shaping the pristine powder into extrudates and pellets. 


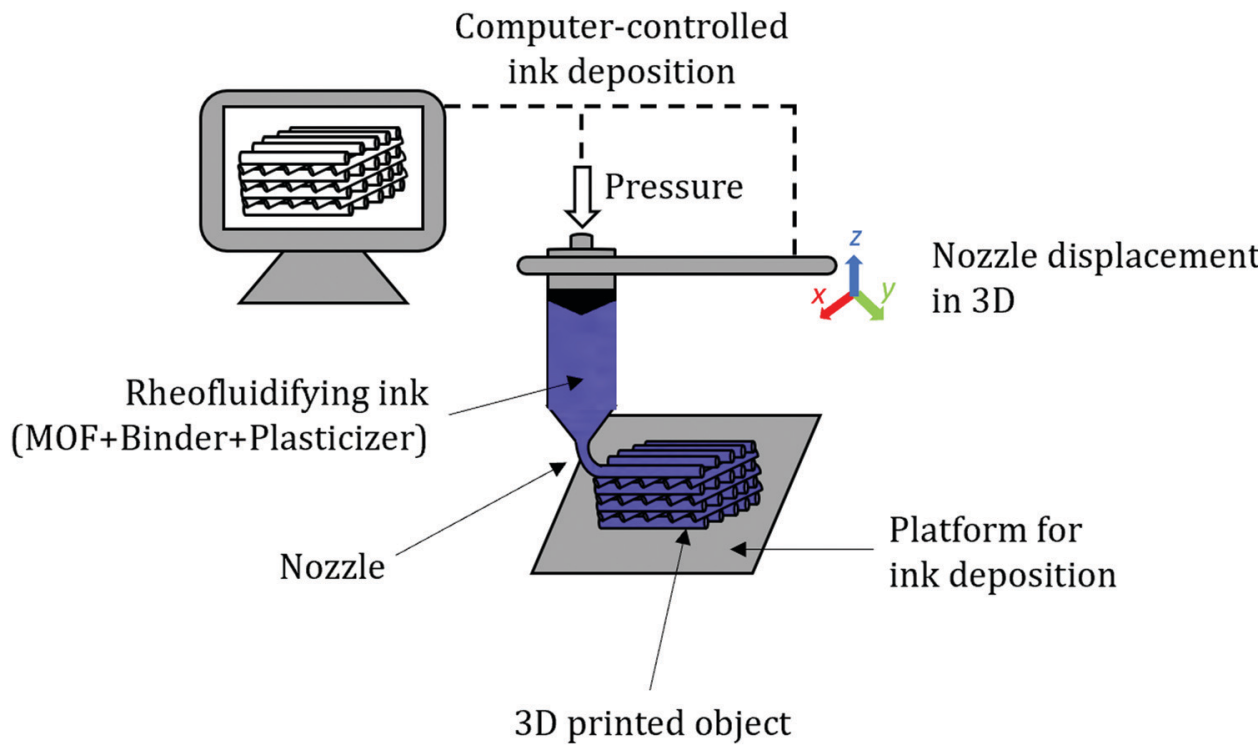

Fig. 9 Schematic representation of the 3D printing process via the Direct Ink Writing (DIW) method.

Thereafter, the paste is pushed through a thin printing nozzle and subsequently deposited on a substrate in a controllable layer-by-layer manner designed using a slicing software package. Upon building up the desired object with a pre-defined geometry and dimensions, it is further subjected to a thermal treatment for the removal of the liquid constituents. Apart from DIW, other approaches include Digital Light Processing (DLP) and Selective Laser Sintering (SLS). The former employs UV irradiation to locally cure a resin containing a monomer and the parent powder. In turn, SLS implies the melting of a thermoplastic material with a laser beam in the presence of the parent powder.

A typical 3D printer for DIW consists of a substrate, a nozzle (syringe or extrusion head) and a series of interconnected vertical and horizontal bars which enable the displacement of the nozzle along the $x, y$, and $z$ axes (Fig. 9). The substrate is also mobile and often responsible for the movement along the $x$ and $y$ axes.

As in the case of extrusion, the paste formulation is a crucial step in 3D printing and should yield a final composition with appropriate rheological properties. Apart from the parent powder and a liquid, the paste is also composed of a binder and a plasticizer. The former provides adequate mechanical resistance to the final 3D objects, while the latter improves the flowability and plasticity of the paste to be printed. One of the major differences is the printing nozzle: while the die in extruders can reach sizes up to a few centimeters, in 3D printers the nozzle (or needle) is typically smaller than millimeters in diameter. Such a thin nozzle allows designing objects with complex geometries that would be challenging to obtain via a conventional method.

Recently, 3D printing has been applied to a large number of structured adsorbents and catalysts. Thus, $\mathrm{Al}_{2} \mathrm{O}_{3}$ was shown to be printable into monoliths exhibiting high catalytic efficiency as well as good recyclability. ${ }^{95}$ Zeolites $13 \mathrm{X}$ and $5 \mathrm{~A}$ have also been printed into monoliths for $\mathrm{CO}_{2}$ removal purposes, ${ }^{96}$ while a 3D-printed zeolite (ZSM-5) has been probed for $\mathrm{CO}_{2}, \mathrm{CH}_{4}$ and $\mathrm{N}_{2}$ separation. Among the other printed structures can be found carbons, ${ }^{97}$ amorphous aluminosilicates ${ }^{98}$ and other classes of adsorbents. ${ }^{99}$

3D printed MOFs have become a frequently discussed topic in the literature. Their excellent textural properties make them promising materials for gas adsorption, storage and purification purposes. These include the most studied representatives such as HKUST-1, ZIF-8 and UiO-66 (Table 11).

As is evident from Table 11, different binder/plasticizer systems have been probed, as well as a binderless approach. In some cases, the mechanical stability of the printed objects is provided by polymer matrices on which MOFs are deposited upon printing.

\section{MOF growth on pre-printed matrices}

The earliest studies on 3D printing include the surface coating of a thermoplastic polymeric matrix, acrylonitrile butadiene styrene (ABS), with a Cu-based HKUST-1 MOF by Wang et al. ${ }^{100}$ The authors first immersed the printed ABS objects in a solution containing the $\mathrm{Cu}$-precursor, followed by immersion in a solution containing the organic ligand. Eventually, the objects were dried and washed prior to further immersion. The procedure was repeated up to 8 cycles (Fig. 10a). Upon the final drying step, the HKUST-1/ABS composites were shown to exhibit reflections characteristic of HKUST-1, revealing its successful crystallization on the ABS surface. Moreover, the composite was able to remove methylene blue from an aqueous solution, due to the $\mathrm{Cu}^{2+}$ sites present in the MOF.

Following a comparable method, Zhang et al. deposited up to eight layers of HKUST-1 on PLA films. ${ }^{101}$ After the eighth cycle and further activation, a dark blue film was obtained, presenting the main diffraction peaks of HKUST-1 in its XRD pattern. Moreover, crystals with an octahedral shape are clearly 
Table 11 List of MOFs formulated via 3D printing

\begin{tabular}{|c|c|c|c|c|c|c|}
\hline \multirow[b]{2}{*}{ MOF } & \multirow[b]{2}{*}{ Metal/ligand } & \multirow[b]{2}{*}{ Binder, wt $\%$} & \multirow[b]{2}{*}{ Plasticizer } & \multicolumn{2}{|c|}{$S_{\mathrm{BET}}, \mathrm{m}^{2} \mathrm{~g}^{-1}$} & \multirow[b]{2}{*}{ Ref. } \\
\hline & & & & Powder & 3D object & \\
\hline \multicolumn{7}{|c|}{ MOF growth on pre-printed matrices } \\
\hline HKUST-1 & $\mathrm{Cu} / \mathrm{BTC}$ & ABS & None & - & - & 100 \\
\hline HKUST-1 & $\mathrm{Cu} / \mathrm{BTC}$ & PLA & None & - & - & 101 \\
\hline HKUST-1 & $\mathrm{Cu} / \mathrm{BTC}$ & $\mathrm{SiO}_{2}$ & None & - & 223 & 103 \\
\hline UTSA-16 & $\mathrm{Co} / \mathrm{CA}$ & Kaolin, 10 & MC & 630 & $620(-2 \%)$ & 102 \\
\hline \multirow[t]{2}{*}{ MIL-100 } & $\mathrm{Fe} / \mathrm{BTC}$ & $\mathrm{SiO}_{2}$ & None & 一 & 82 & 103 \\
\hline & & & & \multicolumn{2}{|c|}{$S_{\mathrm{BET}}, \mathrm{m}^{2} \mathrm{~g}^{-1}$} & \\
\hline MOF & Metal/ligand & Matrix, wt\% & Plasticizer, wt\% & Powder & 3D object & Ref. \\
\hline \multicolumn{7}{|c|}{ Direct writing of MOF-based inks (DIW) within polymer matrices } \\
\hline MOF-5 & $\mathrm{Zn} / \mathrm{BDC}$ & ABS, 90 & None & - & - & 104 \\
\hline HKUST-1 & $\mathrm{Cu} / \mathrm{BTC}$ & ABS, 90 & None & 776 & $329(-58 \%)$ & 105 \\
\hline ZIF-8 & $\mathrm{Zn} / \mathrm{MIM}$ & ABS, 90 & None & 1446 & $436(-70 \%)$ & 105 \\
\hline ZIF-8 & $\mathrm{Zn} / \mathrm{MIM}$ & PLA, 60 & None & 1752 & $531(-70 \%)$ & 106 \\
\hline ZIF-8 & $\mathrm{Zn} / \mathrm{MIM}$ & TPU, 40 & PVDF-HFP, 20 & 1752 & $706(-60 \%)$ & 106 \\
\hline UiO-66 & $\mathrm{Zr} / \mathrm{BDC}$ & TPU, 40 & PVDF-HFP, 20 & 1144 & $303(-74 \%)$ & 106 \\
\hline
\end{tabular}

\begin{tabular}{|c|c|c|c|c|c|c|}
\hline \multirow[b]{2}{*}{ MOF } & \multirow[b]{2}{*}{ Metal/ligand } & \multirow[b]{2}{*}{ Binder, wt\% } & \multirow[b]{2}{*}{ Plasticizer } & \multicolumn{2}{|c|}{$\underline{S_{\mathrm{BET}}, \mathrm{m}^{2} \mathrm{~g}^{-1}}$} & \multirow[b]{2}{*}{ Ref } \\
\hline & & & & Powder & 3D object & \\
\hline \multicolumn{7}{|c|}{ Direct writing of MOF-based inks (DIW) } \\
\hline UTSA-16 & $\mathrm{Co} / \mathrm{CA}$ & Bentonite, 10 & PVA & 631 & $444(-30 \%)$ & 107 \\
\hline UTSA-16 & $\mathrm{Co} / \mathrm{CA}$ & Boehmite, 31 & HPC & - & 540 & 118 \\
\hline $\mathrm{Ni}(\mathrm{bdc})(\text { ted })_{0.5}$ & Ni/BDC, TED & PVA, 20 & EtOH:DMSO & 1802 & $1325(-27 \%)$ & 108 \\
\hline CPL-1 & $\mathrm{Cu} / \mathrm{PZDC}, \mathrm{PYZ}$ & HEC, 6.7 & PVA & 198 & $68(-66 \%)$ & 115 \\
\hline $\mathrm{UiO}-66-\mathrm{NH}_{2}$ & $\mathrm{Zr} / 2$-aminoBDC & HEC, 7.3 & PVA & 1123 & $871(-22 \%)$ & 115 \\
\hline HKUST-1 & $\mathrm{Cu} / \mathrm{BTC}$ & HEC, 19.5 & PVA & 1581 & $1181(-25 \%)$ & 115 \\
\hline HKUST-1 & $\mathrm{Cu} / \mathrm{BTC}$ & SA & Gelatin & 1563 & $83(-95 \%)$ & 116 \\
\hline HKUST-1 & $\mathrm{Cu} / \mathrm{BTC}$ & None & EtOH & 1850 & $1134(-39 \%)$ & 117 \\
\hline HKUST-1 & $\mathrm{Cu} / \mathrm{BTC}$ & Bentonite, 21 & MC:PVA & 830 & $500(-40 \%)$ & 111 \\
\hline HKUST-1 & $\mathrm{Cu} / \mathrm{BTC}$ & Bentonite, 15 & PVA & 830 & $470(-43 \%)$ & 111 \\
\hline HKUST-1 & $\mathrm{Cu} / \mathrm{BTC}$ & Torlon, 37.5 & PVP & - & 290 & 110 \\
\hline MOF-74 & $\mathrm{Ni} / 2,5-(\mathrm{OH})_{2} \mathrm{BDC}$ & Torlon, 52.9 & PVP & - & 80 & 110 \\
\hline MOF-74 & $\mathrm{Ni} / 2,5-(\mathrm{OH})_{2} \mathrm{BDC}$ & Bentonite, 15 & PVA & 1180 & $737(-38 \%)$ & 107 \\
\hline ZIF-7 & $\mathrm{Zn} / \mathrm{BIM}$ & Silica, 15 & $\mathrm{H}_{2} \mathrm{O}: \mathrm{MeOH}$ & 16 & 40 & 108 \\
\hline ZIF-8 & $\mathrm{Zn} / \mathrm{MIM}$ & Bentonite, 16.7 & $\mathrm{MC}$ & 1415 & $1070(-24 \%)$ & 112 \\
\hline ZIF-8 & $\mathrm{Zn} / \mathrm{MIM}$ & HEC, 13.2 & PVA & 1711 & $1690(-1 \%)$ & 115 \\
\hline ZIF-8 & $\mathrm{Zn} / \mathrm{MIM}$ & SA & TOCNF & - & 900 & 119 \\
\hline MIL-100 & $\mathrm{Fe} / \mathrm{BTC}$ & SA & TOCNF & - & - & 119 \\
\hline MIL-101 & $\mathrm{Cr} / \mathrm{BDC}$ & Bentonite, 15 & PVA & 2400 & $2200(-8 \%)$ & 109 \\
\hline
\end{tabular}

\begin{tabular}{|c|c|c|c|c|c|c|}
\hline \multirow[b]{2}{*}{ MOF } & \multirow[b]{2}{*}{ Metal/ligand } & \multirow[b]{2}{*}{ Matrix, wt\% } & \multirow[b]{2}{*}{ Photoinitiator } & \multicolumn{2}{|c|}{$S_{\mathrm{BET}}, \mathrm{m}^{2} \mathrm{~g}^{-1}$} & \multirow[b]{2}{*}{ Ref } \\
\hline & & & & Powder & 3D object & \\
\hline \multicolumn{7}{|c|}{ UV irradiation-assisted 3D printing, digital light processing (DLP) } \\
\hline UiO-66 & $\mathrm{Zr} / \mathrm{BDC}$ & TMPPTA, 44 & HMPP, PPO & 1590 & $633(-60 \%)$ & 120 \\
\hline HKUST-1 & $\mathrm{Cu} / \mathrm{BTC}$ & PEA/PGD & I-819, I-184 & - & - & 121 \\
\hline \multirow[t]{2}{*}{ HKUST-1 } & $\mathrm{Cu} / \mathrm{BTC}$ & AAm, 25 & $\mathrm{I}-2959$ & - & - & 122 \\
\hline & & & & \multicolumn{2}{|c|}{$\underline{S_{\mathrm{BET}}, \mathrm{m}^{2} \mathrm{~g}^{-1}}$} & \\
\hline MOF & Metal/ligand & Matrix, wt $\%$ & Plasticizer & Powder & 3D object & Ref. \\
\hline \multicolumn{7}{|c|}{ Laser-assisted 3D printing, selective laser sintering (SLS) } \\
\hline $\mathrm{NH}_{2}$-MIL-101 & $\mathrm{Al} / 2$-aminoBDC & PA12 & None & - & - & 123 \\
\hline MOF-801 & $\mathrm{Zr} / \mathrm{FA}$ & PA12 & None & - & - & 123 \\
\hline HKUST-1 & $\mathrm{Cu} / \mathrm{BTC}$ & PA12 & None & - & - & 123 \\
\hline HKUST-1 & $\mathrm{Cu} / \mathrm{BTC}$ & PA12 & None & - & 40 & 124 \\
\hline ZIF-67 & $\mathrm{Co} / \mathrm{MIM}$ & PA12 & None & - & 640 & 123 \\
\hline ZIF-8 & $\mathrm{Zn} / \mathrm{MIM}$ & PA12 & None & 1359 & $332(-76 \%)$ & 123 \\
\hline
\end{tabular}

Ligand codes: BTC - benzene-1,3,5-tricarboxylic acid; CA - citric acid; BDC - benzene-1,4-dicarboxylic acid; MIM - 2-methyl imidazole; TED - 1,4biazabicyclo[2.2.2] octane; BiM - benzimidazole; PZDC - pyrazine-2,3-dicarboxylic acid; PYZ - pyrazine; and FA - fumaric acid. Binder/matrix codes: ABS acrylonitrile-butadiene-styrene; PLA - polylactic acid; TPU - thermoplastic polyurethane; PVA - polyvinyl alcohol; HEC - 2-hydroxyethyl cellulose; SA - sodium alginate; TMPPTA - trimethylolpropane propoxylate triacrylate; PEA - 2-phenoxyethyl acrylate; PGD - polyethylene glycol diacrylate; PA12 - polyamide 12; and AAm - acrylamide + N,N'-methylenebisacrylamide (0.06 wt\% acrylamide). Plasticizer codes: MC - methyl cellulose; HPC - hydroxypropylcellulose; DMSO - dimethyl sulfoxide; TOCNF - 2,2,6,6-tetramethylpiperidine-1-oxyl radical-mediated oxidized cellulose nanofibers; PVP - polyvinylpyrrolidone; and PVDF-HFP - poly(vinylidene fluoride-co-hexafluoropropylene). Photoinitiator codes: HMPP - 2-hydroxy-2-methylpropiophenone; PPO phenylbis(2,4,6-trimethylbenzoyl)phosphine oxide; I-189 - Irgacure-819; I-184 - Irgacure-184; and I-2959 - Irgacure-2959. “-” not specified. 

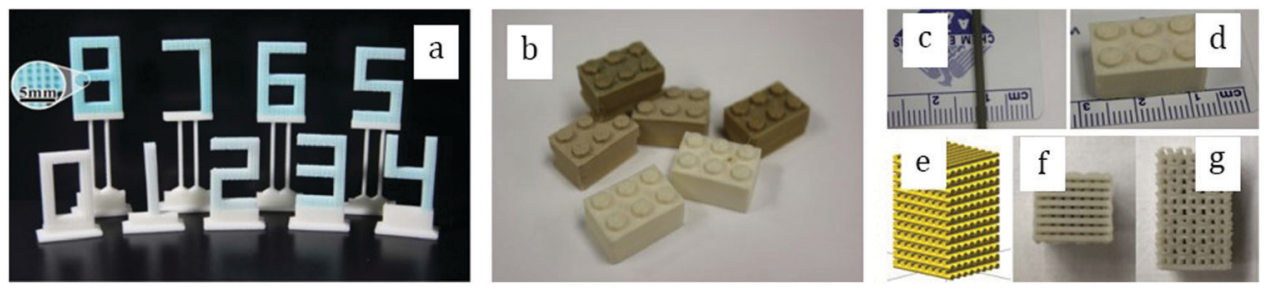

Fig. 10 Photographs of MOFs grown on pre-printed matrices: HKUST-1/ABS composites (a) printed in the form of numbers representing the number of cycles prepared by Wang et al. ${ }^{100}$ Copyright 2014 Springer Nature. 3D shapes made with $1 \%, 5 \%$, and $10 \%$ MOF-5 composites (b); filament used in the printing process (c); 3D object with a scale (d); image of the designed object (e); and top and side views of the 3D printed object ( $f$ and g) prepared by Kreider et al. ${ }^{104}$ Copyright 2017 Wiley.

visible in the SEM images. This functionalized film was applied for malachite green remediation in river water at a concentration of $10 \mathrm{mg} \mathrm{L}^{-1}$. After 10 minutes, $90 \%$ of the initial MG was adsorbed. The film was recycled up to 5 times, however with a certain decrease of efficiency over the cycles.

Another post-printing MOF growing method was applied by Lawson et al., ${ }^{102}$ who prepared UTSA-16/kaolin 3D printed composites. A paste was composed of a binder (kaolin), a plasticizer (methyl cellulose) and UTSA-16 precursors with adjustable loadings. Further, the paste was extruded into monoliths and subsequently exposed to solvothermal conditions to initiate crystal growth. This procedure enabled the obtention of UTSA-16/kaolin composites, with MOF loadings as high as $90 \mathrm{wt} \%$. According to XRD, the monoliths exhibited patterns matching with the pristine UTSA-16 powder. Besides, they exhibit pronounced textural properties with a $S_{\mathrm{BET}}$ of $620 \mathrm{~m}^{2} \mathrm{~g}^{-1}$, as high as that of their powder analogue $\left(630 \mathrm{~m}^{2} \mathrm{~g}^{-1}\right)$. These composites were shown to exhibit excellent $\mathrm{CO}_{2}$ separation properties over a mixture of $\mathrm{CH}_{4}, \mathrm{~N}_{2}$ and $\mathrm{H}_{2}$. Thus, the MOF-loaded monolith demonstrated $\mathrm{CO}_{2} / \mathrm{CH}_{4}$, $\mathrm{CO}_{2} / \mathrm{N}_{2}$ and $\mathrm{CO}_{2} / \mathrm{H}_{2}$ selectivities of 49,238 and 3725 , respectively. These values are comparable to those of the pristine UTSA-16 powder (52, 250, and 3750, respectively).

More recently, Zhou et al. ${ }^{103}$ deposited two MOFs, HKUST-1 and MIL-100(Fe), on the surfaces of silica grids printed following the direct-ink writing (DIW) approach and presenting two levels of macroporosity: large macropores of about $1 \mathrm{~mm}$, generated by 3D-printing a grid-like pattern, and smaller macropores of about $2 \mu \mathrm{m}$, due to the addition of polystyrene beads in the ink. After combustion of the beads and sintering at $900{ }^{\circ} \mathrm{C}$, fully macroporous solids were obtained (total pore volume $=0.01 \mathrm{~cm}^{3} \mathrm{~g}^{-1}$ as given by $\mathrm{N}_{2}$ physisorption). The grids were then surface-treated with $\mathrm{KOH}$ solution and coated with dopamine hydrochloride to improve the MOFs' adhesion. Finally, MIL-100(Fe) and HKUST-1 MOFs were grown at the surfaces of the macropores under solvothermal conditions. The obtained MOFs were highly crystalline and the crystal size was about $100 \mathrm{~nm}$. All bands identified by XPS and FT-IR spectroscopy were attributed to the MOF structures. Finally, these grids were attached to a 3D-printed plastic stirring device, and used for the Fenton-like catalytic degradation of a blend of dyes (methylene blue, rhodamine $\mathrm{B}$, malachite green and crystal violet, $20 \mathrm{mg} \mathrm{L}^{-1}$ each) from wastewater. $0.9 \mathrm{M} \mathrm{H}_{2} \mathrm{O}_{2}$ was added prior to each cycle, and up to 50 cycles were performed. Based on UV-visible spectrometry, after 20 minutes complete degradation of the dyes was observed with both MOFs at the end of the first cycle, while after 50 cycles the activity decreased to $75 \%$ and $52 \%$ using MIL-100(Fe) and HKUST-1, respectively.

Instead of depositing MOFs at the surfaces of pre-printed structures, another more direct approach would be to print MOF-polymer composite filaments. This pathway was first published by Kreider et al. ${ }^{104}$ who addressed the shaping of the Zn-based MOF-5 into monoliths via the use of a conventional thermoplastic 3D printer using $\mathrm{ABS}$ as the matrix. Although ABS is amongst the most used thermoplastic polymers for 3D printing, it only improves the mechanical stability of the printed objects by providing its own properties rather than by generating bonds with the individual MOF particles. Therefore, the amount of polymer should be adequately large to provide sufficient mechanical resistance to the final printed objects. Thus, the authors dissolved ABS in acetone with addition of a certain amount of pre-synthesized MOF- 5 (from 1 to $10 \mathrm{wt} \%$, on a dry basis). Upon solvent evaporation, the film was recovered and extruded into $1.75 \mathrm{~mm}$-wide filaments, which were subsequently 3D-printed via a nozzle heated at $230{ }^{\circ} \mathrm{C}$ onto a platform heated at $115{ }^{\circ} \mathrm{C}$ to yield objects of different shapes (Fig. 10b-g).

The thus-shaped objects were found to exhibit XRD patterns similar to a MOF-5 degraded by humidity. This was attributed to the formulation procedure prior to printing rather than to the printing procedure itself. Nevertheless, these 3D printed objects were shown to have the ability to adsorb $\mathrm{H}_{2}$ despite the complex polymer environment.

Nearly the same 3D printing process was applied by Bible et al. ${ }^{105}$ to prepare ZIF-8/ABS and HKUST-1/ABS composites with a MOF content of $10 \mathrm{wt} \%$. Interestingly, the composites exhibited reflections characteristic of the parent MOF powders, suggesting that the crystal structures were preserved upon the formulation procedure. Moreover, as said previously HKUST-1based materials tend to degrade upon exposure to a humid environment. However, the XRD pattern of a HKUST-1/ABS composite remained unchanged after keeping it in water for a week, suggesting that ABS, a hydrophobic material, prevents water from binding with $\mathrm{Cu}^{2+}$ ions and thus prevents significant hydrolysis. Both composites exhibited adsorptive features towards nitrogen with specific surface areas, $S_{\mathrm{BET}}$, of 436 and 
$329 \mathrm{~m}^{2} \mathrm{~g}^{-1}$ for ZIF-8/ABS and HKUST-1/ABS, respectively. Notably, the absence of a sharp increase in $\mathrm{N}_{2}$ uptake at low pressures, characteristic of microporous materials, was attributed to the slowed diffusion of $\mathrm{N}_{2}$ through the ABS-containing objects, and therefore incomplete micropore filling.

A similar methodology adopted by Evans et al. ${ }^{106}$ yielded rigid ZIF-8/PLA (polylactic acid) composites with MOF contents up to $40 \mathrm{wt} \%$. To achieve a homogeneous dispersion within the polymer matrix, the MOF powder was first suspended in ethyl acetate and mixed with PLA dissolved in $\mathrm{CH}_{3} \mathrm{Cl}$. The mixture was cast and left for drying before subsequent extrusion. Eventually, the thus-extruded filaments were used for 3Dprinting through a $400 \mu \mathrm{m}$ diameter nozzle preheated at $200{ }^{\circ} \mathrm{C}$. This protocol yielded 3D printed composites with XRD patterns presenting well-resolved reflections characteristic of ZIF-8, along with a $S_{\mathrm{BET}}$ of up to $531 \mathrm{~m}^{2} \mathrm{~g}^{-1}$, as compared to $1752 \mathrm{~m}^{2} \mathrm{~g}^{-1}$ for the original ZIF-8 powder (-70\%). This suggests blocking by few pores caused by PLA. The same shaping procedure was repeated with a thermoplastic polyurethane (TPU) polymer to yield flexible ZIF-8/TPU composites with the same $40 \mathrm{wt} \%$ MOF loading. Interestingly, a higher $S_{\mathrm{BET}}$ of $706 \mathrm{~m}^{2} \mathrm{~g}^{-1}$ was retained (-60\% compared to the powder), suggesting that the whole porosity remained accessible. Finally, the authors applied their methodology for the 3D-printing of another emblematic MOF, UiO-66, giving a composite material presenting a $S_{\text {BET }}$ of $303 \mathrm{~m}^{2} \mathrm{~g}^{-1}$.

\section{Direct writing of MOF-based inks (DIW)}

The first successful trial of direct ink writing using MOFs was performed by Thakkar et al., ${ }^{107}$ who shaped MOF-74 and UTSA16 powders into monoliths. For this purpose, they used bentonite clay as a binder and polyvinyl alcohol (PVA) as a plasticizer. Firstly, the paste formation step included mixing two solutions: the MOF powder and the binder in ethanol (1) and the PVA dissolved in water and ethanol (2). Upon mixing, the paste was transferred into a syringe and extruded through a $0.85 \mathrm{~mm}$ diameter nozzle in a layer-by-layer fashion, yielding monolith structures with uniform channels. Eventually, the printed objects with dimensions of $1.5 \mathrm{~cm}$ height $\times 1.5 \mathrm{~cm}$ diameter were dried at $100{ }^{\circ} \mathrm{C}$ to prevent cracks.

The thus-shaped MOFs were extensively characterized. The crystal structure was demonstrated by XRD to be preserved upon 3D printing. The slightly lower reflection intensities were attributed to the binder and plasticizer presence. In agreement with that, $\mathrm{N}_{2}$ physisorption measurements showed a consequent decrease in $S_{\mathrm{BET}}$ upon printing, from 1180 to $737 \mathrm{~m}^{2} \mathrm{~g}^{-1}$ for MOF-74 and from 631 to $444 \mathrm{~m}^{2} \mathrm{~g}^{-1}$ for UTSA-16. This suggests a decrease of 38 and $30 \%$ compared to their powder analogue, respectively. Interestingly, while the pore size distribution remained unchanged in the case of MOF-74, with the majority of pores in the range of 1-4 nm, the UTSA-16 monoliths were shown to possess mesopores $20-25 \mathrm{~nm}$ in size. This was attributed to the formation of straight channels as the result of interactions between the water present in the paste and the citrate units in the MOF framework. The cross-sectional area of the monoliths viewed via SEM revealed wall thicknesses/ channel widths of $0.4 / 0.7 \mathrm{~mm}$ and $0.8 / 1.1 \mathrm{~mm}$ for MOF-74 and UTSA-16, respectively. Interestingly, this alteration of the size originated from the different viscosities of the two pastes. Thus, the less viscous UTSA-16 paste expanded upon deposition and yielded thicker walls and larger channel sizes as compared to the more viscous MOF-74 paste (Fig. 11a). The MOF monoliths were further applied to $\mathrm{CO}_{2}$ adsorption measurements. Starting from a $0.5 \% \mathrm{CO}_{2} / \mathrm{N}_{2}$ gas mixture, the $\mathrm{CO}_{2}$ uptake capacities of MOF-74 and UTSA-16 3D-printed solids reached 1.35 and $1.31 \mathrm{mmol} \mathrm{g}^{-1}$, respectively. These values correspond to 79 and $87 \%$ of the $\mathrm{CO}_{2}$ uptake capacity of the corresponding MOF powders and agree with their total content in the monoliths.

A further study by Thakkar et $a .^{108}$ addressed the 3D printing of two MOFs, Ni-based Ni(bdc)(ted) $)_{0.5}$ and Zn-based ZIF-7, for ethane-selective adsorption. The paste was formed by mixing pre-synthesized $\mathrm{Ni}(\mathrm{bdc})(\mathrm{ted})_{0.5}$ dispersed in EtOH with PVA dissolved in DMSO. After evaporation of a consequent fraction of EtOH a homogeneous, extrudable paste was
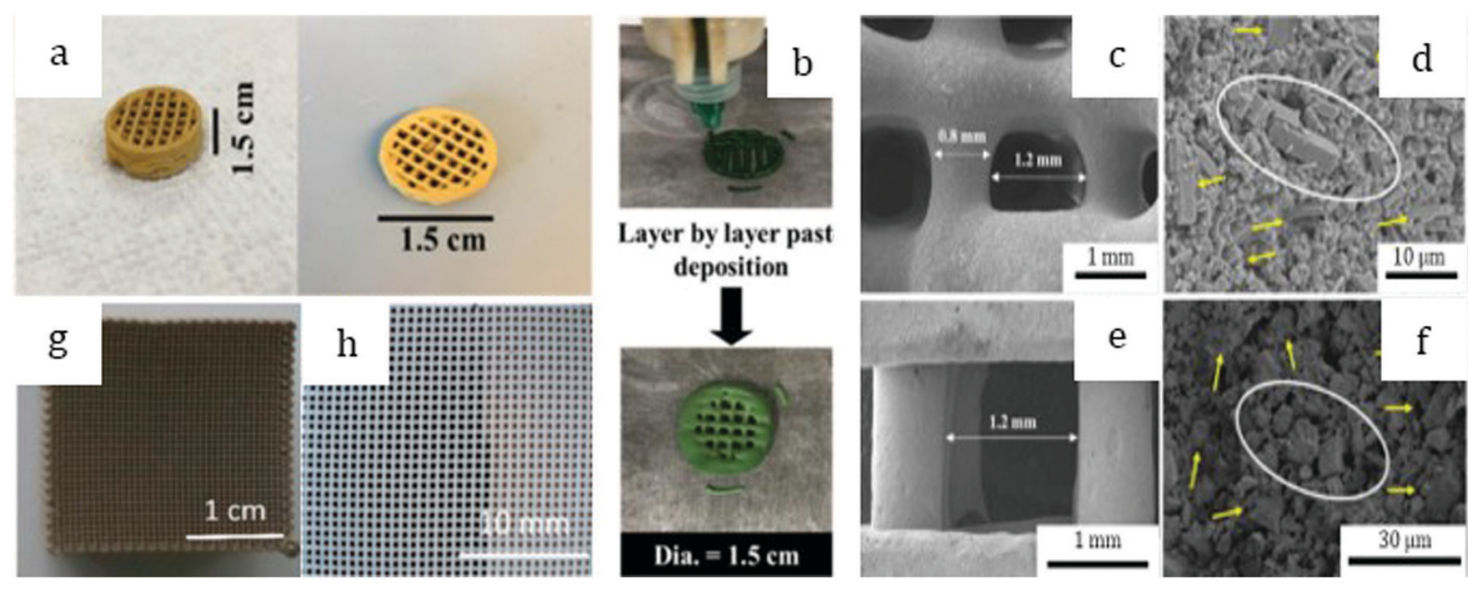

Fig. 11 Photographs of objects 3D-printed via the DIW method: monoliths (a) prepared by Thakkar et al. ${ }^{107}$ Copyright 2017 American Chemical Society. $\mathrm{Ni}(\mathrm{bdc})(\mathrm{ted})_{0.5}$ monolith (b) with its SEM images (c and d) and SEM images of ZIF-7 (e and f) monoliths prepared by Thakkar et al. ${ }^{108}$ Copyright 2018 American Chemical Society. ZIF-8 monolith (g) and its optical image (h) made by Lefevere et al. ${ }^{112}$ Copyright 2019 American Chemical Society. 
obtained. In the case of ZIF-7, the paste was formed by first mixing the pre-synthesized MOF powder and silica with the subsequent addition of a water:methanol mixture. Upon mixing at $60{ }^{\circ} \mathrm{C}$, the composition turned into a smooth, homogeneous paste. Each paste was loaded into a $3 \mathrm{~mL}$ tube equipped with a piston and extruded through a $0.85 \mathrm{~mm}$ nozzle into monoliths in a layer-by-layer manner. Eventually, the thusformed 3D objects were dried at $100{ }^{\circ} \mathrm{C}$ for a few hours (Fig. 11b-f).

The XRD patterns of the monoliths were found to be comparable to those of their powder analogues, suggesting that the crystal structure was retained upon shaping. The intensities however experienced a certain decrease, which was attributed to the presence of PVA. Further analyses revealed pronounced textural properties for $\mathrm{Ni}(\mathrm{bdc})(\mathrm{ted})_{0.5}$ as given by $\mathrm{N}_{2}$ physisorption. Its monolithic form exhibited a $S_{\mathrm{BET}}$ of $1325 \mathrm{~m}^{2} \mathrm{~g}^{-1}$, while its powder form presented a $S_{\text {BET }}$ of $1802 \mathrm{~m}^{2} \mathrm{~g}^{-1}$. The difference was $27 \%$, a value which agrees well with the initial MOF content in the paste (80 wt\%). The corresponding values for ZIF-7 were 16 and $40 \mathrm{~m}^{2} \mathrm{~g}^{-1}$, respectively, for its powder and printed forms. Its porosity is inaccessible to $\mathrm{N}_{2}$ and the slightly higher available surface area was attributed to the silica binder in the printed composition. Interestingly, conventional compression tests revealed an excellent mechanical stability of up to $1.7 \mathrm{MPa}$ for $\mathrm{Ni}(\mathrm{bdc})(\text { ted) })_{0.5}$ due to the high content of binder (20 wt\%), which provided considerably strong bonding of particles. At the same time, ZIF7 monoliths withstood compression up to $0.8 \mathrm{MPa}$, showing that silica might be less appropriate than PVA for strongly bonding MOF particles. When probed for ethane/ethylene adsorption, $\mathrm{Ni}(\mathrm{bdc})(\mathrm{ted})_{0.5}$ monoliths showed total uptakes of 4.1 and $2.9 \mathrm{mmol} \mathrm{g}^{-1}$, respectively. These values were found to be proportional to the MOF content. Notably, ZIF-7 monoliths showed total uptakes of 1.8 and $2.5 \mathrm{mmol} \mathrm{g}^{-1}$, respectively. Both isotherms exhibited an S-shape, revealing the poreopening feature of this MOF upon increasing pressure.

In another study, Lawson et al. ${ }^{109}$ addressed the 3D printing of Cr-based MIL-101 for $\mathrm{CO}_{2}$ capture. The paste formation and printing processes were similar to those reported by Thakkar et $a .^{107}$ Eventually, they succeeded in printing MIL-101 monoliths with bentonite clay (15 wt\%) as the binder and PVA ( $5 \mathrm{wt} \%$ ) as the plasticizer. Such a composition yielded printed objects with a $S_{\mathrm{BET}}$ of $2200 \mathrm{~m}^{2} \mathrm{~g}^{-1}$, which is only $\sim 8 \%$ less than that of the pristine powder $\left(2400 \mathrm{~m}^{2} \mathrm{~g}^{-1}\right)$. The corresponding $\mathrm{CO}_{2}$ uptakes at 1 bar and $25{ }^{\circ} \mathrm{C}$ were 0.75 and $1.0 \mathrm{mmol} \mathrm{g}^{-1}$, respectively. Notably, the decrease in the uptake is consistent with the MOF loading ( $80 \mathrm{wt} \%$ ). Besides, the authors functionalized the monoliths with amino groups via pre- and postimpregnation using polyethylenimine (PEI) and tetraethylenepentamine (TEPA) to enhance the $\mathrm{CO}_{2}$ capture. The presence of amino moieties was confirmed by FTIR analysis through a broad contribution in the range of $3600-2750 \mathrm{~cm}^{-1}$, while the intrinsic MOF peaks were preserved. This suggests the structural integrity of the MOF upon printing. Interestingly, TEPAMIL-101 and PEI-MIL-101 composite monoliths showed an improved $\mathrm{CO}_{2}$ uptake of, respectively, 2.6 and $1.7 \mathrm{mmol} \mathrm{g}^{-1}$.
Table $12 \mathrm{CO}_{2}$ adsorption capacities of the MOFs prepared by Lawson et al. ${ }^{109}$ under $3000 \mathrm{ppm} \mathrm{CO}_{2}$ in $\mathrm{N}_{2}$ at $25^{\circ} \mathrm{C}$

\begin{tabular}{llll}
\hline & \multicolumn{2}{l}{$\mathrm{CO}_{2}$ adsorption capacity, mmol g } \\
\cline { 2 - 3 } MOF & Powder & Printed monoliths & Ref. \\
\hline UTSA-16 & 1.00 & 0.50 & 107 \\
MOF-74 & 1.25 & 1.00 & 107 \\
Zeolite 13X & 1.40 & 1.20 & 96 \\
Zeolite 5A & 1.45 & 1.25 & 96 \\
TEPA-MIL-101 & 1.63 & 1.60 & 109
\end{tabular}

Moreover, the impregnated objects demonstrated higher values of $\mathrm{CO}_{2}$ capture under $3000 \mathrm{ppm}$ of $\mathrm{CO}_{2} / \mathrm{N}_{2}$ than commercial benchmark adsorbents (Table 12), making them promising materials for the $\mathrm{CO}_{2}$ capture.

Lawson et al. ${ }^{110}$ also investigated the use of Torlon ${ }^{\circledR}$, which is a family of polyamide-imides with good mechanical, chemical and thermal stability, as a binder. The mixture of Torlon ${ }^{\circledR}$, MOF powder (HKUST-1 or MOF-74(Ni)), $N$-methyl-2pyrrolidone (NMP), water and DMF (for HKUST-1) was rolled for at least 24 hours prior to use. Woodpile structures with channels of $1 \mathrm{~mm}$ were printed, but the resulting printing quality was fairly poor. Moreover, the microporosity of both printed MOF structures, HKUST-1 and MOF-74(Ni), was drastically reduced. For instance, the HKUST-1 solid printed with $37.5 \mathrm{wt} \%$ Torlon ${ }^{\circledR}$ displayed a $S_{\text {BET }}$ of $290 \mathrm{~m}^{2} \mathrm{~g}^{-1}$, while the MOF-74(Ni) structure collapsed following hydrolysis as observed by PXRD. Notably, the MOF/Torlon ${ }^{\circledR}$ composites presented a mechanical resistance of over $637 \mathrm{MPa}$, three magnitudes higher than that of the solids obtained with bentonite (0.48-0.56 MPa). Their $\mathrm{CO}_{2}$ adsorption capacity at $25{ }^{\circ} \mathrm{C}$ was examined, but the resulting capacities were lower than those previously mentioned $\left(0.6 \mathrm{mmol} \mathrm{g}^{-1}\right.$ for HKUST-1/ Torlon ${ }^{\circledR}$ and $0.3 \mathrm{mmol} \mathrm{g}^{-1}$ for MOF-74(Ni)/Torlon $\left.{ }^{\circledR}\right)$.

Finally, Lawson et al. ${ }^{111}$ studied the post-printing crystallization of HKUST-1 starting from a gel containing all precursors. In this case, a mixture of bentonite (21 wt\%), methylcellulose (2 wt\%) and PVA (6 wt\%) was used to obtain satisfactory rheological properties. The as-printed grids presented a fair replication of the initial model, and they were further placed in a convection oven at $120{ }^{\circ} \mathrm{C}$ for 20 hours to induce crystallization of the MOF. The resulting material presented a $S_{\text {BET }}$ of $500 \mathrm{~m}^{2} \mathrm{~g}^{-1}$, slightly higher than that of a comparative solid directly 3D-printed starting from the HKUST1 powder $\left(470 \mathrm{~m}^{2} \mathrm{~g}^{-1}\right)$. While the solids were extensively washed with acetone, residual DMF was observed by FTIR spectroscopy as characterized by a band at $2100 \mathrm{~cm}^{-1}$. Finally, the $\mathrm{CO}_{2}$ capacities of both solids at $25{ }^{\circ} \mathrm{C}$ were compared. While the solid prepared from the HKUST-1 powder presented a $\mathrm{CO}_{2}$ capacity $50 \%$ higher $\left(2.1 \mathrm{mmol} \mathrm{g}^{-1}\right.$ against $\left.1.4 \mathrm{mmol} \mathrm{g}^{-1}\right)$, which is not in line with their respective $S_{\mathrm{BET}}$, the solid obtained by growing HKUST-1 crystals on the as-printed solid displayed enhanced mass transfer kinetics (diffusivity $\times 10^{8}$ $\left(\mathrm{cm}^{2} \mathrm{~s}^{-1}\right): 8.75$ against 5.25). This was attributed to the presence of a larger extent of mesopores $\left(V_{\text {meso }}\left(\mathrm{cm}^{3} \mathrm{~g}^{-1}\right.\right.$ STP) $=0.16$ against 0.09 ). 
Further reports on shaping ZIF-8 via DIW include the work by Lefevere et al., ${ }^{112}$ who managed to formulate the MOF with a blend of inorganic and organic binders. The former was added to improve the mechanical stability of the shaped objects, and the latter to enhance the rheological properties of the paste. Typically, the parent ZIF-8 powder (66.7 wt\%) was mixed with bentonite $(16.7 \mathrm{wt} \%)$ and methylcellulose (16.7 wt\%) with a subsequent addition of water and mixing to form a homogeneous printable paste. Once homogenized, it was further loaded into a $50 \mathrm{~mL}$ syringe and extruded through 250 and $600 \mu \mathrm{m}$ diameter nozzles in a layer-by-layer fashion at room temperature (Fig. $11 \mathrm{~g}$ and $\mathrm{h}$ ).

Upon printing, the objects were thermally treated in an inert atmosphere to remove the organic binder. It was found that elimination of methyl cellulose without structural degradation of ZIF-8 took place at $450{ }^{\circ} \mathrm{C}$. Upon such thermal treatment, the ZIF- 8 and bentonite contents in the printed objects were 80 and $20 \mathrm{wt} \%$, respectively. The crystal structure remained intact upon thermal activation as confirmed by XRD diffractograms. Besides, IR analysis demonstrated the preservation of the bands characteristic of the original MOF powder, with additional bands at $1100 \mathrm{~cm}^{-1}$ corresponding to $\mathrm{Si}-\mathrm{O}$ stretching, implying the presence of bentonite. Ar physisorption measurements revealed a $24 \%$ decrease of $S_{\mathrm{BET}}$, from $1415 \mathrm{~m}^{2} \mathrm{~g}^{-1}$ (ZIF-8 powder) to $1070 \mathrm{~m}^{2} \mathrm{~g}^{-1}$ (ZIF-8 monolith). This decrease is consistent with the binder content and suggests only a minor pore blocking. Further adsorption studies of a gas mixture (ethanol, $n$-butanol, isopropanol and water) on ZIF-8 monoliths demonstrated the preferential adsorption of $n$-butanol at $40{ }^{\circ} \mathrm{C}$ as it eluted the last, with a breakthrough time of $43 \mathrm{~h}$. Further studies on $n$-butanol adsorption from an acetone-butanolethanol (ABE) gas mixture over 3D printed ZIF-8 were done by Claessens et al. $^{113}$ They showed a higher affinity of $n$-butanol towards ZIF-8 monoliths and therefore their eligibility for the separation of $n$-butanol from the ABE gas mixture.

$3 \mathrm{D}$ printing also enabled the preparation of porous conducting matrices as cathode supports based on MOFs for $\mathrm{Li}^{-\mathrm{O}_{2}}$ batteries in a study by Lyu et al. ${ }^{114}$ For this purpose, they chose Co-based ZIF-67 which was mixed with Pluronic F127 in water to form a printable ink. The latter was used to generate a hierarchical porosity within the shaped objects. The paste was further transferred into a $20 \mathrm{~mL}$ syringe equipped with nozzles of up to $400 \mu \mathrm{m}$ and printed in a layer-by-layer manner. Upon printing, the composites were dried and decomposed at $800{ }^{\circ} \mathrm{C}$ under $\mathrm{N}_{2}$ to form Co nanoparticles entrapped in $\mathrm{N}$-doped mesoporous carbon flakes. These materials exhibited a BET surface area of $640 \mathrm{~m}^{2} \mathrm{~g}^{-1}$. The thus-formed Co-based electrocatalysts increased the cell's specific energy and therefore the energy density value up to $798 \mathrm{~W} \mathrm{~h} \mathrm{~kg}_{\text {cell }}{ }^{-1}$, which is higher than those of the currently well-established Li-ion batteries. This suggests the integration of MOF-derived carbon-based nanomaterials as promising composites for advanced battery systems.

A detailed study on the rheology of printable inks was done by Dhainaut et al. $^{115}$ on a series of MOFs. Namely, Cu-based CPL-1 and HKUST-1, Zr-based UiO-66- $\mathrm{NH}_{2}$, and Zn-based ZIF-8 inks were formulated. A typical paste was prepared by mixing a predefined amount of the parent MOF, polyvinyl alcohol (PVA) as the plasticizer, and a gel of 2-hydroxyethyl cellulose (HEC) as the binder. The paste was further extruded through a $0.8 \mathrm{~mm}$ diameter needle in a layer-by-layer manner on a surface preheated at $60{ }^{\circ} \mathrm{C}$. Eventually, the thus-printed objects were exposed to vacuum drying at $90{ }^{\circ} \mathrm{C}$ (Fig. 12a and b). All crystal structures were retained upon printing, as confirmed by matching XRD patterns. A decrease in the intensities of the reflections was attributed to the presence of organic additives within the $3 \mathrm{D}$ objects. Besides, the gas $\left(\mathrm{N}_{2}\right.$ and $\left.\mathrm{CO}_{2}\right)$ sorption capacities of these materials experienced a certain drop upon printing; however, the values mostly remained in the range of the organics' percentages (Table 11), as estimated by TGA measurements. As evidenced from Table 11, HKUST-1 experienced a higher $(24 \%)$ loss of $S_{\mathrm{BET}}$ as compared to the estimated content of organic additives. This can be explained by the partial degradation of the framework upon dispersion in a water/ ethanol mixture during the paste formation step. Nevertheless, methane adsorption experiments revealed decent uptakes of 81,55 and $38 \mathrm{~g} \mathrm{~kg}^{-1}$, respectively, for printed HKUST-1, ZIF-8 and UiO-66- $\mathrm{NH}_{2}$ monoliths at $25{ }^{\circ} \mathrm{C}$ and 40 bar. The values are
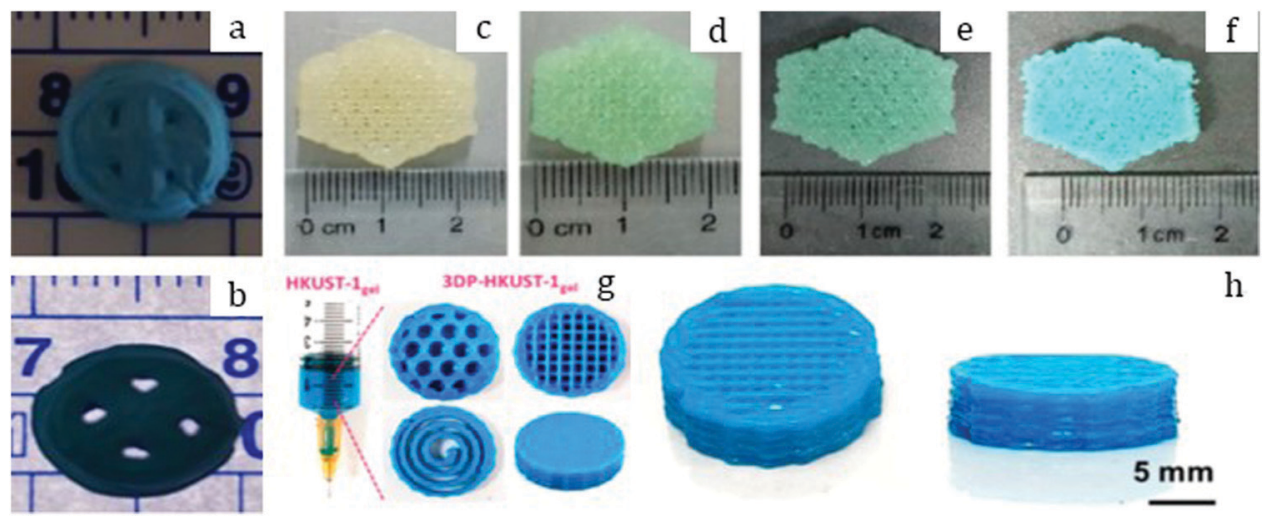

$\mathrm{h}$

Fig. 12 Photographs of objects 3D-printed via the DIW method: CPL-1 before (a) and after drying (b) prepared by Dhainaut et al. ${ }^{115}$ Copyright 2020 American Chemical Society. Printed objects with HKUST-1 loadings of $0 \%$ (c), $2.5 \%$ (d), $3.7 \%$ (e) and $13 \%$ (f) prepared by Pei et al. ${ }^{116}$ Copyright 2020 Elsevier. HKUST-1 gel in a syringe $(\mathrm{g})$ and monoliths ( $\mathrm{g}$ and $\mathrm{h}$ ) prepared by Lim et al. ${ }^{117}$ Copyright 2019 American Chemical Society. 
close to the ones reported for their powder analogues. Interestingly, adsorption of ethane/ethylene gas over printed CPL-1 monoliths revealed no ethane uptake above 8 bar, while adsorption of ethylene increased linearly up to 16 bar, thus making this MOF a highly-preferential ethylene adsorbent.

Direct ink writing of HKUST-1 was also reported by Pei et $a l .{ }^{116}$ The paste was formed by mixing a pre-synthesized HKUST-1 powder with sodium alginate and gelatin in water until a homogeneous ink was formed. The latter was then printed through a $0.21 \mathrm{~mm}$ nozzle in a layer-by-layer manner at room temperature. The thus-formed objects were then immersed in a $\mathrm{CaCl}_{2}$ solution to initiate cross-linking and eventually exposed to drying at $80{ }^{\circ} \mathrm{C}$ to obtain the final objects (Fig. 12c-f). As confirmed by XRD, the printed HKUST-1 objects exhibited reflections characteristic of the original powder, suggesting that the crystal structure remained intact upon printing. Besides, the $S_{\text {BET }}$ was $83 \mathrm{~m}^{2} \mathrm{~g}^{-1}$ at $\sim 13 \mathrm{wt} \%$ HKUST-1 loading, while its powder counterpart reached $1563 \mathrm{~m}^{2} \mathrm{~g}^{-1}$, suggesting a drastic 95\% loss and therefore pore blocking caused by the alginate species and gelatin. Nevertheless, it was shown that the printed objects retained the adsorptive properties of the parent MOF towards the most common dyes and exhibited recyclability properties. Thus, the removal efficiency of the 3D printed objects towards methylene blue increased from 43.6 to 79.5 , 90 and $96 \%$ as the HKUST-1 loading increased from 0 to $2.5,3.7$ and $13 \%$, respectively. The results were consistent upon 10 consecutive adsorptionregeneration cycles.

The binderless shaping of HKUST-1 via DIW was reported by Lim et al. ${ }^{117}$ They demonstrated that a colloidal gel containing only the parent MOF dispersed in ethanol can serve as an ink for printing at room temperature. This paste was extruded through a syringe with a $300 \mu \mathrm{m}$ diameter tip to yield circular objects with a diameter of $15 \mathrm{~mm}$ and a thickness of $2 \mathrm{~mm}$. Eventually, the thus-shaped objects were dried in a desiccator (Fig. $12 \mathrm{~g}$ and $\mathrm{h}$ ).

According to XRD results, the printed objects retained the original crystal structure of HKUST-1 upon formulation. A certain peak broadening was observed for all materials, suggesting small MOF crystals. Indeed, as confirmed by SEM, the shaped objects were composed of HKUST-1 crystals with sizes in the 20-50 nm range. However, a significant decrease of the $S_{\text {BET }}$ was measured, from $1850 \mathrm{~m}^{2} \mathrm{~g}^{-1}$ for the parent powder to $1134 \mathrm{~m}^{2} \mathrm{~g}^{-1}$ for the 3D-printed solids. As no binder was present, this decrease might be ascribed to the partial collapse of the HKUST-1 framework.

Lately, Grande et al. applied 3D-printed UTSA-16 solids for selective $\mathrm{CO}_{2}$ capture. ${ }^{118}$ For the binding system, they used a mixture of boehmite and hydroxypropyl cellulose, representing $36 \mathrm{wt} \%$ of the final dry solid content. Interestingly, the presence of boehmite might provide some Lewis acidity, which was not probed. The as-obtained solids with a diameter of $28 \mathrm{~mm}$ presented a woodpile structure with only little sagging in the middle. After activation, the $S_{\mathrm{BET}}$ reached $540 \mathrm{~m}^{2} \mathrm{~g}^{-1}$, which is higher than expected as UTSA-16 powder typically presents a $S_{\text {BET }}$ of $630 \mathrm{~m}^{2} \mathrm{~g}^{-1}$. While the solids retained selectively $\mathrm{CO}_{2}$ over $\mathrm{N}_{2}$, the additional presence of water molecules desorbed the $\mathrm{CO}_{2}$ molecules.

A one-pot approach based on the in situ growth of ZIF-8 and MIL-100 MOFs onto anionic cellulose nanofibers (TOCNF) was first described by Sultan et al. ${ }^{119}$ The latter acts as an anchor for stabilizing ionic $\mathrm{Zn}$ and Fe species, prior to their precipitation in the presence of triethylamine. The water-based ink was formulated by mixing the metal precursor solution, TOCNF, TEA, and the linker solution. Prior to printing, sodium alginate was added as a plasticizer to improve the ink printability.
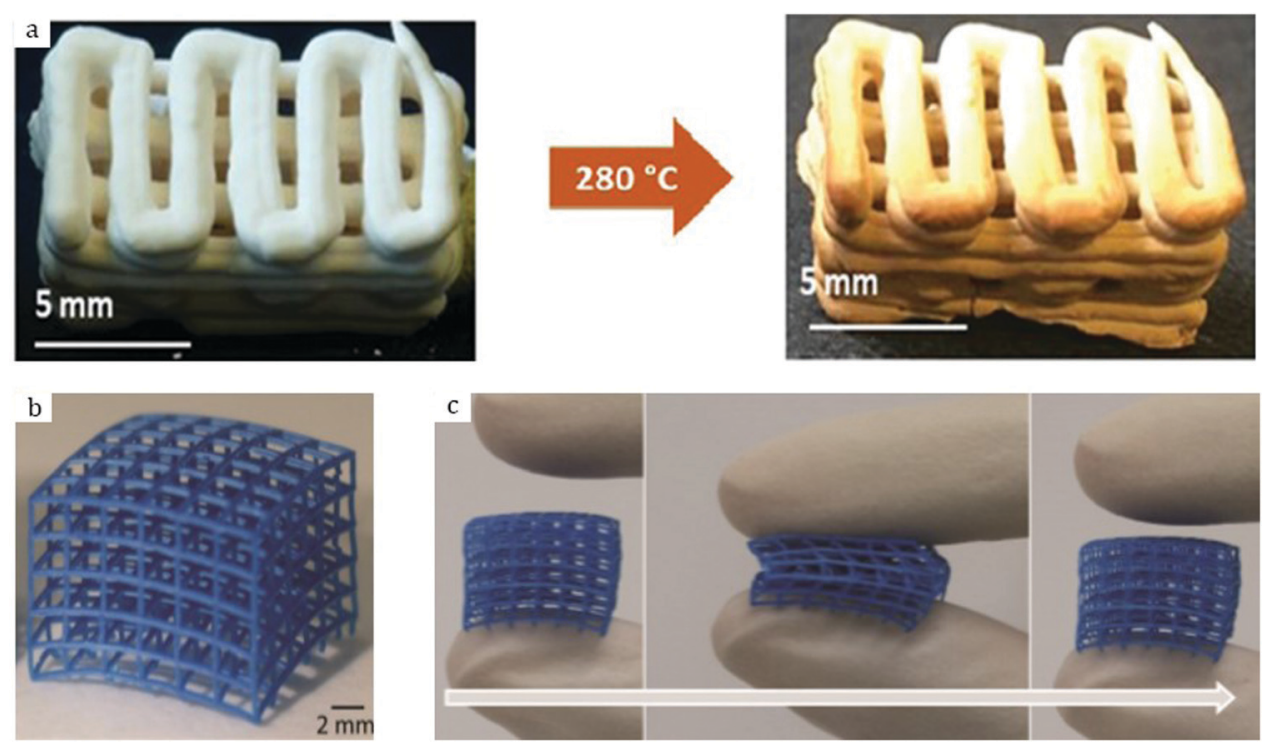

Fig. 13 Photographs of objects 3D-printed via the DLP method: UiO-66 composite (a) before and after drying prepared by Young et al. ${ }^{120}$ (the color change from white to light-brown was attributed to the polymer and photoinitiator degradations). Copyright 2019 Royal Society of Chemistry. HKUST-1/ polymer composite (b) and demonstration of its flexibility (c) prepared by Halevi et al. ${ }^{121}$ Copyright 2017 Wiley. 
Monoliths of $8 \mathrm{~cm}^{3}$, with a pore size of $1 \mathrm{~mm}$ and a wall thickness of $0.5 \mathrm{~mm}$, were obtained following ink deposition using a $410 \mu \mathrm{m}$ diameter nozzle and a printing speed of 10$80 \mathrm{~mm} \mathrm{~s}^{-1}$. This protocol was finalized by subsequent soaking of the printed object in a calcium chloride solution, acting as a cross-linking agent. Furthermore, it was shown that drugs can be encapsulated into the printed objects with a subsequent $\mathrm{pH}$ dependent release. Thus, curcumin, an anticancer drug, was encapsulated in both TOCNF/ZIF-8 and TOCNF/MIL-101, while methylene blue, a hemoglobin-regulating drug, was incorporated into TOCNF/ZIF-8. Hence, 3D printing was demonstrated to be suitable for preparing MOF-based drug carriers. Both active compounds were shown to be released under acidic conditions at $\mathrm{pH} \sim 5.5$.

\section{UV irradiation-assisted 3D printing, digital light processing (DLP)}

Young et $a .^{120}$ used a DLP approach to print MOF-based objects. In their work, the ink was formulated by mixing the parent MOF with ethanol and adding an acrylate-based polymer binder (trimethylolpropane propoxylate triacrylate (TMPPTA) and EBECRYL ${ }^{\mathbb{R}}$ 8413) as well as an initiator blend (phenylbis(2,4,6-trimethylbenzoyl)phosphine oxide in 2hydroxy-2-methylpropiophenone). The thus-formed paste with a total MOF content of $52 \mathrm{wt} \%$ was further loaded into a $25 \mathrm{~mL}$ syringe and extruded through a 16-gauge $(\sim 1.3 \mathrm{~mm})$ needle at room temperature. The objects were irradiated with $365 \mathrm{~nm} \mathrm{UV-}$ light during printing prior to heating at $280{ }^{\circ} \mathrm{C}$ for $30 \mathrm{~min}$ (Fig. 13a). This was followed by washing with dichloromethane and drying at $50{ }^{\circ} \mathrm{C}$ under vacuum. The printed objects retained the crystal structure of the original UiO-66 powder as was evident from the matching XRD patterns and FTIR bands. Besides, a band corresponding to vinyl groups (at around $2900 \mathrm{~cm}^{-1}$ ) vanished upon printing, indicating the successful polymerization of the binder following UV-light irradiation. Furthermore, the binder partially liberated the porosity when removed by heating, yielding a specific surface area of $633 \mathrm{~m}^{2} \mathrm{~g}^{-1}$, while that of its powder analogue was $1590 \mathrm{~m}^{2} \mathrm{~g}^{-1}$.

A similar approach was developed by Halevi et al., ${ }^{121}$ starting from Cu-based HKUST-1 powder to obtain MOF-embedded flexible polymeric structures. For this purpose, they used a blend of 2-phenoxyethyl acrylate (PEA) and polyethylene glycol diacrylate (PGD) as the polymeric matrix, while Irgacure-819 and Irgacure-184 were applied as photoinitiators. The ink containing all components and a MOF content of $10 \mathrm{wt} \%$ was loaded into a DLP 3D printer to yield flexible HKUST-1/polymer 3D composites (Fig. 13b and c). The use of a hydrophobic polymer matrix enhanced the hydrolytic stability of the watersensitive HKUST-1 and enabled its use for the adsorption of methylene blue (MB) in an aqueous medium. Thus, the HKUST1/polymer composite retained around $6.5 \mathrm{mg} \mathrm{g}^{-1}$ of $\mathrm{MB}$, which is comparable to the $7 \mathrm{mg} \mathrm{g}^{-1}$ capacity of the pristine MOF powder. However, for a longer adsorption duration (around $9 \mathrm{~h}$ ) the latter released MB back into the aqueous solution, indicating its structural collapse due to hydrolysis. Instead, the $3 \mathrm{D}$ printed composite retained the adsorbed MB after $9 \mathrm{~h}$, owing to its enhanced stability in water.

Liu et al. developed a DLP approach based on the use of a DIW 3D printer. ${ }^{122}$ For that, they formulated an ink in which, in addition to gelling agents (hydroxyethyl cellulose and sodium alginate), $25 \mathrm{wt} \%$ acrylamide and $0.4 \mathrm{wt} \%$ of a photoinitiator were also added. The resulting solids were photocured for 15 minutes, prior to immersion in a suspension containing HKUST-1 precursors. After 24 hours, HKUST-1 crystals were formed onto and within the photocured solids as observed by SEM. However, the main reflection of HKUST-1 was barely visible by $\mathrm{XRD}$, indicating that at least some of the $\mathrm{Cu}^{2+}$ remained amorphous. The resulting MOF composites
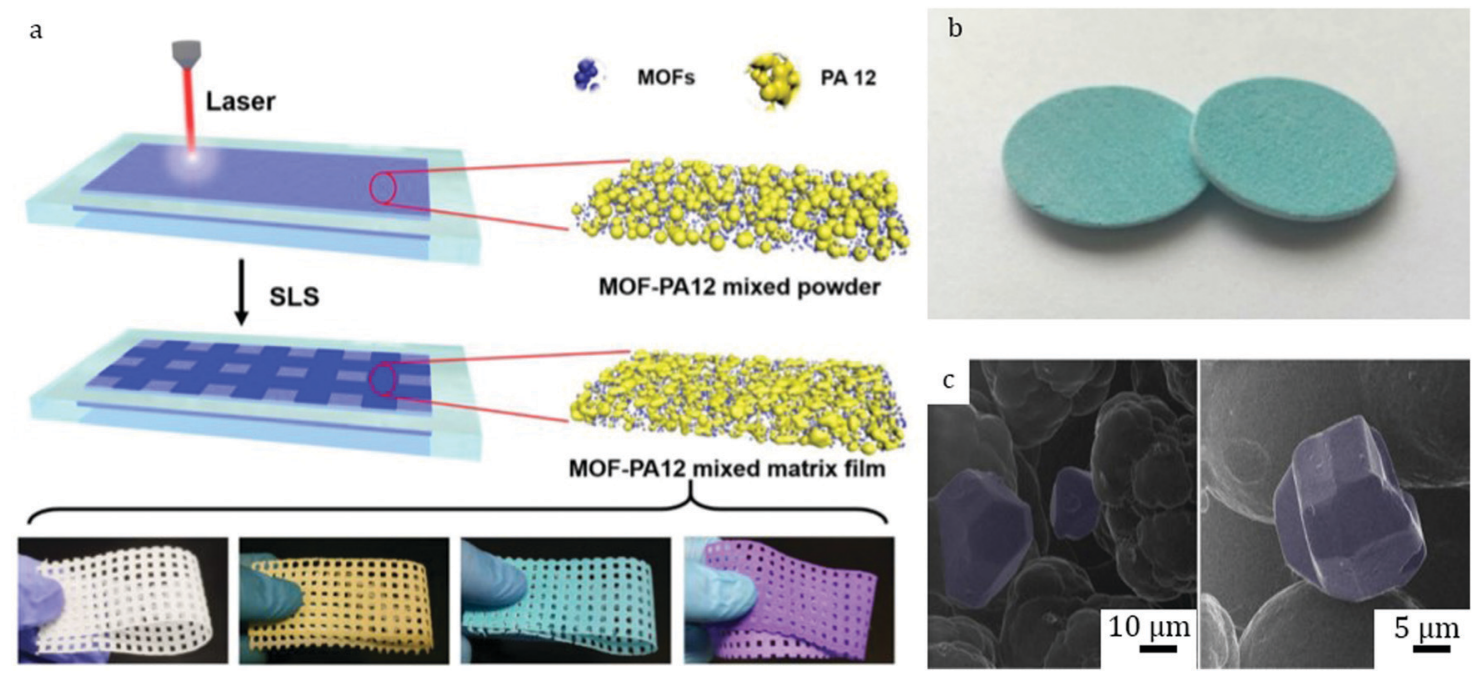

Fig. 14 Schematic representation of the SLS 3D printing process (a, top) and photograph of printed objects: MOF/polymer matrices (a, bottom) from left to right: PA12 film, $\mathrm{NH}_{2}$-MIL-101/PA12, HKUST-1/PA12, ZIF-67/PA12 prepared by Li et al. ${ }^{123}$ Copyright 2019 American Chemical Society. MOF/PA12 composites with $20 \mathrm{~mm}$ diameter and $1.5 \mathrm{~mm}$ thickness (b) and HIM images of their surfaces with MOF particles highlighted in purple (c) prepared by Lahtinen et al. ${ }^{124}$ Copyright 2019 Wiley. 
presented a large shape deformation (453\%) and a high tensile strength $(278 \mathrm{kPa})$. When applied to MB adsorption $(30 \mu \mathrm{m}$ in water), full adsorption was observed after 5 hours. Unfortunately, the recyclability was not reported.

\section{Laser-assisted 3D printing, selective laser sintering (SLS)}

The SLS technology was successfully employed by Li et al. ${ }^{123}$ to produce a series of MOF-polymer mixed matrix films (MMFs) starting from polyamide 12 (PA12) and five different MOFs: ZIF67, $\mathrm{NH}_{2}$-MIL-101, MOF-801, HKUST-1 and ZIF-8. Each of the MOF powders was thoroughly mixed with the matrix before deposition as a thin layer on a quartz support. Then, a $\mathrm{CO}_{2}$ laser beam $(\lambda \sim 10.6 \mu \mathrm{m})$ was applied at a scanning speed of $1.78 \mathrm{~m} \mathrm{~s}^{-1}$ and a laser power of $9.6 \mathrm{~W}$ (16\% of maximal power, to avoid MOF decomposition). Eventually, MMFs with differently-sized grid patterns and MOF contents up to $40 \mathrm{wt} \%$ were formed (Fig. 14a). As confirmed by the XRD analysis, the thus-formed films retained the initial MOF crystal structure while also exhibiting broad reflections attributed to PA12. Besides, the MMF containing $40 \mathrm{wt} \%$ ZIF-8 exhibited a surface area of $332 \mathrm{~m}^{2} \mathrm{~g}^{-1}$, against $1359 \mathrm{~m}^{2} \mathrm{~g}^{-1}$ for pure ZIF-8 powder. Hence, while partial pore blockage was caused by the PA12 matrix, the MOF particles were not encapsulated into the polymer matrix and remained accessible for adsorption of guest molecules. Thus, the MMF containing $\mathrm{NH}_{2}-\mathrm{MIL}-101$ was demonstrated to be as efficient as its powder counterpart for methylene blue adsorption ( $81 \%$ removal efficiency), and it retained its performance after 5 adsorption-desorption cycles.

The same SLS method was applied by Lahtinen $e t$ al. ${ }^{124}$ to produce HKUST-1/PA12 porous filters with a MOF loading of $10 \mathrm{wt} \%$. A $14 \mathrm{~W} \mathrm{CO}_{2}$ laser operating at $40 \%$ laser power and $2.4 \mathrm{~m} \mathrm{~s}^{-1}$ laser speed was employed to obtain the composites (Fig. 14b and c). The temperature of printing was set to $170{ }^{\circ} \mathrm{C}$, which was low enough to retain the crystal structure of HKUST1 within the polymer matrix, as confirmed by XRD. Besides, HKUST-1/PA12 pellet-like composites exhibited a moderate $S_{\mathrm{BET}}$ of $40 \mathrm{~m}^{2} \mathrm{~g}^{-1}$, which was attributed to the low MOF content. Nevertheless, the printed filters presented an adsorption capacity for $\mathrm{CO}_{2}$ in line with the MOF content, reaching a $0.6 \mathrm{wt} \%$ increase in mass upon adsorption, while the pristine powder exhibited a $6.0 \mathrm{wt} \%$ increase. This suggests that the SLS technique brings no significant alteration of the structural and chemical properties of pristine MOF powders.

To sum up, 3D printing is a relatively new shaping technique which is based on extrusion through a thin tip in a controlled manner. This grants 3D printing some essential advantages over classical extrusion such as a wide variety of possible shapes to form. However, proper control of the rheological properties is crucial in the case of $3 \mathrm{D}$ printing as the paste should retain its form once deposited on a substrate. Besides, MOFs can be introduced into a polymer matrix depending on the nature of the latter in different ways (by SLS, DLP or MOF growth on the printed polymer matrix). At the same time, direct writing of MOF-based inks has also been successfully developed. Comparative data are given in Table 13.
Table 13 Comparison of DIW 3D printing and manual extrusion

\begin{tabular}{llllll}
\hline & \multicolumn{3}{c}{ Binder, } & & \multicolumn{2}{c}{ Loss of } \\
Technique & MOF & wt\% & Plasticizer & SSA, \% & Ref. \\
\hline 3D printing (DIW) & HKUST-1 & HEC, 19.5 & PVA & -25 & 115 \\
3D printing (DIW) & ZIF-8 & Bentonite, 16.7 & MC & -24 & 112 \\
Manual extrusion & HKUST-1 & PVA, 2.9 & $\mathrm{H}_{2} \mathrm{O} / \mathrm{PrOH}$ & -5 & 84 \\
Manual extrusion & ZIF-8 & PVA, 2.9 & $\mathrm{H}_{2} \mathrm{O} / \mathrm{PrOH}$ & -2 & 84
\end{tabular}

As is evident from Table 13, both extrusion and DIW can yield shaped objects while preserving the textural properties of pristine MOFs. The loss in surface area in the case of the 3D printed objects is somewhat higher than the binder content which is due to the presence of the plasticizer. The final printed objects have a developed network of millimeter-sized channels thanks to the layer-by-layer deposition upon printing. This can significantly improve the diffusion of gas or liquid within the shaped objects.

\section{Spray-drying}

The spray-drying technique has been known for over a century. As the name suggests, it is used to dry powder-like materials in a continuous fashion. Compared with ovens and conveyor belts, spray-dryers allow treating several tons per hour of wet product. Over the past few decades, the application potential of spray-drying has considerably expanded and now includes the food industry, ${ }^{125}$ the pharmaceutical industry, ${ }^{126}$ and many others. ${ }^{127}$

The basic principle behind the spray-drying procedure lies in the atomization of a slurry into micron-sized droplets within a heated chamber. During this process, the droplets containing the dispersed powder are quickly evaporated, leaving the dried powder to be further captured and collected. The spray-drying process strongly relies on the following three parameters: the inlet temperature $\left(T_{\text {in }}\right)$ up to which the circulating gas is heated, flow rate at which the gas is introduced in the chamber, and feed rate at which the precursor solution or slurry is injected into the atomizer (Fig. 15a). The variation of these parameters depends on the final purpose of spray-drying.

Interestingly, upon drying, the individual particles dispersed in the slurry feed adopt the shape of the atomized droplets and thus form larger, micron-sized spherical agglomerates. Therefore, the entire spray-drying process can also be viewed as a technique for shaping particles into microbeads, particularly adapted for fluidized-bed reactors. ${ }^{59}$

Moreover, spray-drying allows the direct synthesis of various materials. ${ }^{128}$ In 2002, du Fresne von Hohenesche et al. ${ }^{129}$ successfully prepared MCM-41 spherical microbeads with a defined arrangement of macro- and mesopores with the help of a spray-dryer. Since then, the same approach has been used for preparation of other types of porous materials, ${ }^{130}$ allowing spray-drying to be considered as a tool for simultaneous synthesis and shaping.

A few years ago, the group of D. Maspoch ${ }^{131}$ developed a strategy for a fast, facile and scalable synthesis of MOFs using a 
a

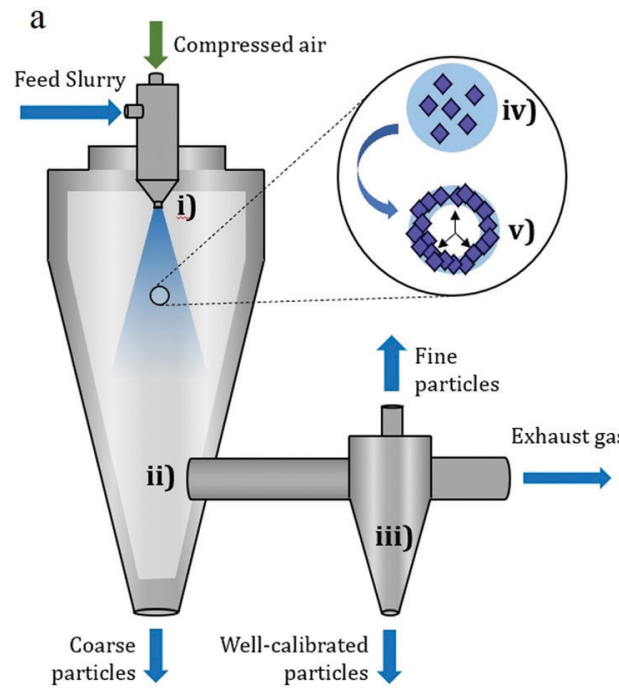

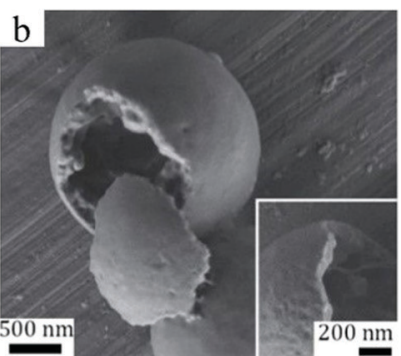
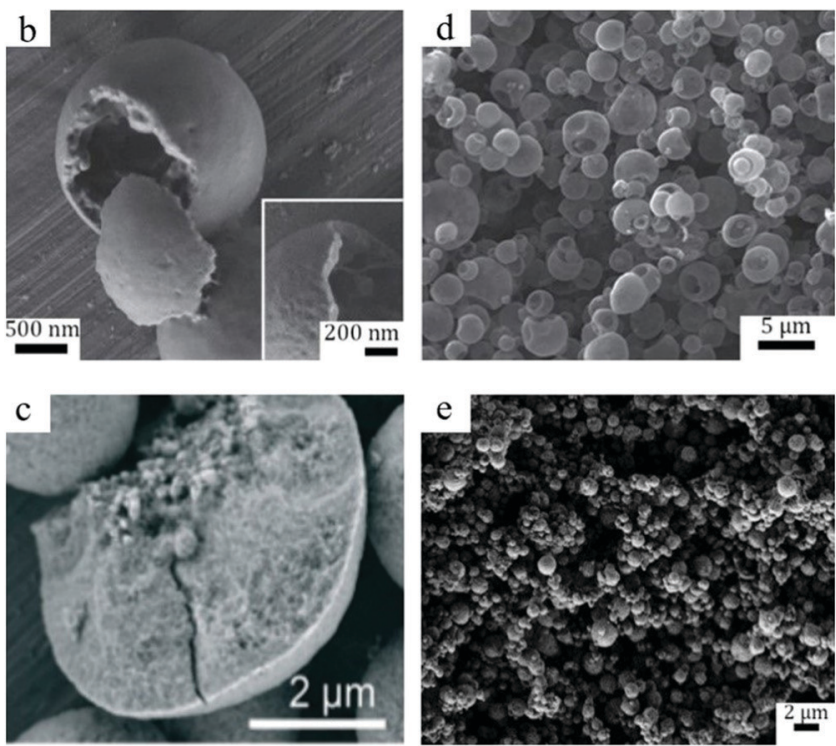

Fig. 15 Schematic representation (a) of the spray-drying process: (i) atomizer; (ii) drying chamber; (iii) cyclone separator for gas/powder separation; (iv) sprayed droplet with a MOF precursor; and (v) dried droplet with MOF crystals at the edge. SEM image of a HKUST-1 hollow bead (b) prepared by CarneSanchez et al. ${ }^{131}$ Copyright 2013 Springer Nature. SEM image of a UiO-66 dense bead (c), prepared by Garzon-Tovar et al. ${ }^{138}$ Copyright 2016 Royal Society of Chemistry. HKUST-1 hollow beads produced with a B-290 Mini Spray Dryer (d) and a Nano Spray Dryer B-90 (e), prepared by Carne-Sanchez et al. ${ }^{131}$ Copyright 2013 Springer Nature.

spray-dryer. In a typical synthesis, the solution (or suspension) containing the MOF precursors is atomized into a chamber. There, upon constant contact with a hot gas, the precursors rapidly react, thus forming MOF nanoparticles inside the droplets. At the same time, they are assembled into dense or hollow spherical structures (Fig. 15b and c). Eventually, the droplets are completely evaporated and freshly formed MOF microbeads are collected. This approach has been successfully applied to a wide variety of MOFs, including some of the moststudied HKUST-1, UiO-66, ZIF-8, and MOF-5, among others. Generally, the final size of the microbeads varies from a few hundreds of nanometers to a few microns.

The final shape of the solid particles might alter the MOF's physical and chemical properties. Some of them are summarized in Table 14 with up-to-date references on MOFs produced by spray-drying.

\section{One step synthesis, hollow structures}

As evidenced from Table 14, the formation of either hollow or dense microbeads upon spray-drying is primarily dictated by the nature of the MOF itself. Thus, HKUST- 1 and ZIF- 8 tend to arrange into spheres with a void core, while the whole family of UiO and its derivatives build up into compact, dense microbeads. This trend was first described by Carnez-Sanchez et al. ${ }^{131}$ in their pioneering work on using spray-drying for the synthesis of MOFs. They were the first to show that individual droplets sprayed in the chamber could be viewed as microreactors to crystallize MOFs and form spherical hollow superstructures which resulted from the assembly of individual nanocrystallites. Indeed, this approach was successfully applied to a large family of MOFs with a particular stress on the Cu-based
HKUST-1. For its preparation, the feed solution was spray-dried at an inlet temperature of $180{ }^{\circ} \mathrm{C}$, a feed rate of $4.5 \mathrm{~mL} \mathrm{~min}^{-1}$ and a flow rate of $336 \mathrm{~mL} \mathrm{~min}{ }^{-1}$. This led to the formation of a blue powder composed of spherical agglomerates (superstructures) with an average size of $2.5 \pm 0.4$ microns and exhibiting the HKUST-1 phase as confirmed by XRD (Fig. 15d). Additionally, the thus-shaped HKUST-1 retained its high porosity, showing a $S_{\text {BET }}$ of $1209 \mathrm{~m}^{2} \mathrm{~g}^{-1}$, a value in agreement with HKUST-1 typically prepared under solvothermal conditions. ${ }^{132}$ Besides, the authors performed a study on the impact of spraydrying conditions on the morphology of the final superstructures. Namely, an increase in the flow rate from 336 up to 474 $\mathrm{mL} \mathrm{min}^{-1}$ led to the formation of considerably smaller discrete HKUST-1 nanoparticles forming the superstructures, from 160 \pm 40 down to $107 \pm 30 \mathrm{~nm}$. At the same time, no considerable impact was observed when decreasing the inlet temperature from 180 down to $100{ }^{\circ} \mathrm{C}$, leaving the mean size unchanged. Interestingly, it was demonstrated that MOF superstructures can be miniaturized by decreasing the size of the sprayed droplets. This can be achieved by using another spray-dryer: for instance, the Nano Spray Dryer B-90 (Büchi Labortechnik) enabled the production of hollow MOF microbeads with an average size of $950 \pm 300 \mathrm{~nm}$ (Fig. 15e).

Another interesting finding was the possibility to synthesize MOFs such as Fe-based MIL-88A, which instantly produce an amorphous phase once their precursors are mixed at room temperature. Spray-drying allows overcoming this issue by using a three-fluid nozzle which ensures that the precursors do not mix before the drying chamber or just before they enter the atomizer (T-junction). The authors further applied this approach to a large family of MOFs (see Table 14). Furthermore, 
Table 14 List of the MOFs produced via the spray-drying technique

\begin{tabular}{|c|c|c|c|c|c|c|c|}
\hline MOF & Metal/ligand & Spray dryer & $T_{\mathrm{in}},{ }^{\circ} \mathrm{C}$ & $\begin{array}{l}\text { Feed rate, } \\
\mathrm{mL} \min ^{-1}\end{array}$ & $\begin{array}{l}\text { Flow rate, } \\
\mathrm{mL} \min ^{-1}\end{array}$ & $S_{\mathrm{BET}}, \mathrm{m}^{2} \mathrm{~g}^{-1}$ & Ref. \\
\hline \multicolumn{8}{|c|}{ One step synthesis, hollow structures } \\
\hline HKUST-1 & $\mathrm{Cu} / \mathrm{BTC}$ & B-290 & 180 & 4.5 & 336 & 1209 & 131 \\
\hline HKUST-1 & $\mathrm{Cu} / \mathrm{BTC}$ & B-290 & 180 & 4.5 & 336 & 1430 & 133 \\
\hline HKUST-1@PS & $\mathrm{Cu} / \mathrm{BTC}$ & B-290 & 130 & 4.5 & 336 & 757 & 133 \\
\hline $\mathrm{Cu}-\mathrm{BDC}$ & $\mathrm{Cu} / \mathrm{BDC}$ & B-290 & 180 & 4.5 & 336 & 543 & 131 \\
\hline NOTT-100 & $\mathrm{Cu} / \mathrm{BPTC}$ & B-290 & 180 & 4.5 & 336 & 1140 & 131 \\
\hline $\mathrm{Cu}-\mathrm{PB}$ & $\mathrm{Cu} / \mathrm{K}_{3} \mathrm{Co}(\mathrm{CN})_{6}$ & B-290 & 180 & 4.5 & 474 & 617 & 131 \\
\hline MOF-5 & $\mathrm{Zn} / \mathrm{BDC}$ & B-290 & 180 & 4.5 & 336 & 1215 & 131 \\
\hline MOF-14 & $\mathrm{Cu} / \mathrm{BTB}$ & B-290 & 180 & 4.5 & 336 & - & 131 \\
\hline Zn-MOF-74 & Zn/DHBDC & B-290 & 180 & 4.5 & 336 & - & 131 \\
\hline Mg-MOF-74 & $\mathrm{Mg} / \mathrm{DHBDC}$ & B-290 & 180 & 4.5 & 336 & - & 131 \\
\hline Ni-MOF-74 & Ni/DHBDC & B-290 & 180 & 4.5 & 336 & - & 131 \\
\hline MIL-88A & $\mathrm{Fe} / \mathrm{FA}$ & B-290 & 180 & 4.5 & 336 & - & 131 \\
\hline MIL-88B & $\mathrm{Fe} / 2-\mathrm{NH}_{2}-\mathrm{BDC}$ & B-290 & 180 & 4.5 & 336 & - & 131 \\
\hline IRMOF-3 & $\mathrm{Zn} / 2-\mathrm{NH}_{2}-\mathrm{BDC}$ & B-290 & 180 & 4.5 & 336 & - & 131 \\
\hline ZiF-67 & $\mathrm{Co} / \mathrm{MIM}$ & AF-88 & 180 & 6.7 & & 1861 & 136 \\
\hline ZiF-8 & $\mathrm{Zn} / \mathrm{MIM}$ & B-290 & 180 & 4.5 & 474 & 941 & 131 \\
\hline ZiF-8 & $\mathrm{Zn} / \mathrm{MIM}$ & SD-1000 & 150 & 5 & & 1440 & 134 \\
\hline ZiF-8 & $\mathrm{Zn} / \mathrm{MIM}$ & AF-88 & 180 & 6.7 & & 1634 & 136 \\
\hline $\mathrm{Zn} / \mathrm{Co}-\mathrm{ZiF}$ & $\mathrm{Zn}, \mathrm{Co} / \mathrm{MIM}$ & AF-88 & 180 & 6.7 & & 1746 & 136 \\
\hline MOF & Metal/ligand & Spray dryer & $T_{1} / T_{\text {in }},{ }^{\circ} \mathrm{C}$ & $\begin{array}{l}\text { Feed rate, } \\
\mathrm{mL} \min ^{-1}\end{array}$ & 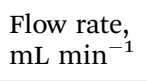 & $S_{\mathrm{BET}}, \mathrm{m}^{2} \mathrm{~g}^{-1}$ & Ref. \\
\hline \multicolumn{8}{|c|}{ Two steps, seed-mediated spray-drying method, dense structures } \\
\hline UiO-66 & $\mathrm{Zr} / \mathrm{BDC}$ & B-290 & $80 / 180$ & 4.5 & 336 & - & 131 \\
\hline UiO-66 & $\mathrm{Zr} / \mathrm{BDC}$ & B-290 & $80 / 220$ & 5 & 300 & - & 137 \\
\hline CID-1 & $\mathrm{Zr} / \mathrm{IP}, \mathrm{BPY}$ & B-290 & $80 / 220$ & 5 & 300 & - & 137 \\
\hline \multicolumn{8}{|c|}{ Two steps, continuous flow spray-drying method, dense structures } \\
\hline $\mathrm{UiO}-66$ & $\mathrm{Zr} / \mathrm{BDC}$ & B-290 & $115 / 180$ & 2.4 & 336 & 1106 & 138 \\
\hline UiO-66 & $\mathrm{Zr} / \mathrm{BDC}$ & B-290 & $115 / 180$ & 2.4 & 336 & 945 & 143 \\
\hline $\mathrm{UiO}-66-\mathrm{NH}_{2}$ & $\mathrm{Zr} / 2-\mathrm{NH}_{2}-\mathrm{BDC}$ & B-290 & $115 / 180$ & 2.4 & 336 & 752 & 138 \\
\hline UiO-66- $\mathrm{NH}_{2}$ & $\mathrm{Zr} / 2-\mathrm{NH}_{2}-\mathrm{BDC}$ & B-290 & $90 / 150$ & 2.4 & 336 & 1036 & 141 \\
\hline $\mathrm{UiO}-66-\mathrm{NO}_{2}$ & $\mathrm{Zr} / 2-\mathrm{NO}_{2}-\mathrm{BDC}$ & B-290 & $115 / 180$ & 2.4 & 336 & 679 & 138 \\
\hline UiO-66-Br & $\mathrm{Zr} / 2-\mathrm{Br}-\mathrm{BDC}$ & B-290 & $115 / 180$ & 2.4 & 336 & 527 & 138 \\
\hline UiO-66- $(\mathrm{OH})_{2}$ & $\mathrm{Zr} / 2,5-(\mathrm{OH})_{2}-\mathrm{BDC}$ & B-290 & $115 / 180$ & 2.4 & 336 & 401 & 138 \\
\hline UiO-66-2,6-NDC & $\mathrm{Zr} / 2,6-\mathrm{NDC}$ & B-290 & $115 / 180$ & 2.4 & 336 & 557 & 138 \\
\hline UiO-66-1,4-NDC & $\mathrm{Zr} / 1,4-\mathrm{NDC}$ & B-290 & $115 / 180$ & 2.4 & 336 & 431 & 138 \\
\hline UiO-66-acetamido & $\mathrm{Zr} / 2,5-(\mathrm{OH})_{2}-\mathrm{BDC}$ & B-290 & $115 / 180$ & 2.4 & 336 & 586 & 138 \\
\hline $\mathrm{UiO}-66-(\mathrm{SH})_{2}$ & $\mathrm{Zr} / 2,5-(\mathrm{SH})_{2}-\mathrm{BDC}$ & B-290 & $115 / 180$ & 2.4 & 336 & 597 & 143 \\
\hline $\mathrm{CeO}_{2} @ \mathrm{UiO}-66$ & $\mathrm{Zr} / \mathrm{BDC}$ & B-290 & $115 / 180$ & 2.4 & 336 & 747 & 143 \\
\hline $\mathrm{CeO}_{2} @ U i O-66-(\mathrm{SH})_{2}$ & $\mathrm{Zr} / 2,5-(\mathrm{SH})_{2}-\mathrm{BDC}$ & B-290 & $115 / 180$ & 2.4 & 336 & 539 & 143 \\
\hline $\mathrm{CeO}_{2} / \mathrm{Fe}_{3} \mathrm{O}_{4} @ \mathrm{UiO}-66-(\mathrm{SH})_{2}$ & $\mathrm{Zr} / 2,5-(\mathrm{SH})_{2}-\mathrm{BDC}$ & B-290 & $115 / 180$ & 2.4 & 336 & - & 143 \\
\hline MIL-100 & $\mathrm{Fe} / \mathrm{BTC}$ & B-290 & $135 / 180$ & 2.4 & 336 & 1039 & 138 \\
\hline$\left[\mathrm{Ni}_{8}(\mathrm{OH})_{4}\left(\mathrm{H}_{2} \mathrm{O}\right)_{2}(\mathrm{~L})_{6}\right]_{n}$ & $\mathrm{Ni} / \mathrm{L}$ & B-290 & $100 / 180$ & 2.4 & 336 & 377 & 138 \\
\hline Zr-fumarate & $\mathrm{Zr} / \mathrm{FA}$ & B-290 & $90 / 150$ & 2.4 & 336 & 664 & 141 \\
\hline
\end{tabular}

Ligand codes: BTC - benzene-1,3,5-tricarboxylic acid; BDC - benzene-1,4-dicarboxylic acid; BPTC - biphenyl-3,3',5,5'-tetracarboxylic acid; BTB 1,3,5-tris(4-carboxyphenyl)benzene; DHBDC - 2,5-dihydroxy-1,4-benzenedicarboxylic acid; FA - fumaric acid; MIM - 2-methyl imidazole; IP isophthalate; BPY - 4,4'-bipyridine; NDC - naphthalenedicarboxylic acid; and L - 1H-pyrazole-4-carboxylic acid. Spray-dryer codes: B-290 - B-290 Mini Spray Dryer (Büchi Labortechnik); SD-1000 - SD-1000 Spray Dryer (Tokyo Rikakikai); and AF-88 - AF-88 Laboratory Spray Dryer (AFIND Scientific Instrument Co. Ltd). “-” not specified.

spray-drying allows the encapsulation of guest molecules in the voids of the formed superstructures. Thus, it was possible to incorporate magnetic iron oxide nanoparticles inside the HKUST-1 hollow microbeads in order to apply the thus formed composite for the removal of fuel contaminants and to easily separate the MOF with a magnet afterwards.

The following study by Carne-Sanchez et al. in $2015^{133}$ showed the possibility to use a spray-dryer to encapsulate MOFs, particularly water-sensitive HKUST-1, into a hydrophobic polymer matrix - polystyrene (PS) - to produce HKUST1@PS composites with improved hydrolytic stability. The feed suspension was composed of the pre-synthesized HKUST-1 dispersed in DMF and a defined portion of PS dissolved in dichloromethane. It was then spray-dried at a feed rate of

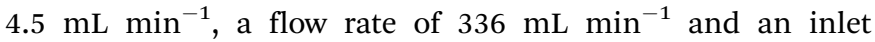
temperature of $130{ }^{\circ} \mathrm{C}$. The resulting product yielded spherical composite microbeads with an average size of $3.6 \pm 1.7 \mu \mathrm{m}$, exhibiting the XRD pattern expected for HKUST-1. The presence of the PS amorphous phase could be assumed by a broad hump on the diffractograms at $2 \theta=15-25^{\circ}$, which increased in intensity with the PS content in the composite. Moreover, the total content of the polymer strongly impacted the textural properties as PS is not adsorptive towards nitrogen. Accordingly, $\mathrm{N}_{2}$ physisorption measurements revealed a decrease in 
$S_{\text {BET }}$ from $1430 \mathrm{~m}^{2} \mathrm{~g}^{-1}$ (pre-synthesized HKUST-1) to $97 \mathrm{~m}^{2} \mathrm{~g}^{-1}$ (HKUST-1@PS composite with 33 wt\% MOF). The optimal composition was found to be the one containing $63 \mathrm{wt} \%$ MOF, which yielded a $S_{\text {BET }}$ of $1202 \mathrm{~m}^{2} \mathrm{~g}^{-1}$, thus preserving the textural properties of the initial MOF powder. Moreover, upon immersion in water overnight, the pre-synthesized HKUST-1 powder was completely degraded. At the same time, the MOF composite preserved its structural and textural integrity both upon immersion in water and upon exposure to humid conditions ( $80 \% \mathrm{RH})$ for one week.

An aqueous spray-drying synthesis of the Zn-imidazole ZIF-8 was done by Tanaka et al. ${ }^{134}$ In a typical synthesis, an aqueous suspension containing $\mathrm{Zn}$-acetate and 2-methylimidazole was spray-dried at $T_{\text {in }}=150{ }^{\circ} \mathrm{C}$ and a feed rate of $5 \mathrm{~mL} \mathrm{~min}^{-1}$. These conditions yielded dense spherical particles with an average size of $3.9 \mu \mathrm{m}$ as confirmed by SEM and TEM. However, the $\mathrm{XRD}$ results suggested the formation of an unknown phase different from that of the original ZIF-8. Moreover, the product poorly adsorbed nitrogen as revealed by $\mathrm{N}_{2}$ sorption measurements. Notably, the authors observed the coordination of dissolved species and therefore the solution turning into a suspension right before spraying. The authors explained this phenomenon as due to the hindrance of crystallization created by acetic acid, a by-product originating from the Zn-precursor. The presence of the acid in the as-synthesized product was demonstrated by means of FTIR spectroscopy and TGA. Accordingly, during the spray-drying process, the as-released acetic acid caused a rearrangement of $\mathrm{Zn}$-(2-methylimidazole) bonds, leading to the amorphization of the final product due to the incomplete coordination of the ligands around the metal. Interestingly, the presence of non-coordinated ligands was similarly evidenced by TGA. However, redispersing the spraydried particles in an alcohol enabled the recrystallization and thus the formation of the targeted ZIF-8 framework. Interestingly, the size of the alcohol molecule influenced the size of the nanocrystals: specifically, the longer the carbon chain the larger the nanocrystals. However, the microbead size remained in the same range. Upon recrystallization, the product yielded an XRD pattern characteristic of ZIF-8 with a $S_{\text {BET }}$ of $1440 \mathrm{~m}^{2} \mathrm{~g}^{-1}$, which is consistent with the results published elsewhere. ${ }^{135}$ Surprisingly, once these ZIF-8 microbeads were redispersed in an alcoholic solution, they undergo a transition from dense to hollow superstructures. Hence, the recrystallization process is fed by gradually dissolving the amorphous byproduct from the surface to the core of the microbeads.

Independently, Chaemchuen et al. ${ }^{136}$ studied the spraydrying of ZIF-8 and its derivatives, the Co-based ZIF-67 and a bimetallic Zn/Co-ZIF. As they faced the same issues as Tanaka et al., ${ }^{134}$ they applied slightly different conditions for spraydrying, namely an inlet temperature $T_{\text {in }}=180{ }^{\circ} \mathrm{C}$ and a feed rate

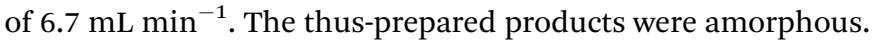
Upon recrystallization in a $\mathrm{MeOH}$ solution and drying, they obtained ZIF-8, ZIF-67 and Zn/Co-ZIF microbeads with the ZIF topology as suggested by the matching XRD patterns. The textural properties studied by $\mathrm{N}_{2}$ physisorption revealed a $S_{\text {BET }}$ of $1634 \mathrm{~m}^{2} \mathrm{~g}^{-1}$ for ZIF-8, $1861 \mathrm{~m}^{2} \mathrm{~g}^{-1}$ for ZIF-67 and
$1746 \mathrm{~m}^{2} \mathrm{~g}^{-1}$ for $\mathrm{Zn} / \mathrm{Co}-\mathrm{ZIF}$. The former is considerably higher as compared to the ZIF-8 microbeads produced by Tanaka et $a .^{134}$ via the same aqueous phase approach. However, this cannot be attributed not only to different spray-drying conditions, but also to a difference in starting concentrations of the ZIF-8 precursors. Last but not least, the spray-dryer machines used for the syntheses come from different manufacturers.

\section{Two step, seed-mediated spray-drying method, dense structures}

Mitsuka et al. ${ }^{137}$ provided an example of a MOF synthesized in 2 consecutive steps. This includes the formation of MOF seeds accomplished by heating up the mixed precursors to a certain temperature $\left(T_{1}\right)$ in a separate vessel, and further spray-drying the thus-formed suspension. Noteworthily, the first step initiates the formation of primary MOF nuclei and the second step, spray-drying, contributes to crystal growth. This approach is generally applied for MOFs consisting of high-nuclearity Secondary Building Units (SBUs), i.e., of metal-clusters composed of multiple metal nuclei such as the UiO family. Following this method, the authors described the successful spray-drying of UiO-66, producing spherical superstructures. The XRD pattern showed reflections matching with the ones of the classical UiO66 synthesized by the solvothermal method. There is, however, a slight peak-broadening observed in the case of the spherical beads. This was attributed to the decreased size (less than $100 \mathrm{~nm}$ ) of the individual UiO-66 nano-particles forming the microbeads, compared to the $500 \mathrm{~nm}$ sized crystals obtained via the solvothermal approach (Fig. 16a). The presence of the typical UiO-66 chemical features was further proven by IR spectroscopy, revealing all bands characteristic of the UiO-66 framework. However, the $\mathrm{N}_{2}$ physisorption performed on both samples indicated a decrease of about $30 \%$ in adsorbed $\mathrm{N}_{2}$ for the spray-dried spherical microbeads. Therefore, some amorphous parts might remain. CID-1, which is a MOF with the formula $\mathrm{Zn}_{2}(\mathrm{ip})_{2}(\mathrm{bpy})_{2}$, was also obtained (see Table 14 ).

\section{Two step, continuous flow spray-drying method, dense structures}

Garzon-Tovar et al. ${ }^{138}$ also reported the spray-drying of a series of MOFs with high-nuclearity. To do so, they combined continuous flow and spray-drying methods (Fig. 16b and c). As in the case of Mitsuka et al., ${ }^{137}$ the former is beneficial to initiate the nucleation step, while the latter favors the crystal growth. Thus, the so-called spray-drying continuous flow-assisted synthesis was applied to produce spherical microbeads of UiO-66 and its derivatives.

In particular, they performed a full study on the production of UiO-66 spherical microbeads. First of all, they found that introducing a continuous flow reactor before the spray-dryer is a crucial step as the application of the spray-dryer alone yielded an amorphous product, as reported by Mitsuka et al. ${ }^{137}$ Moreover, the sole use of a continuous flow reactor without the spray-dryer gave rise to the product with a low yield $(12 \%)$ as well as poor textural properties $\left(S_{\mathrm{BET}}=708 \mathrm{~m}^{2} \mathrm{~g}^{-1}\right)$. Thus, the 

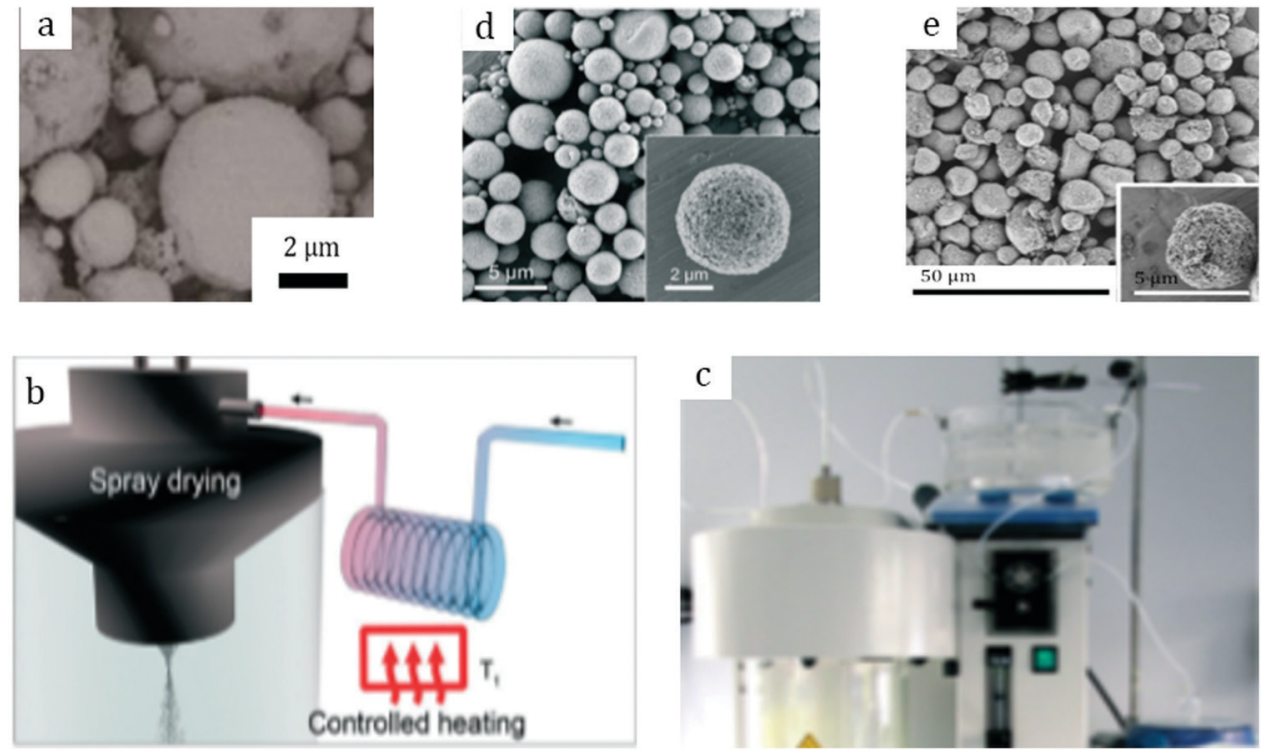

Fig. 16 SEM image of the spray-dried UiO-66 (a) prepared by the seed-mediated spray-drying method. ${ }^{137}$ Copyright 2016 The Chemical Society of Japan. Schematic representation of the spray-drying continuous flow-assisted method (b) and the corresponding photograph (c). ${ }^{138}$ Copyright 2016 Royal Society of Chemistry. UiO-66 (d) prepared by the continuous flow spray-drying method. ${ }^{138}$ Copyright 2016 Royal Society of Chemistry. UiO-66$\mathrm{NH}_{2}$ (e) prepared by the continuous flow spray-drying method. ${ }^{141}$ Copyright 2018 Royal Society of Chemistry.

combination of both was required. The authors further showed that the optimal conditions for the synthesis of UiO-66 and its derivatives included an inlet temperature of $180{ }^{\circ} \mathrm{C}$, a flow rate of $336 \mathrm{~mL} \mathrm{~min}{ }^{-1}$ and a feed rate of $2.4 \mathrm{~mL} \mathrm{~min}^{-1}$. The chosen inlet temperature corresponds to the minimum temperature required for the complete evaporation of the solvent, DMF. The UiO-66 microbeads produced under these conditions yielded dense spherical superstructures with an average size of $4.3 \pm$ $2.6 \mu \mathrm{m}$ composed of individual nanoparticle aggregates (Fig. 16d). XRD confirmed the presence of the UiO-66 structure. In terms of textural properties, the spray-dried microbeads exhibited pronounced porosity with a high $S_{\mathrm{BET}}$ of $1106 \mathrm{~m}^{2} \mathrm{~g}^{-1}$, which is in good agreement with that of UiO-66 produced by classical solvothermal methods $\left(1150-1250 \mathrm{~m}^{2} \mathrm{~g}^{-1}\right) \cdot{ }^{139,140}$

Additionally, lower feed rates of 1.8 and $1.2 \mathrm{~mL} \mathrm{~min}^{-1}$ were attempted and consequently the authors observed clogging inside the flow reactor. Moreover, the products obtained under these conditions exhibited poor textural properties with $S_{\mathrm{BET}}$ of 667 and $687 \mathrm{~m}^{2} \mathrm{~g}^{-1}$, respectively. The authors attributed this phenomenon to the formation of UiO-66 mostly in the flow reactor before it reached the spray-dryer. Importantly, they concluded that the longer the residence time in the flow reactor the higher the content of the MOF formed within it, and therefore the lower the quality of the final product. This explains the lower textural properties of the UiO-66 beads synthesized by Mitsuka et al. ${ }^{137}$ as in their case the initial feed solution was subjected to nucleation for $2 \mathrm{~h}$. Another parameter to highlight is the temperature of the flow reactor, $T_{1}$. GarzonTovar et al. ${ }^{138}$ initiated the nucleation at $115{ }^{\circ} \mathrm{C}$ for as long as $63 \mathrm{~s}$ (which corresponds to $2.4 \mathrm{~mL} \min ^{-1}$ flow rate) and outlined another important observation: a decrease in temperature in the flow reactor led to a decrease in $S_{\mathrm{BET}}$ as well as in the yield of the product. Thus, upon applying $90{ }^{\circ} \mathrm{C}$ they obtained UiO-66 microbeads exhibiting a $S_{\mathrm{BET}}$ of $465 \mathrm{~m}^{2} \mathrm{~g}^{-1}$, which is comparable to the approximately $110 \mathrm{~mL} \mathrm{~g}^{-1}$ at STP (of adsorbed $\mathrm{N}_{2}$ ) obtained by Mitsuka et al. ${ }^{137}$ using the same nucleation temperature. Furthermore, Garzon-Tovar et al. ${ }^{138}$ successfully expanded the spray-drying continuous flowassisted synthesis to other members of the UiO-66 family as well as to other MOFs with high-nuclearity SBUs based on MIL$100(\mathrm{Fe})$ and $\mathrm{Ni}\left[\mathrm{Ni}_{8}(\mathrm{OH})_{4}\left(\mathrm{H}_{2} \mathrm{O}\right)_{2}(\mathrm{~L})_{6}\right]_{n}$ (Table 14).

Avci-Camur et al. ${ }^{141}$ continued exploiting the spray-drying technique for the synthesis of MOFs, targeting the UiO-66 family and more specifically UiO-66- $\mathrm{NH}_{2}$ by the combined continuous-flow spray-drying method under aqueous conditions. For this purpose, the authors used water-soluble $\mathrm{ZrOCl}_{2} \cdot 8 \mathrm{H}_{2} \mathrm{O}$ and 2-aminoterephthalic acid as the metalprecursor and the ligand, respectively. In this work specific stress was given to the use of a modulator, the acetic acid. Generally, the application of monotopic acids such as hydrochloric, formic and acetic acids facilitates the formation/crystallization of the UiO-family of MOFs. ${ }^{142}$ Accordingly, it was shown that an increase in the acid concentration caused significant changes in textural properties. Thus, the UiO-66$\mathrm{NH}_{2}$ prepared with $14 \%$ acetic acid in the feed solution yielded microbeads with a $S_{\mathrm{BET}}$ of $840 \mathrm{~m}^{2} \mathrm{~g}^{-1}$ when spray-dried at $T_{\text {coil }}=$ $90{ }^{\circ} \mathrm{C}, T_{\text {in }}=150{ }^{\circ} \mathrm{C}$, flow rate $=336 \mathrm{~mL} \mathrm{~min}^{-1}$ and feed rate $=$ $2.4 \mathrm{~mL} \mathrm{~min}^{-1}$. However, at elevated (56\%) concentrations of the acid, the $S_{\text {BET }}$ significantly increased up to $1036 \mathrm{~m}^{2} \mathrm{~g}^{-1}$ under the same operating conditions. It should be noted that a further increase $(70 \%)$ in the acid content led to a partial loss in crystallinity viewed as a decrease in reflection intensities in the XRD pattern as well as a loss in $S_{\mathrm{BET}}$ down to $655 \mathrm{~m}^{2} \mathrm{~g}^{-1}$. This suggests a competition between the modulator and the 
ligand for coordination with the metal clusters and therefore subsequent structural collapse upon exceeding occupation of the clusters by the modulator. The optimal acid concentration was found to be $30 \%$. At this value, the spray-dried UiO- $66-\mathrm{NH}_{2}$ yielded microbeads with a size distribution of $4-10 \mu \mathrm{m}$ (Fig. 16e) and exhibiting the UiO-66 structure according to XRD results. Besides, the $S_{\mathrm{BET}}$ value, $1261 \mathrm{~m}^{2} \mathrm{~g}^{-1}$, lies in the range of non-functionalized UiO-66 made via the solvothermal route with DMF, and is much higher than that of the spraydried UiO-66- $\mathrm{NH}_{2}$ prepared by Garzon-Tovar et al. $\left(S_{\mathrm{BET}}=\right.$ $\left.752 \mathrm{~m}^{2} \mathrm{~g}^{-1}\right){ }^{138}$ Finally, the same protocol was applied to the $\mathrm{Zr}$-fumarate MOF. The corresponding information is given in Table 14.

A further study done by Boix et al. ${ }^{143}$ in Maspoch's group led to the incorporation of inorganic nanoparticles into UiO-66 microbeads. The process followed the same sequence of steps, including the formation of primary nuclei in the flow reactor at $115^{\circ} \mathrm{C}$ with a feed rate of $2.4 \mathrm{~mL} \mathrm{~min}^{-1}$, an inlet temperature of $180^{\circ} \mathrm{C}$, and a flow rate of $336 \mathrm{~mL} \mathrm{~min}^{-1}$. The thus-formed UiO66 microbeads exhibited an average size of $1.5 \pm 1.0 \mu \mathrm{m}$ with a crystalline framework corresponding to the UiO-66. Additionally, the beads had a high surface area $\left(S_{\mathrm{BET}}=945 \mathrm{~m}^{2} \mathrm{~g}^{-1}\right)$, which was slightly below than the one obtained by GarzonTovar et al. $\left(S_{\mathrm{BET}}=1106 \mathrm{~m}^{2} \mathrm{~g}^{-1}\right)^{138}$ following the same method. The difference might be attributed to the $\mathrm{Zr}$-source used in each case: zirconium propoxide and zirconium tetrachloride, respectively. Interestingly, the UiO-66 itself was shown to be active towards the adsorption of toxic heavy metals such as $\operatorname{Cr}(\mathrm{vI})$ and $\operatorname{As}(\mathrm{v})$ with removal efficiencies of 99 and $45 \%$, respectively. However, once functionalized with thiol (-SH) groups and doped with $\mathrm{CeO}_{2}$ nanoparticles, it became active and efficient towards heavier metal species including $\mathrm{Cd}(\mathrm{II})$, $\mathrm{Cu}(\mathrm{II}), \mathrm{Pb}$ (II) and $\mathrm{Hg}$ (II) with removal efficiencies of $87,99,99$ and $98 \%$, respectively. Importantly, $\mathrm{CeO}_{2}$-doped UiO-66-(SH) microbeads retained their removal efficiency after 10 adsorption/desorption cycles in a continuous flow column, making them appropriate for further developments as water-purifying adsorbents.

Recently, Boix et al. ${ }^{144}$ extended their study and incorporated $\mathrm{CeO}_{2}$ doped UiO-66 microbeads made via spray drying into millimeter-scale porous polyethersulfone (PES). This was achieved by a dropwise addition of a suspension containing $\mathrm{CeO}_{2} @$ @UiO-66 microspheres and dissolved PES in DMF into a precooled water/ethanolic solution. As a result, PES precipitated, forming a hard shell around the droplets and therefore yielding $\mathrm{CeO}_{2} @ U$ UiO-66@PES granules with an average size of $2 \mathrm{~mm}$. The thus-formed granules with $26 \%$ microbead loading showed a $61 \%$ removal efficiency of an $\mathrm{As}(\mathrm{III})$ and $\mathrm{As}(\mathrm{v})$ mixture as well as removal efficiencies of $75 \% \mathrm{Hg}$ (II) and $72 \% \mathrm{~Pb}$ (II), to name a few. This makes the $\mathrm{CeO}_{2} @ U i O-66 @ P E S$ granules an excellent adsorbent for purifying drinking water from heavy metal pollutants with the potential to be used in the cartridges of home water-filter pitchers.

As a concluding remark, it should be noted that spraydrying, initially intended for drying powdery materials, can be used as a direct synthesis and shaping technique for MOFs. It enables the formation of spherical microbeads with an average size of up to ten micrometers. The microbead size depends on the spray-drying conditions, and mainly on the size of the sprayed droplets at the exit of the nozzle. Generally, this technique allows obtaining highly crystalline MOF microbeads with textural and morphological properties depending on the process conditions and especially the temperature, feed rate and gas flow. Besides, MOFs based on high-nuclearity metal clusters (UiO-66, MIL-100(Fe)) tend to form dense structures, while MOFs with low-nuclearity metal clusters (HKUST-1, ZIF-8) generally form hollow structures. Furthermore, the productivity of the MOF spray-drying process might be improved by implementing a continuous flow of the reaction mixture, with possible space-time yields (STY) of $\sim 20 \mathrm{~kg} \mathrm{~m}^{-3} \mathrm{~d}^{-1}$. ${ }^{138}$

\section{Phase separation methods}

Apart from the shaping techniques considered above, there are other approaches enabling obtaining MOFs with various shapes. In particular and as its name suggests, phase separation relies on using two phases: one containing the MOF, and the other either immiscible or at least with tunable miscibility.

\section{Beads}

One specific phase separation process, sometimes referred to as "molecular gastronomy", relies on using hydrocolloids such as alginate, cellulose, chitosan, starch and other biopolymers together with cross-linking agents: typically, group II metal ions or bases. Generally, when a cross-linking agent comes in contact with an aqueous slurry of a biopolymer and the pristine MOF, it precipitates the biopolymer which entraps the MOF particles and serves as the matrix (Fig. 17a). This approach was first described by Spjelkavik et al. ${ }^{145}$ who formulated Ni-based CPO-27 into spherical beads of a few millimeters. More precisely, they used either a solution of $\mathrm{CaCl}_{2}$ or $\mathrm{BaCl}_{2}$ as the cross-linking agent, which was added dropwise into an aqueous CPO-27/alginate slurry. Eventually, after a certain time, the spheres were isolated, washed and dried to yield beads with MOF contents up to $90 \mathrm{wt} \%$. Besides, the authors found that the crystal structure remained intact by the end of this procedure. In addition, they outlined the importance of the gelation (residence) time. Thus, the longer the gelation time, the more drastic the loss in surface area for the final beads. Therefore, the authors suggested $0.5 \mathrm{~h}$ to be a fair compromise between rigidifying the objects and maintaining the accessibility to the pores of $\mathrm{CPO}-27$.

Lee et $a l .{ }^{146}$ systematically studied the shaping of UiO-66 into spherical beads by the "alginate" method. Namely, they addressed the impact of different parameters such as the MOF content, gelation time, and activation temperature on the properties of the final beads. Thus, they found that the alginate had no effect on the crystal structure of the MOF. Besides, the loss in specific surface area upon shaping was within the expected range and consistent with the alginate content. Thus, for the beads with polymer contents of 2.4 and $4.8 \mathrm{wt} \%$, the 

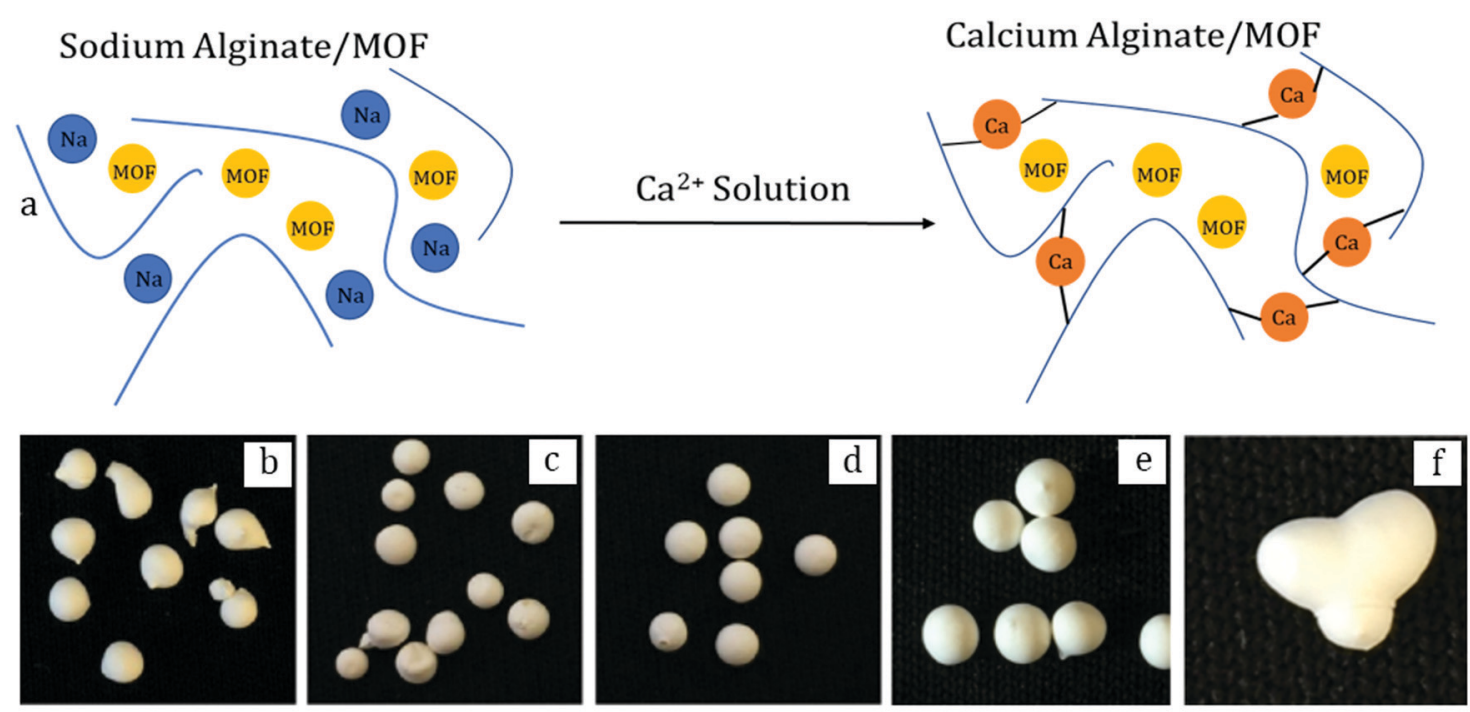

g
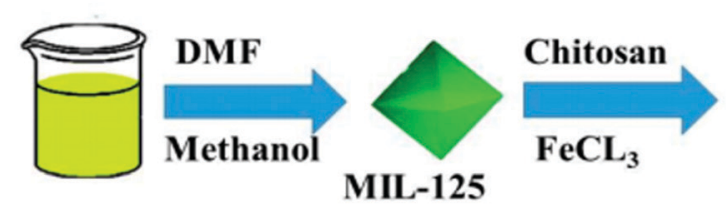

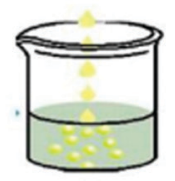

$\mathrm{Na}_{5} \mathbf{P}_{3} \mathrm{O}_{10}$ solution

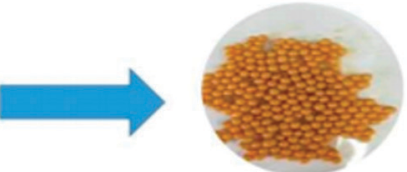

MIL-125-CS

Fig. 17 Schematic representation of the "molecular gastronomy" method (a). UiO-66 beads (b-f) prepared by Lee et al. ${ }^{146}$ showing the effect of the concentration of the gelation solution on the final shape of the beads: $b-0.2 \%(w / v) ; c-0.5 \%(w / v) ; d-2.0 \%(w / v)$; and e and $f-10 \%(w / v)$. Copyright 2020 Elsevier. Preparation of MIL-125(Ti) beads (g) with chitosan as a binding biopolymer. ${ }^{149}$ Copyright 2018 MDPI.

decreases in $S_{\mathrm{BET}}$ were 4.0 and $6.5 \%$, respectively, relative to the parent UiO-66 with a $S_{\mathrm{BET}}$ of $1241 \mathrm{~m}^{2} \mathrm{~g}^{-1}$. Interestingly, it was shown under these conditions that the gelation time had no significant impact on the textural properties as the beads with 3 min and $14 \mathrm{~h}$ of gelation yielded comparable $\mathrm{N}_{2}$ isotherms. Moreover, the final shape of the beads was dictated by the concentration of $\mathrm{CaCl}_{2}$ in the gelation solution (Fig. 17b-f). Importantly, the optimal activation temperature was found to be in the range of $120-135{ }^{\circ} \mathrm{C}$ to avoid the biopolymer degradation.

Farrusseng et al. ${ }^{147}$ studied the shaping of the $\mathrm{Al}(\mathrm{OH})$ fumarate MOF for the heat-pump application. They found that the "alginate" method had little effect on the MOF textural properties, leading to losses in specific surface area comparable to the initial alginate content, as reported by Lee et al. As an example, beads with $\sim 12$ wt $\%$ alginate demonstrated a $10 \%$ reduction of $S_{\mathrm{BET}}$ as compared to the pristine MOF (997 vs. $1098 \mathrm{~m}^{2} \mathrm{~g}^{-1}$ ). Consequently, the water sorption capacity of the MOF-based beads was reduced by the same extent, suggesting no pore blocking caused by the polymer. Importantly, the alginate content in the beads also had no significant impact on mass and heat transfers.

Yang et al. ${ }^{148}$ modified the "alginate" method by adding an extra cross-linker agent: polyacrylic acid (PAA). Interestingly, this polymer presents a large molecular weight and rapidly polymerizes, limiting the polymer's ability to diffuse into the
MOF's porosity. This procedure was applied to numerous MOFs, including MIL-127(Fe), MIL-101(Cr), HKUST-1, UiO-66, ZIF-8, and Fe-BTC. All beads presented MOF loadings higher than $84.9 \mathrm{wt} \%$ and almost preserved BET surface areas (normalized by the MOF loading), except for HKUST-1, which partially collapsed due to its exposure to strongly acidic conditions $(\mathrm{pH}=$ 2.7). SEM-EDX analysis on the cross-section of a bead concluded that the MOF crystals were homogeneously distributed. Computational modeling was performed to unveil the chemical interactions between alginate, PAA, and $\mathrm{Ca}^{2+}$. Spontaneous binding of carboxyl and/or hydroxyl groups to $\mathrm{Ca}^{2+}$ was observed. Moreover, $a b$ initio simulations between MIL$127(\mathrm{Fe})$, a representative MOF, and both polymers unveiled that $\mathrm{Ca}^{2+}$ would bind to the free carboxylic groups of the ligands on the surfaces of the MOFs and the polymers, while polar carboxyl and hydroxyl groups of PAA and alginate would bind to the metal sites of MIL-127(Fe). This was experimentally verified by FT-IR and Raman spectroscopies. According to the authors, improved mechanical stability of the composite beads should be obtained, but it was not measured.

Finally, Yang et al. ${ }^{148}$ prepared polydopamine-grafted FeBTC-based beads for the extraction of lead from Rhône river water (600 ppb). Under continuous flow, one gram of beads could reduce the concentration of 10.8 liters of water below the EPA limit of $15 \mathrm{ppb}$. Of note, most commercial adsorbents were poorly performing below $150 \mathrm{ppb}$ due to the presence of 
competitive ions. Thus, the authors concluded that the composite beads were selective for $\mathrm{Pb}^{2+}$ over less toxic ions. Moreover, they could be readily regenerated in the presence of EDTA.

Liang et al. ${ }^{149}$ studied the shaping of a Ti-based MIL-125 MOF with chitosan as a binding biopolymer into spherical beads. They first mixed chitosan and an $\mathrm{FeCl}_{3}$ solution, followed by the addition of the MOF. Once well mixed, a 3\% $\mathrm{Na}_{5} \mathrm{P}_{3} \mathrm{O}_{10}$ solution was added dropwise to initiate the crosslinking step (Fig. 17g). The thus-formed beads were recovered, washed and dried. The authors showed that such a formulation had no impact on the crystal structure nor the framework composition as confirmed by XRD, FTIR spectroscopy and XPS analyses. Therefore, the beads exhibited a consequent capacity for the removal of $\mathrm{Pb}$ (II) species, with only an $\sim 12 \%$ decrease in efficiency (from 100 to $88 \mathrm{mg} \mathrm{g}^{-1}$ ) after five consecutive cycles.

Chitosan was also used by Omer et al. ${ }^{150}$ to produce magnetic ZIF-67 beads with a diameter of $1.65 \mathrm{~mm}$. For that, they dispersed the as-made ZIF-67 crystallites and $\mathrm{Fe}_{3} \mathrm{O}_{4}$ nanoparticles in water under sonication. The suspension was then mixed into a solution of chitosan dissolved in acetic acid, and the resulting mixture was added dropwise into $\mathrm{NaOH}$ solution, and aged for 15 minutes prior to the recovery of the beads using a magnet. The FT-IR spectrum of the beads revealed broad bands at 1062 and $3441 \mathrm{~cm}^{-1}$, attributed to the stretching vibration of $\mathrm{C}-\mathrm{N}$ and $\mathrm{O}-\mathrm{H}$ from chitosan, respectively. The presence of $\mathrm{Fe}_{3} \mathrm{O}_{4}$ and ZIF-67 was also attested to by broad bands at 557 and $1405 \mathrm{~cm}^{-1}$ (Fe-O stretching), and $413 \mathrm{~cm}^{-1}$ (Co-N stretching), respectively. A soft-ferromagnetic behavior was observed using a vibrating sample magnetometer, while a significant decrease in the saturation magnetization was observed and attributed to the presence of non-magnetic ZIF67 and chitosan. SEM images and digital micrographs showed that the beads were irregular and presented a core-shell structure, with ZIF-67 particles mostly located at the core. A type IV isotherm was measured by $\mathrm{N}_{2}$ physisorption, with an $\mathrm{H} 4$ hysteresis loop typically characterizing the presence of mesopores. The BET surface area of such composite beads reached $221 \mathrm{~m}^{2} \mathrm{~g}^{-1}$ : for comparison, ZIF-67 powders generally present BET surface areas above $1100 \mathrm{~m}^{2} \mathrm{~g}^{-1}$. Hence, partial pore blockage by the chitosan was suspected. Nonetheless, the composite beads presented a higher $\mathrm{Cr}(\mathrm{vI})$ adsorption capacity $\left(71 \mathrm{mg} \mathrm{g}^{-1}\right)$ than the MOF itself $\left(45 \mathrm{mg} \mathrm{g}^{-1}\right)$. The composite beads' reusability was evaluated and, after the seventh cycle, the capacity of the beads only decreased from 71 to $61 \mathrm{mg} \mathrm{g}^{-1}$. XPS spectroscopy was performed to propose an adsorption mechanism in three steps: 1 - protonation of the amino groups of the chitosan and coulombic interaction between the positively charged beads and the anionic $\operatorname{Cr}(\mathrm{vI}) ; 2$ - reduction of $\mathrm{Cr}$ (VI) to $\mathrm{Cr}(\mathrm{III})$ by the electron-donor groups; and 3 - formation of coordinate-covalent bonds between $\mathrm{Cr}(\mathrm{III})$ and the composite beads.

Besides biopolymers, other types of polymers can lead to the formation of beads. For instance, HKUST-1-polyacrylonitrile (PAN) composite beads were formed by Riley et al.,${ }^{151}$ by adding dropwise a DMSO mixture containing dissolved PAN and dispersed HKUST-1 crystallites into an aqueous solution. The MOF loading was varied from 10 to $90 \mathrm{wt} \%$ (on a dry basis). The final beads presented a size distribution of $3.17 \pm 0.26 \mathrm{~mm}$, and the MOF crystals were evenly distributed within the beads. X-ray computed tomography and SEM on cross-sectioned beads revealed the presence of 0.5-1 $\mu \mathrm{m}$ macropores homogeneously distributed. Thus, PAN allowed obtaining hierarchically porous beads following phase separation. The maximal compressive strength of 90 wt $\%$ HKUST- 1 beads reached 18 MPa. Finally, the beads were used for xenon capture, comparatively to the powder. The 90 wt $\%$ HKUST- 1 beads presented a capacity $30 \%$ lower than that of the powder, which might be due to partial pore blockage by the PAN. Finally, the beads were gamma irradiated up to $100 \mathrm{kGy}$ and the MOF retained most of its crystallinity, making it an interesting candidate for further demonstration tests.

Park et $a .^{152}$ shaped a diamine-functionalized epn$\mathrm{Mg}_{2}$ (dobpdc) MOF (dobpdc $=4,4^{\prime}$-dioxido-3,3'-biphenyldicarboxylate, epn $=1$-ethylpropane-1,3-diamine) into beads using poly(vinylidene fluoride) (PVDF). For this, the pristine MOF powder was dispersed in a DMF solution containing the polymer dissolved. Then, the slurry was added dropwise through a syringe into a water/ethanol $(5: 1, \mathrm{v} / \mathrm{v})$ mixture. Spherical beads of a few millimeters were rapidly formed and subsequently recovered, washed with $\mathrm{MeOH}$ and dried. Thereafter, the beads were immersed in an epn solution in toluene to graft the diamine precursor onto the MOF. Upon this multi-step process, the structural integrity of the MOF crystals was preserved as observed by XRD. The beads containing $60 \mathrm{wt} \%$ MOF showed a $\mathrm{CO}_{2}$ adsorption capacity of $1.48 \mathrm{mmol} \mathrm{g}^{-1}$ at $1000 \mathrm{ppm}$ and $25{ }^{\circ} \mathrm{C}$, as compared to $2.49 \mathrm{mmol} \mathrm{g}^{-1}$ for the pristine MOF functionalized with epn. This value agrees with the polymer content in the beads and represents a $41 \%$ loss. Moreover, the beads demonstrated fair stability towards $\mathrm{CO}_{2}$ adsorption under humid conditions $(60 \% \mathrm{RH})$, losing only $22 \%$ of their efficiency after regeneration at $70{ }^{\circ} \mathrm{C}$ for $1 \mathrm{~h}$, and outperforming the powder counterpart, which lost $72 \%$ of its capacity after regeneration. This highlights an enhanced stability of the composites under humid conditions due to the presence of the hydrophobic polymer.

Another type of phase separation technique relies on the use of freezing solvents. Gökpinar et $a l^{153}$ studied the freezegranulation of a series of MOFs: two Zr-based (UiO-66 and Zrfumarate) and two Al-based (MIL-160 and Al-fumarate). In any case, the parent MOF powder was mixed with the PVA binder pre-dissolved in water ( $80 \mathrm{wt} \%$ MOF dry content). The thusformed suspension was further added dropwise through a syringe into liquid nitrogen. The pellets were collected and dried after solvent-exchange. This procedure enabled spherical MOF/PVA beads and had no impact on the crystal structure as well as on textural properties. Thus, MOF/PVA spheres retained $74-99 \%$ of the MOF original surface area (with respect to the MOF content). Besides, the beads exhibited macropores of a few microns. Importantly, there was a slight increase in water uptake shown by the Zr-based MOFs upon shaping (113\% for Zr-fumarate/PVA and 103\% for UiO-66/PVA beads). This was 
attributed to the generation of new defect sites upon freezegranulation.

\section{Monoliths}

While phase separation is mostly applied to prepare MOFbased composite beads, monoliths can also be obtained using similar approaches. In particular, Hasturk et al. ${ }^{154}$ shaped $\mathrm{Al}-$ fumarate and Cr-based MIL-100 into mechanically stable monoliths. For this, they first dissolved some PVA in water, prior to the addition of the MOF powder. Further acetone was added dropwise to the MOF/PVA suspension, and the thusformed two phase mixture was left still for $48 \mathrm{~h}$ until complete separation. During this period, acetone gradually penetrated the polymer-enriched phase and initiated its gelation. Eventually, it was washed with fresh acetone and dried in a vacuum at room temperature, thus yielding monoliths with up to $80 \mathrm{wt} \%$ MOF. This procedure was shown to have no effect on the crystal structure nor on the textural properties.

Following spinodal decomposition, which is also a phase separation method, Hara et al. ${ }^{155}$ prepared UiO-66_NH ${ }_{2}$-based monolithic materials with a trimodal pore structure. For that, all MOF precursors were dissolved into DMF along with poly(propylene glycol) (PPG) at $60{ }^{\circ} \mathrm{C}$, and the clear solution was sealed in a hydrophobic glass tube kept at $80{ }^{\circ} \mathrm{C}$. After 12 hours, hydrophilic UiO-66_NH $\mathrm{NH}_{2}$ MOF mismatched growth occurred, as well as phase separation with the hydrophobic PPG. After washing with solvent, PPG was evacuated from the monolithic solid, leading to the formation of macropores whose diameter, between 0.9 and $1.8 \mu \mathrm{m}$, can be controlled by the amount of PPG. The XRD patterns displayed a few broad reflections, with $2 \theta$ positions comparable to those of the simulated UiO-66. The structural properties of the MOF were proven by FT-IR spectroscopy, yielding a spectrum comparable to that of standard UiO-66_NH $\mathrm{NH}_{2}$ powder. All samples presented specific surface areas between 712 and $749 \mathrm{~m}^{2} \mathrm{~g}^{-1}$, further underlining the presence of a microporous network, while interparticular mesoporosity could also be deduced from $\mathrm{N}_{2}$ sorption isotherms at higher relative pressure. Indeed, the TEM images showed particles with sizes below $50 \mathrm{~nm}$. Uniaxial compression tests demonstrated that these monoliths presented a maximal compressive strength of $2.5 \mathrm{MPa}$. Interestingly, the authors showed that addition of acetic acid, a known modulator accelerating the crystallization, allowed obtaining larger mesopores. Alternatively, a post-shaping solvothermal treatment also allowed controlling the final size of the mesopores following the secondary growth of the MOF crystals.

In 2014 Ahmed et al. ${ }^{156}$ proposed a different method for MOF shaping based on controlled freezing. According to it, a MOF powder in suspension can be shaped into monoliths upon controlled freezing of the solvent with its subsequent elimination via freeze-drying. The authors applied this methodology to obtain Cu-based HKUST-1 monoliths. For this, the MOF precursors were dissolved in DMSO and left for $24 \mathrm{~h}$ at $80{ }^{\circ} \mathrm{C}$. After that, the solution was frozen in liquid nitrogen for $1 \mathrm{~min}$ and placed into a freeze-dryer to sublime the solvent. This procedure yielded highly crystalline HKUST-1 monoliths as confirmed by XRD. Moreover, the specific surface area was $870 \mathrm{~m}^{2} \mathrm{~g}^{-1}$ with characteristics of both micropores and mesopores, as visible from the $\mathrm{N}_{2}$ physisorption isotherms. Additionally, as shown by $\mathrm{Hg}$ intrusion, the monoliths exhibited macropores with diameters around 0.4 and $10 \mu \mathrm{m}$. Importantly, these macropores generated upon ice-templating were oriented in one particular direction due to the orientational growth of ice crystals during freezing. Lastly, the authors showed that the size of these macropores could be varied by altering the freezing temperature. Thus, upon freezing at $5{ }^{\circ} \mathrm{C}$ the macropores were two times bigger $(\sim 50 \mu \mathrm{m})$ than the macropores generated upon freezing at -80 and $-20{ }^{\circ} \mathrm{C}(32$ and $25 \mu \mathrm{m}$, respectively).

Fu et $_{\text {al. }}{ }^{157}$ studied the preparation of ice-templated composite monoliths based on UiO-66 MOF and chitosan for wastewater treatment. In a typical procedure, the parent UiO- 66 was added into a solution containing pre-dissolved chitosan with $1: 1$ and $1: 2$ weight ratios. After that, part of the suspension was immersed in liquid nitrogen for 5 min followed by freezedrying for $48 \mathrm{~h}$. The results suggest that the thus-formed monoliths preserved the crystal structure of the parent UiO66 , with a surface area in accordance with the MOF ratio $\left(339 \mathrm{~m}^{2} \mathrm{~g}^{-1}\right.$ for the UiO-66/chitosan composite with a $1: 2$ weight ratio, as compared to a $S_{\text {BET }}$ of $1034 \mathrm{~m}^{2} \mathrm{~g}^{-1}$ for the parent UiO-66). However, the monoliths were stated to undergo disintegration upon immersion in an aqueous solution of methylchlorophenoxypropionic acid (a toxic pesticide). This was attributed to the presence of residual acetic acid used in the preparation step. Therefore, the monoliths were washed with $\mathrm{NaOH}$ solution to remove the acid and overcome the disintegration. Interestingly, the results indicated a pesticide removal capacity of the UiO-66/chitosan $(1: 2)$ composite as high as that of the parent UiO-66 ( 34 and $36 \mathrm{mg} \mathrm{g}^{-1}$, respectively). This is due to the contribution of the chitosan, which also presented a consequent pesticide removal capacity $\left(27 \mathrm{mg} \mathrm{g}^{-1}\right)$.

Other studies by Fu et al. ${ }^{158,159}$ include the further pyrolysis of ice-templated MOF/polymers composites to produce hierarchically porous carbon materials. Thus, they formed carbon/ $\mathrm{ZrO}_{2}$ composites upon the carbonization of ice-templated UiO66/poly(4-styrenesulfonate) composites at $800{ }^{\circ} \mathrm{C}$ under Ar. ${ }^{159}$ Although a drastic loss in surface area was observed upon pyrolysis $\left(368 \mathrm{~m}^{2} \mathrm{~g}^{-1}\right.$ vs. $1034 \mathrm{~m}^{2} \mathrm{~g}^{-1}$ for the parent UiO-66), the newly-formed material presented an interesting hierarchical porosity composed of micropores inherited from the MOF, mesopores generated due to interparticular voids, and macropores generated by the ice-templating process. Moreover, metal oxide-free porous carbons were obtained by replacing UiO-66 with ZIF-8, and subsequently carbonizing ice-templated ZIF-8/ polyacrylonitrile (PAN) monoliths at $1000{ }^{\circ} \mathrm{C}$ under Ar. The textural properties of the thus-formed monoliths were also described by micro-meso-macroporous features, with a $S_{\mathrm{BET}}$ of $530 \mathrm{~m}^{2} \mathrm{~g}^{-1}$.

Tian et al. ${ }^{160}$ showed the possibility to form ZIF-8 monoliths without using binders nor high pressures. For this, they immersed a newly-formed ZIF-8 powder into an ethanolic solution containing dissolved precursors (Zn-nitrate and 
methylimidazole), and the solid product was recovered via centrifugation. The authors outlined the importance of drying the solid at room temperature so that it retained its monolithic shape. Besides, when extra amounts of precursors were added, mechanically stable monoliths were formed due to the extension of polymerization reactions. The mechanical resistance was assessed by measuring the elastic modulus (7 GPa) and hardness ( $0.6 \mathrm{GPa})$ by nanoindentation. The thus-formed monoliths retained the original crystal structure of ZIF-8 as well as high specific surface area, up to $1395 \mathrm{~m}^{2} \mathrm{~g}^{-1}$.

To conclude, there is a plethora of studies which show the possibility to produce robust MOF objects using (bio)polymers as the cross-linking/binding agents as well as through sublimation or phase transition phenomena. These so-called "phase separation" methods enable the shaping of MOF powders into differently objects - mostly beads or monoliths - without using specific equipment; with the exception being freeze-drying for which, as its name suggests, a freeze-dryer is needed. Interestingly, the final produced MOF composites present high BET surface areas, in line with their weight composition, as well as good mechanical stability.

\section{Conclusion and perspectives}

The outstanding properties of Metal-Organic Frameworks rapidly grabbed the interest of both academics and industrialists, and an abundant body of literature covering all related aspects is available nowadays. On the specific aspect of shaping, since the late $2000 \mathrm{~s}$ an increasing number of articles can be found, first based on unidirectional compression which is the most straightforward technology for shaping powders, then on other classical technologies (extrusion, granulation). Nowadays, all available techniques can be employed to shape MOFbased materials, each with specific strengths and weaknesses that have been underlined in this review.

Pelletization has been regarded for long as the "reference" technology for comparing shaping techniques. Obviously, when applying forces in the range of the ones applied industrially to zeolites and activated carbons, the MOF structures typically collapse which directly impact their final performances. However, recent developments show that when applying mild compression, along with the presence of a scaffolding compound (solvent or other), the collapsing is greatly limited. Moreover, binderless pelletization is possible owing to MOF crystals' binding ability. Therefore, pelletization offers some of the best compromises between mechanical stability, and volumetric and gravimetric uptakes, making it a leading technique for mobility applications. To further limit the effect of compression on MOFs while reaching high mechanical resistance, isostatic compression could be advantageously used.

Extrusion is another classical technique which is especially used to produce extrudates and honeycombs for catalytic converters. When it is applied to MOFs, limited impact on the structural and textural properties can be observed for most MOFs, due to lower pressures and shear forces applied.
Extrusion requires, however, finely controlling the formulation and related rheological properties of the extruded paste. Advantageously, extrusion can also be used for the direct preparation of MOF objects starting from precursors (reactive extrusion). The latter is of particular interest as it allows limiting or avoiding completely the toxic solvents traditionally used for the synthesis of MOF powders. At the same time, reactive extrusion implies a continuous process with high potential space time yields. While this approach might not be applicable to all MOF structures, the reactive extrusion presents several advantages over more conventional methods such as solvo/ hydrothermal or microwave-assisted syntheses of MOFs. On the other hand, these conventional methods remain better in terms of obtained crystallinity and surface area for most MOF structures.

Since 2013, another technique for both forming and shaping MOFs has become popular. Based on the principle of spraydrying, which is the pulverization of a suspension in a heated environment for fast drying, microbeads of calibrated diameters can be obtained starting from precursors. Herein, the fast reaction rates within the nanodroplets of suspensions, acting like nanoreactors, allow obtaining highly crystalline MOF particles which agglomerate into hollow or dense fluidized powder.

Granulation is the last industrially-mature technology reviewed herein, and allows producing millimeter-sized grains. Two types of granulation techniques are typically discussed: wet granulation, when powders are aggregated in a high-shear rate mixer in the presence of a solvent; and dry granulation, when grains are obtained from a previously shaped object either mildly crushed and sieved, or spheronized. Due to higher stresses applied, the dry granulation implies more severe losses in the initial physicochemical properties of the MOFs, while the wet granulation has a less pronounced effect and therefore might be more adequate. Especially, replacing water with another solvent with a lower surface tension is highly beneficial.

Starting from 2015, the additive fabrication techniques, also called 3D printing, have been widely applied to a large selection of MOF structures to produce precisely designed objects. Among the various technologies available, SLS and DLP might not be the most adequate as, contrarily to ceramics and metals, MOFs cannot handle high temperatures and therefore are partially decomposed under laser irradiation. Moreover, the photopolymerizable resins used in DLP cannot be eliminated by thermal decomposition, thus blocking the porosity and largely affecting the performances of the MOFs. Therefore DIW, which can be viewed as the three-dimensional extrusion of a shear-thinning MOF-based paste, has been largely adopted. Highly interesting results have been obtained in terms of gravimetric uptake and mechanical stability, and therefore this technology is promising for atypical applications necessitating unconventional shapes. These advances should be consolidated with the development of new setups with increased printing accuracy, smaller nozzle diameter and improved paste/ink formulations. 
Finally, other less-popular techniques have been successfully applied for shaping MOFs, among which have been reviewed the so-called molecular gastronomy, ice-templating (also called freeze-casting), and phase separation (also called spinodal decomposition). These three techniques presented very low impact on the physicochemical properties of the MOFs applied and are therefore worth investigating more in detail. It should be noted, however, that ice-templating and phase separation both involve the creation of a second level of porosity macrosized ( $>50 \mathrm{~nm}$ ) following the replication of ice crystals and polymers, respectively.

In order to better understand the failing mechanisms and improve the resistance of the final objects produced while not sacrificing the physicochemical properties brought about by the MOFs, the modeling of the porous microstructure would be highly beneficial. Modeling software packages based on either finite elements or discrete elements, and coupled with X-ray tomography observations and different mechanical characterization studies such as micro-indentation, have been applied to porous catalysts and could be advantageously used as well on these objects.

While keeping in mind the final application, for energy storage, fixed bed reactors, or toxic gas masks just to name a few, specific shaping methods have been developed and most prerequisites can be met nowadays in order to bring MOFs to the forefront of highly performing materials. However, to be durably implemented, besides cutting down their cost and carefully evaluating their environmental and health impacts, efforts are still needed to reach or even exceed the mechanical resistance of inorganic-based objects. This would ensure industrial acceptance, the way zeolites were broadly introduced in refining and petrochemical processes in the last century.

Lastly, comparative data on the specifics and general performance of the conventional shaping techniques are given in Table 15.

\section{Conflicts of interest}

There are no conflicts to declare.

\section{References}

1 O. M. Yaghi and H. Li, Hydrothermal Synthesis of a MetalOrganic Framework Containing Large Rectangular Channels, J. Am. Chem. Soc., 1995, 117, 10401-10402, DOI: 10.1021/ja00146a033.

2 O. M. Yaghi, M. O'Keefe, N. W. Ockwig, H. K. Chae, M. Eddaoudi and J. Kim, Reticular synthesis and the design of new materials, Nature, 2003, 423, 705-714, DOI: 10.1038/nature01650.

3 S. S.-Y. Chui, S. M.-F. Lo, J. P. H. Charmant, A. G. Orpen and I. D. Williams, A Chemically Functionalizable Nanoporous Material $\left[\mathrm{Cu}_{3}(\mathrm{TMA})_{2}\left(\mathrm{H}_{2} \mathrm{O}\right)_{3}\right] \mathrm{n}$, Science, 1999, 283, 1148-1151, DOI: 10.1126/science.283.5405.1148. 
4 J. H. Cavka, S. Jakobsen, U. Olsbye, N. Guillou, C. Lamberti, S. Bordiga and K. P. Lillerud, A New Zirconium Inorganic Building Brick Forming Metal Organic Frameworks with Exceptional Stability, J. Am. Chem. Soc., 2008, 130, 13850-13851, DOI: 10.1021/ja8057953.

5 J. Y. Choi, R. Huang, F. J. Uribe-romo, H. K. Chae, K. S. Park, Z. Ni, A. P. Co, M. O. Keeffe and O. M. Yaghi, Exceptional chemical and thermal stability of zeolitic imidazolate frameworks, Proc. Natl. Acad. Sci. U. S. A., 2006, 103, 10186-10191, DOI: 10.1073/pnas.0602439103.

6 G. Férey, C. Mellot-Draznieks, C. Serre, F. Millange, J. Dutour, S. Surblé and I. Margiolaki, A Chromium Terephthalate-Based Solid with Unusually Large Pore Volumes and Surface Area, Science, 2005, 309, 2040-2042, DOI: $10.1126 /$ science.1116275.

7 Z. R. Herm, R. Krishna and J. R. Long, $\mathrm{CO}_{2} / \mathrm{CH}_{4}, \mathrm{CH}_{4} / \mathrm{H}_{2}$ and $\mathrm{CO}_{2} / \mathrm{CH}_{4} / \mathrm{H}_{2}$ separations at high pressures using $\mathrm{Mg}_{2}$ (dobdc), Microporous Mesoporous Mater., 2012, 151, 481-487, DOI: 10.1016/j.micromeso.2011.09.004.

8 A. Dhakshinamoorthy, Z. Li and H. Garcia, Catalysis and photocatalysis by metal organic frameworks, Chem. Soc. Rev., 2018, 47, 8134-8172, DOI: 10.1039/C8CS00256H.

9 L. Wang, M. Zheng and Z. Xie, Nanoscale metal-organic frameworks for drug delivery: a conventional platform with new promise, J. Mater. Chem. B, 2018, 6, 707-717, DOI: 10.1039/C7TB02970E.

10 X. Fang, B. Zong and S. Mao, Metal-Organic Framework-Based Sensors for Environmental Contaminant Sensing, Nano-Micro Lett., 2018, 10, 64, DOI: 10.1007/s40820-018-0218-0.

11 M. Mon, R. Bruno, J. Ferrando-Soria, D. Armentano and E. Pardo, Metal-organic framework technologies for water remediation: towards a sustainable ecosystem, J. Mater. Chem. A, 2018, 6, 4912-4947, DOI: 10.1039/C8TA00264A.

12 Y. Zhao, Z. Song, X. Li, Q. Sun, N. Cheng, S. Lawes and $\mathrm{X}$. Sun, Metal organic frameworks for energy storage and conversion, Energy Storage Mater., 2016, 2, 35-62, DOI: 10.1016/j.ensm.2015.11.005.

13 Y. V. Kaneti, J. Tang, R. R. Salunkhe, X. Jiang, A. Yu, K. C. Wu and Y. Yamauchi, Nanoarchitectured Design of Porous Materials and Nanocomposites from MetalOrganic Frameworks, Adv. Mater., 2017, 29, 1604898, DOI: 10.1002/adma.201604898.

14 Q. Wang and D. Astruc, State of the Art and Prospects in Metal-Organic Framework (MOF) - Based and MOFDerived Nanocatalysis, Chem. Rev., 2020, 120, 1438-1511, DOI: 10.1021/acs.chemrev.9b00223.

15 R. R. Salunkhe, Y. V. Kaneti and Y. Yamauchi, Metal-Organic Framework-Derived Nanoporous Metal Oxides toward Supercapacitor Applications: Progress and Prospects, ACS Nano, 2017, 11, 5293-5308, DOI: 10.1021/ acsnano.7b02796.

16 M. Zhong, L. Kong, N. Li, Y. Liu, J. Zhu and X. Bu, Synthesis of MOF-derived nanostructures and their applications as anodes in lithium and sodium ion batteries, Coord. Chem. Rev., 2019, 388, 172-201, DOI: 10.1016/ j.ccr.2019.02.029.
17 C. Wang, Y. V. Kaneti, Y. Bando, J. Lin, C. Liu, J. Li and Y. Yamauchi, Metal-organic framework-derived onedimensional porous or hollow carbon-based nanofibers for energy storage and conversion, Mater. Horiz., 2018, 5, 394-407, DOI: 10.1039/C8MH00133B.

18 C. Perego and P. Villa, Catalyst preparation methods, Catal. Today, 1997, 34, 281-305, DOI: 10.1016/S09205861(96)00055-7.

19 F. Lorignon, A. Gossard and M. Carboni, Hierarchically porous monolithic MOFs: An ongoing challenge for industrial-scale effluent treatment, Chem. Eng. J., 2020, 393, 124765, DOI: 10.1016/j.cej.2020.124765.

20 R. Bingre, B. Louis and P. Nguyen, An Overview on Zeolite Shaping Technology and Solutions to Overcome Diffusion Limitations, Catalysts, 2018, 8, 163, DOI: 10.3390/ catal8040163.

21 R. V. Jasra, B. Tyagi, Y. M. Badheka, V. N. Choudary and T. S. G. Bhat, Effect of Clay Binder on Sorption and Catalytic Properties of Zeolite Pellets, Ind. Eng. Chem. Res., 2003, 42, 3263-3272, DOI: 10.1021/ie0109531.

22 G. T. Whiting, A. D. Chowdhury, R. Oord, P. Paalanen and B. M. Weckhuysen, The curious case of zeolite - clay/ binder interactions and their consequences for catalyst preparation, Faraday Discuss, 2016, 188, 369-386, DOI: 10.1039/c5fd00200a.

23 V. Finsy, L. Ma, L. Alaerts, D. E. De Vos, G. V. Baron and J. F. M. Denayer, Separation of $\mathrm{CO}_{2} / \mathrm{CH}_{4}$ mixtures with the MIL-53 (Al) metal-organic framework, Microporous Mesoporous Mater., 2009, 120, 221-227, DOI: 10.1016/ j.micromeso.2008.11.007.

24 V. Finsy, H. Verelst, L. Alaerts, D. E. De Vos, P. A. Jacobs, G. V. Baron and J. F. M. Denayer, Pore-Filling-Dependent Selectivity Effects in the Vapor-Phase Separation of Xylene Isomers on the Metal-Organic Framework MIL-47, J. Am. Chem. Soc., 2008, 130, 7110-7118, DOI: 10.1021/ja800686c.

25 J. Alcañiz-Monge, G. Trautwein, M. Pérez-Cadenas and M. C. Román-Martínez, Effects of compression on the textural properties of porous solids, Microporous Mesoporous Mater., 2009, 126, 291-301, DOI: 10.1016/ j.micromeso.2009.06.020.

26 Y. H. Hu and L. Zhang, Amorphization of metal-organic framework MOF-5 at unusually low applied pressure, Phys. Rev. B: Condens. Matter Mater. Phys., 2010, 81, 174103, DOI: 10.1103/PhysRevB.81.174103.

27 Z. Jia, H. Li, Z. Yu, P. Wang and X. Fan, Densification of MOF-5 synthesized at ambient temperature for methane adsorption, Mater. Lett., 2011, 65, 2445-2447, DOI: 10.1016/j.matlet.2011.04.099.

28 J. J. Purewal, D. Liu, J. Yang, A. Sudik, D. J. Siegel, $\mathrm{S}$. Maurer and $\mathrm{U}$. $\mathrm{Mu}$, Increased volumetric hydrogen uptake of MOF-5 by powder densification, Int. J. Hydrogen Energy, 2012, 37, 2723-2727, DOI: 10.1016/ j.ijhydene.2011.03.002.

29 J. Purewal, D. Liu, A. Sudik, M. Veenstra, J. Yang, S. Maurer, U. Mu and D. J. Siegel, Improved Hydrogen Storage and Thermal Conductivity in High-Density MOF-5 
Composites, J. Phys. Chem. C, 2012, 116, 20199-20212, DOI: 10.1021/jp305524f.

30 D. Liu, J. J. Purewal, J. Yang, A. Sudik, S. Maurer, U. Mueller, J. Ni and D. J. Siegel, MOF-5 composites exhibiting improved thermal conductivity, Int. J. Hydrogen Energy, 2012, 37, 6109-6117, DOI: 10.1016/j.ijhydene.2011.12.129.

31 Y. Ming, J. Purewal, J. Yang, C. Xu, R. Soltis, J. Warner, M. Veenstra, M. Gaab, U. Mu and D. J. Siegel, Kinetic Stability of MOF-5 in Humid Environments: Impact of Powder Densification, Humidity Level, and Exposure Time, Langmuir, 2015, 31, 4988-4995, DOI: 10.1021/acs.langmuir.5b00833.

32 R. Zacharia, D. Cossement, L. Lafi and R. Chahine, Volumetric hydrogen sorption capacity of monoliths prepared by mechanical densification of MOF-177, J. Mater. Chem., 2010, 20, 2145-2151, DOI: 10.1039/B922991D.

33 A. Dailly and E. Poirier, Environmental Science Evaluation of an industrial pilot scale densified MOF-177 adsorbent as an on-board hydrogen storage medium, Energy Environ. Sci., 2011, 4, 3527-3534, DOI: 10.1039/C1EE01426A.

34 N. Heymans, S. Vaesen and G. De Weireld, A complete procedure for acidic gas separation by adsorption on MIL53 (Al), Microporous Mesoporous Mater., 2012, 154, 93-99, DOI: 10.1016/j.micromeso.2011.10.020.

35 M. A. Moreira, J. C. Santos, A. F. P. Ferreira, U. Müller, N. Trukhan, J. M. Loureiro and A. E. Rodrigues, Selective Liquid Phase Adsorption and Separation of ortho-Xylene with the Microporous MIL-53 (Al), Sep. Sci. Technol., 2011, 46, 1995-2003, DOI: 10.1080/01496395.2011.594481.

36 I. Majchrzak-kuce, Shaping metal-organic framework (MOF) powder materials for $\mathrm{CO}_{2}$ capture applications-a thermogravimetric study, J. Therm. Anal. Calorim., 2019, 138, 4139-4144, DOI: 10.1007/s10973-019-08314-5.

37 R. P. P. L. Ribeiro, C. L. Antunes, A. U. Garate, A. F. Portela, M. G. Plaza, J. P. B. Mota and I. A. A. C. Esteves, Binderless shaped metal-organic framework particles: impact on carbon dioxide adsorption, Microporous Mesoporous Mater., 2018, 275, 111-121, DOI: 10.1016/j.micromeso.2018.08.002.

38 K. W. Chapman, G. J. Halder and P. J. Chupas, PressureInduced Amorphization and Porosity Modification in a Metal-Organic Framework, J. Am. Chem. Soc., 2009, 131, 17546-17547, DOI: 10.1021/ja908415z.

39 D. Bazer-bachi, L. Assié, V. Lecocq, B. Harbuzaru and V. Falk, Towards industrial use of metal-organic framework: impact of shaping on the MOF properties, Powder Technol., 2014, 255, 52-59, DOI: 10.1016/j.powtec.2013.09.013.

40 J. Zhu, C. Dai, N. Yang, L. Jiang and Z. Lei, Gas adsorption in shaped zeolitic imidazolate framework-8, Chin. J. Chem. Eng., 2015, 23, 1275-1282, DOI: 10.1016/j.cjche.2015.01.015.

41 O. Ardelean, G. Blanita, G. Borodi, M. D. Lazar, I. Misan, I. Coldea and D. Lupu, Volumetric hydrogen adsorption capacity of densified MIL-101 monoliths, Int. J. Hydrogen Energy, 2013, 38, 7046-7055, DOI: 10.1016/j.ijhydene.2013.03.161.

42 H. Oh, D. Lupu, G. Blanita and M. Hirscher, Experimental assessment of physical upper limit for hydrogen storage capacity at $20 \mathrm{~K}$ in densified MIL-101 monoliths, RSC Adv., 2014, 4, 2648-2651, DOI: 10.1039/c3ra46233a.
43 G. Blanita, I. Coldea, I. Misan and D. Lupu, Hydrogen cryoadsorption by hexagonal prism monoliths of MIL-101, Int. J. Hydrogen Energy, 2014, 39, 17040-17046, DOI: 10.1016/ j.ijhydene.2014.08.038.

44 G. Blanita, M. Streza, M. D. Lazar and D. Lupu, Kinetics of hydrogen adsorption in MIL-101 single pellets, Int. J. Hydrogen Energy, 2017, 42, 3064-3077, DOI: 10.1016/ j.ijhydene.2017.01.010.

45 M. Tagliabue, C. Rizzo, R. Millini, P. D. C. Dietzel, R. Blom and S. Zanardi, Methane storage on CPO-27-Ni pellets, J. Porous Mater., 2011, 18, 289-296, DOI: 10.1007/s10934010-9378-0.

46 T. Remy, S. A. Peter, S. Van Der Perre, D. E. De Vos, G. V. Baron and J. F. M. Denayer, Selective Dynamic $\mathrm{CO}_{2}$ Separations on Mg-MOF-74 at Low Pressures: A Detailed Comparison with 13X, J. Phys. Chem. C, 2013, 117, 9301-9310, DOI: 10.1021/jp401923v.

47 G. W. Peterson, J. B. Decoste, T. G. Glover, Y. Huang, H. Jasuja and K. S. Walton, Effects of pelletization pressure on the physical and chemical properties of the metalorganic frameworks $\mathrm{Cu}_{3}(\mathrm{BTC})_{2}$ and UiO-66, Microporous Mesoporous Mater., 2013, 179, 48-53, DOI: 10.1016/ j.micromeso.2013.02.025.

48 J. Kim, S. Kim, S. Yang and W. Ahn, Bench-scale preparation of $\mathrm{Cu}_{3}(\mathrm{BTC})_{2}$ by ethanol reflux: Synthesis optimization and adsorption / catalytic applications, Microporous Mesoporous Mater., 2012, 161, 48-55, DOI: 10.1016/ j.micromeso.2012.05.021.

49 J. Dhainaut, J. Troyano, A. Legrand, J. Canivet, I. Imaz, D. Maspoch, H. Reinsch and D. Farrusseng, Systematic study of the impact of MOF densification into tablets on textural and mechanical properties, CrystEngComm, 2017, 19, 4211-4218, DOI: 10.1039/C7CE00338B.

50 A. Terracina, M. Todaro, M. Mazaj, F. M. Gelardi and G. Buscarino, Unveiled the Source of the Structural Instability of HKUST-1 Powders upon Mechanical Compaction: Definition of a Fully Preserving Tableting Method, J. Phys. Chem. C, 2019, 123, 1730-1741, DOI: 10.1021/ acs.jpcc.8b08846.

51 G. W. Peterson, J. B. Decoste, F. Fatollahi-fard and D. K. Britt, Engineering UiO-66-NH2 for Toxic Gas Removal, Nd. Eng. Chem. Res., 2014, 53, 701-707, DOI: 10.1021/ie403366d.

52 M. A. Moreira, C. Santos, A. F. P. Ferreira, J. M. Loureiro, F. Ragon, P. Horcajada, K. Shim, Y. Hwang, U. Lee, J. Chang, C. Serre and E. Rodrigues, Reverse Shape Selectivity in the Liquid-Phase Adsorption of Xylene Isomers in Zirconium Terephthalate MOF UiO-66, Langmuir, 2012, 28, 5715-5723, DOI: 10.1021/la3004118.

53 S. E. Bambalaza, H. W. Langmi, R. Mokaya, N. M. Musyoka and L. E. Khotseng, Compaction of a zirconium metalorganic framework (UiO-66) for high density hydrogen storage applications, J. Mater. Chem. A, 2018, 6, 23569-23577, DOI: 10.1039/C8TA09227C.

54 S. Edubilli and S. Gumma, A systematic evaluation of UiO66 metal organic framework for $\mathrm{CO}_{2} / \mathrm{N}_{2}$ separation, Sep. 
Purif. Technol., 2019, 224, 85-94, DOI: 10.1016/ j.seppur.2019.04.081.

55 Y. Khabzina, J. Dhainaut, M. Ahlhelm, H. Richter, H. Reinsch, N. Stock and D. Farrusseng, Synthesis and Shaping Scale-up Study of Functionalized UiO-66 MOF for Ammonia Air Purification Filters, Ind. Eng. Chem. Res., 2018, 57, 8200-8208, DOI: 10.1021/acs.iecr.8b00808.

56 J. Yang, H. Bai, F. Zhang, J. Liu, J. Winarta, Y. Wang and B. $\mathrm{Mu}$, Effects of Activation Temperature and Densification on Adsorption Performance of MOF MIL-100 (Cr), J. Chem. Eng. Data, 2019, 64, 5814-5823, DOI: 10.1021/ acs.jced.9b00770.

57 A. H. Valekar, K. Cho, U. Lee, S. Lee, W. Yoon, Y. K. Hwang, G. Lee, J. Cho and J. Chang, Shaping of porous metalorganic framework granules using mesoporous r-alumina as a binder, $R S C A d v ., 2017,7,55767-55777$, DOI: $10.1039 /$ C7RA11764G.

58 M. Taddei, M. J. McPherson, A. Gougsa, J. Lam and J. Sewell, An Optimised Compaction Process for $\mathrm{Zr}$ fumarate (MOF-801), Inorganics, 2019, 7, 110, DOI: 10.3390/inorganics7090110.

59 B. Kraushaar-Czarnetzki and S. P. Muller, in Shaping of Solid Catalysts, ed. K. P. de Jong, Synth. Solid Catal., 2009, pp. 173-199, DOI: 10.1002/9783527626854.ch9.

60 Y. Liu, D. Scharf and F. J. Clemens, Granulation processing parameters on the mechanical properties of diatomitebased porous granulates, Powder Technol., 2014, 263, 159-167, DOI: 10.1016/j.powtec.2014.04.094.

61 G. Liu, J. Guo, F. Meng, X. Zhang and L. Wang, Effects of Colloidal Silica Binder on Catalytic Activity and Adhesion of HZSM-5 Coatings for Structured Reactors, Chin. J. Chem. Eng., 2014, 22, 875-881, DOI: 10.1016/j.cjche.2014.06.010.

$62 \mathrm{X}$. Kong and J. Liu, Influence of Alumina Binder Content on Catalytic Performance of Ni/HZSM-5 for Hydrodeoxygenation of Cyclohexanone, PLoS One, 2014, 9, e101744, DOI: 10.1371/journal.pone.0101744.

63 M. S. Afarani, A. Samimi and E. B. Yekta, Synthesis of alumina granules by high shear mixer granulator: processing and sintering, Powder Technol., 2013, 237, 32-40, DOI: 10.1016/j.powtec.2013.01.007.

64 T. M. Chitu, D. Oulahna, M. Hemati, T. M. Chitu, D. Oulahna, M. H. Wet, T. M. Chitu, D. Oulahna and M. Hemati, Wet granulation in laboratory scale high shear mixers: Effect of binder properties, Powder Technol., 2011, 206, 25-33, DOI: 10.1016/j.powtec.2010.07.012.

65 G. T. Whiting, S. Chung, D. Stosic, A. D. Chowdhury, L. I. Van Der Wal, D. Fu, J. Zecevic, A. Travert, K. Houben, M. Baldus and B. M. Weckhuysen, Multiscale Mechanistic Insights of Shaped Catalyst Body Formulations and Their Impact on Catalytic Properties, ACS Catal., 2019, 9, 4792-4803, DOI: 10.1021/acscatal.9b00151.

66 P. Perez-Uriarte, M. Gamero, A. Ateka, M. Diaz, A. T. Aguayo and J. Bilbao, Effect of the Acidity of HZSM5 Zeolite and the Binder in the DME Transformation to Olefins, Ind. Eng. Chem. Res., 2016, 55, 1513-1521, DOI: 10.1021/acs.iecr.5b04477.
67 A. Permyakova, O. Skrylnyk, E. Courbon and M. Affram, Synthesis Optimization, Shaping, and Heat Reallocation Evaluation of the Hydrophilic Metal-Organic Framework MIL-160 (Al), ChemSusChem, 2017, 10, 1419-1426, DOI: 10.1002/cssc.201700164.

68 P. Kim, Y. You, H. Park, J. Chang, Y. Bae, C. Lee and J. Suh, Separation of $\mathrm{SF}_{6}$ from $\mathrm{SF}_{6} / \mathrm{N}_{2}$ mixture using metal-organic framework MIL-100 (Fe) granule, Chem. Eng. J., 2015, 262, 683-690, DOI: 10.1016/j.cej.2014.09.123.

69 V. D. Martins, R. A. Seabra, P. M. Silva, M. Ribeiro, K. H. Cho, U. Lee, J. Chang, M. Loureiro, A. E. Rodrigues and A. F. P. Ferreira, $\mathrm{C}_{2} / \mathrm{C}_{3}$ Hydrocarbon Separation by Pressure Swing Adsorption on MIL-100 (Fe), Ind. Eng. Chem. Res., 2020, 59, 10568-10582, DOI: 10.1021/ acs.iecr.0c00410.

70 P. Iacomi, U. Lee, A. H. Valekar, J. Chang and P. L. Llewellyn, Investigating the effect of alumina shaping on the sorption properties of promising metal-organic frameworks, RSC Adv., 2019, 9, 7128-7135, DOI: 10.1039/ c9ra00534j.

71 N. Chanut, A. D. Wiersum, U. Lee, K. Hwang, F. Ragon, H. Chevreau, S. Bourrelly, B. Kuchta, J. Chang, C. Serre and P. L. Llewellyn, Observing the Effects of Shaping on Gas Adsorption in Metal-Organic Frameworks, Eur. J. Inorg. Chem., 2016, 4416-4423, DOI: 10.1002/ejic.201600410.

72 S. Ohsaki, Y. Nakahara, H. Nakamura, S. Watano, M. Materials, Y. Nakahara, H. Nakamura and S. Watano, Flowability improvement of soft metal-organic framework particles by wet granulation, Microporous Mesoporous Mater., 2020, 293, 109785, DOI: 10.1016/j.micromeso.2019.109785.

73 J. Ren, N. M. Musyoka, H. W. Langmi, A. Swartbooi, B. C. North and M. Mathe, A more efficient way to shape metal-organic framework (MOF) powder materials for hydrogen storage applications, Int. J. Hydrogen Energy., 2015, 40, 4617-4622, DOI: 10.1016/j.ijhydene.2015.02.011.

74 S. Hindocha and S. Poulston, Study of the scale-up, formulation, ageing and ammonia adsorption capacity of MIL-100 (Fe), Cu-BTC and CPO-27 (Ni) for use in respiratory protection filters, Faraday Discuss, 2017, 201, 113-125, DOI: 10.1039/c7fd00090a.

75 M. J. Regufe, J. Tamajon, A. M. Ribeiro, A. F. P. Ferreira, U. Lee, Y. K. Hwang, J. Chang, C. Serre, J. M. Loureiro and A. E. Rodrigues, Syngas Purification by Porous AminoFunctionalized Titanium Terephthalate MIL-125, Energy Fuels, 2015, 29, 4654-4664, DOI: 10.1021/acs.energyfuels.5b00975.

76 M. Leloire, J. Dhainaut, P. Devaux, O. Leroy, H. Desjonqueres, S. Poirier, P. Nerisson, L. Cantrel, S. Royer, T. Loiseau and C. Volkringer, Stability and radioactive gaseous iodine-131 retention capacity of binderless UiO-66- $\mathrm{NH}_{2}$ granules under severe nuclear accidental conditions, J. Hazard. Mater., 2021, 416, 125890, DOI: 10.1016/j.jhazmat.2021.125890.

77 Z. Vajglova, N. Kumar, P. Arvela-Maki, K. Eranen, M. Peurla, L. Hupa, M. Nurmi, M. Toivakka and D. Y. Murzin, Synthesis and Physicochemical Characterization of Shaped Catalysts of $\beta$ and Y Zeolites for Cyclization of Citronellal, Ind. Eng. Chem. Res., 2019, 58, 18084-18096, DOI: 10.1021/acs.iecr.9b02829. 
78 P. Kusgens, A. Zgaverdea, H. Fritz, S. Siegle and S. Kaskel, Metal-Organic Frameworks in Monolithic Structures, J. Am. Ceram. Soc., 2010, 93, 2476-2479, DOI: 10.1111/ j.1551-2916.2010.03824.x.

79 G. Majano and J. Pérez-ramírez, Scalable RoomTemperature Conversion of Copper(II) Hydroxide into HKUST-1 $\left(\mathrm{Cu}_{3}(\mathrm{btc})_{2}\right)$, Adv. Mater., 2013, 25, 1052-1057, DOI: $10.1002 /$ adma.201203664.

80 W. Y. Hong, S. P. Perera and A. D. Burrows, Manufacturing of metal-organic framework monoliths and their application in $\mathrm{CO}_{2}$ adsorption, Microporous Mesoporous Mater., 2015, 214, 149-155, DOI: 10.1016/j.micromeso.2015.05.014.

81 W. Y. Hong, S. P. Perera and A. D. Burrows, Comparison of MIL-101 (Cr) metal-organic framework and 13X zeolite monoliths for $\mathrm{CO}_{2}$ capture, Microporous Mesoporous Mater., 2020, 308, 110525, DOI: 10.1016/j.micromeso.2020.110525.

82 C. A. Grande, V. I. Águeda, A. Spjelkavik and R. Blom, An efficient recipe for formulation of metal-organic frameworks, Chem. Eng. Sci., 2015, 124, 154-158, DOI: 10.1016/ j.ces.2014.06.048.

83 V. I. Agueda, J. A. Delgado, M. A. Uguina, P. Brea, A. I. Spjelkavik, R. Blom and C. Grande, Adsorption and diffusion of $\mathrm{H}_{2}, \mathrm{~N}_{2}, \mathrm{CO}, \mathrm{CH}_{4}$ and $\mathrm{CO}_{2}$ in UTSA-16 metalorganic framework extrudates, Chem. Eng. Sci., 2015, 124, 159-169, DOI: 10.1016/j.ces.2014.08.039.

84 J. A. Delgado, V. I. Águeda, M. A. Uguina, P. Brea and C. A. Grande, Comparison and evaluation of agglomerated MOFs in biohydrogen purification by means of pressure swing adsorption (PSA), Chem. Eng. J., 2017, 326, 117-129, DOI: 10.1016/j.cej.2017.05.144.

85 S. Pu, J. Wang, Z. Bao and Q. Yang, Performance Comparison of Metal-Organic Framework Extrudates and Commercial Zeolite for Ethylene/Ethane Separation, Ind. Eng. Chem. Res., 2018, 57, 1645-1654, DOI: 10.1021/ acs.iecr.7b04391.

86 J. Cousin-Saint-Remi, A. Finoulst, C. Jabbour, G. V. Baron and J. F. M. Denayer, Selection of binder recipes for the formulation of MOFs into resistant pellets for molecular separations by fixed-bed adsorption, Microporous Mesoporous Mater., 2020, 304, 109322, DOI: 10.1016/j.micromeso.2019.02.009.

87 M. Kriesten, V. Schmitz, J. Siegel, C. E. Smith, M. Kaspereit and M. Hartmann, Shaping of Flexible Metal-Organic Frameworks: Combining Macroscopic Stability and Framework Flexibility, Eur. J. Inorg. Chem., 2019, 4700-4709, DOI: 10.1002/ejic.201901100.

88 M. Klimakow, P. Klobes, A. F. Thunemann, K. Rademann and F. Emmerling, Mechanochemical Synthesis of MetalOrganic Frameworks: A Fast and Facile Approach toward Quantitative Yields and High Specific Surface Areas, Chem. Mater., 2010, 22, 5216-5221, DOI: 10.1021/cm1012119.

89 S. Tanaka, K. Kida, T. Nagaoka, T. Ota and Y. Miyake, Mechanochemical dry conversion of zinc oxide to zeolitic imidazolate framework, Chem. Commun., 2013, 49, 7884-7886, DOI: 10.1039/c3cc43028f.

90 A. M. Fidelli, B. Karadeniz, A. J. Howarth, I. Huskic, L. S. Germann, I. Halasz, M. Etter, S.-Y. Moon, R. E. Dinnebier,
V. Stilinovic, O. K. Farha, T. Friscic and K. Uzarevic, Green and rapid mechanosynthesis of high-porosity NU- and UiO-type metal-organic frameworks, Chem. Commun., 2018, 54, 6999-7002, DOI: 10.1039/C8CC03189D.

91 D. Lv, Y. Chen, Y. Li, R. Shi, H. Wu, X. Sun, J. Xiao, H. Xi, Q. Xia and Z. Li, Efficient Mechanochemical Synthesis of MOF-5 for Linear Alkanes Adsorption, J. Chem. Eng. Data., 2017, 62, 2030-2036, DOI: 10.1021/acs.jced.7b00049.

92 D. Crawford, J. Casaban, R. Haydon, N. Giri, T. McNally and S. L. James, Synthesis by extrusion: continuous, largescale preparation of MOFs using little or no solvent, Chem. Sci., 2015, 6, 1645-1649, DOI: 10.1039/c4sc03217a.

93 T. D. Ngo, A. Kashani, G. Imbalzano, K. T. Q. Nguyen and D. Hui, Additive manufacturing (3D printing): a review of materials, methods, applications and challenges, Composites, Part B, 2018, 143, 172-196, DOI: 10.1016/ j.compositesb.2018.02.012.

94 V. G. Rocha, E. Saiz, L. S. Tirichenko and E. García-Tuñón, Direct ink writing advances in multi-material structures for a sustainable future, J. Mater. Chem. A, 2020, 8, 15646-15657, DOI: 10.1039/D0TA04181E.

95 J. Azuaje, C. R. Tubío, L. Escalante, M. Gómez, F. Guitián, A. Coelho, O. Caamaño, A. Gil and E. Sotelo, An Efficient and Recyclable 3D Printed $\alpha-\mathrm{Al}_{2} \mathrm{O}_{3}$ Catalyst for the Multicomponent Assembly of Bioactive Heterocycles, Appl. Catal., A, 2017, 530, 203-210, DOI: 10.1016/j.apcata. 2016.11.031.

96 H. V. Thakkar, S. Eastman, A. Hajari, A. A. Rownaghi, J. C. Knox and F. Rezaei, 3D-Printed Zeolite Monoliths for CO Removal from Enclosed Environments, ACS Appl. Mater. Interfaces, 2016, 8, 27753-27761, DOI: 10.1021/ acsami.6b09647.

97 J. P. Lewicki, J. N. Rodriguez, C. Zhu, M. A. Worsley, A. S. Wu, Y. Kanarska, J. D. Horn, E. B. Duoss, J. M. Ortega, W. Elmer, R. Hensleigh, R. A. Fellini and M. J. King, 3D-Printing of Meso-structurally Ordered Carbon Fiber/Polymer Composites with Unprecedented Orthotropic Physical Properties, Sci. Rep., 2017, 7, 1-14, DOI: 10.1038/srep43401.

98 H. Thakkar, S. Eastman, A. Al-mamoori, A. Hajari, A. A. Rownaghi, F. Rezaei, H. Thakkar, S. Eastman, A. Almamoori, A. Hajari and A. A. Rownaghi, Formulation of Aminosilica Adsorbents into 3D-Printed Monoliths and Evaluation of their $\mathrm{CO}_{2}$ Capture Performance, ACS Appl. Mater. Interfaces., 2017, 9, 7489-7498, DOI: 10.1021/acsami.6b16732.

99 C. Parra-Cabrera, C. Achille, S. Kuhn, R. Ameloot and C. Parra-cabrera, 3D printing in chemical engineering and catalytic technology: structured catalysts, mixers and reactors, Chem. Soc. Rev., 2018, 47, 209-230, DOI: 10.1039/ C7CS00631D.

100 Z. Wang, J. Wang, M. Li, K. Sun and C. Liu, Threedimensional Printed Acrylonitrile with Cu-BTC Metalorganic Frameworks for the Removal of Methylene Blue, Sci. Rep., 2014, 4, 1-7, DOI: 10.1038/srep05939.

101 Z. Shi, C. Xu, F. Chen, Y. Wang, L. Li, Q. Meng and R. Zhang, Renewable metal-organic-frameworks-coated 
3D printing film for removal of malachite green, $R S C A d v$, 2017, 7, 49947-49952, DOI: 10.1039/c7ra10912a.

102 S. Lawson, Q. Al-naddaf, A. Krishnamurthy, M. S. Amour, C. Griffin, A. A. Rownaghi, J. C. Knox and F. Rezaei, UTSA16 Growth within 3D-Printed Co-Kaolin Monoliths with High Selectivity for $\mathrm{CO}_{2} / \mathrm{CH}_{4}, \mathrm{CO}_{2} / \mathrm{N}_{2}$, and $\mathrm{CO}_{2} / \mathrm{H}_{2}$ Separation, ACS Appl. Mater. Interfaces, 2018, 10, 19076-19086, DOI: 10.1021/acsami.8b05192.

103 D. Liu, P. Jiang, X. Li, J. Liu, L. Zhou, X. Wang and F. Zhou, 3D printing of metal-organic frameworks decorated hierarchical porous ceramics for high-efficiency catalytic degradation, Chem. Eng. J., 2020, 397, 125392, DOI: 10.1016/j.cej.2020.125392.

104 M. C. Kreider, J. A. Fedchak, J. Scherschligt, E. Abigail, N. Nikolai, Z. Ahmed and M. R. Hartings, Toward 3D printed hydrogen storage materials made with ABS-MOF composites, Polym. Adv. Technol., 2017, 29, 867-873, DOI: 10.1002/pat.4197.

105 M. Bible, M. Sefa, J. A. Fedchak, J. Scherschligt, B. Natarajan, Z. Ahmed and M. R. Hartings, 3D-Printed Acrylonitrile Butadiene Styrene-Metal Organic Framework Composite Materials and Their Gas Storage Properties, 3D Print, Addit. Manuf., 2018, 5, 63-72, DOI: 10.1089/3dp.2017.0067.

106 K. A. Evans, Z. C. Kennedy, B. W. Arey, J. F. Christ, H. T. Schaef, S. K. Nune and R. L. Erikson, Chemically Active, Porous 3D-Printed Thermoplastic Composites, ACS Appl. Mater. Interfaces, 2018, 10, 15112-15121, DOI: 10.1021/ acsami.7b17565.

107 H. Thakkar, S. Eastman, Q. Al-naddaf, A. A. Rownaghi and F. Rezaei, 3D-Printed Metal-Organic Framework Monoliths for Gas Adsorption Processes, ACS Appl. Mater. Interfaces, 2017, 9, 35908-35916, DOI: 10.1021/acsami.7b11626.

108 H. Thakkar, Q. Al-naddaf, N. Legion, M. Hovis, A. Krishnamurthy, A. A. Rownaghi and F. Rezaei, Adsorption of Ethane and Ethylene over 3D-Printed EthaneSelective Monoliths, ACS Sustain, Chem. Eng., 2018, 6, 15228-15237, DOI: 10.1021/acssuschemeng.8b03685.

109 S. Lawson, C. Gri, K. Rapp, A. A. Rownaghi and F. Rezaei, Amine-Functionalized MIL-101 Monoliths for $\mathrm{CO}_{2}$ Removal from Enclosed Environments, Energy Fuels, 2019, 33, 2399-2407, DOI: 10.1021/acs.energyfuels.8b04508.

110 S. Lawson, M. Snarzyk, D. Hanify, A. A. Rownaghi and F. Rezaei, Development of 3D-Printed Polymer-MOF Monoliths for $\mathrm{CO}_{2}$ Adsorption, Ind. Eng. Chem. Res., 2020, 59, 7151-7160, DOI: 10.1021/acs.iecr.9b05445.

111 S. Lawson, A. Alwakwak, A. A. Rownaghi and F. Rezaei, Gel-Print-Grow: A New Way of 3D Printing Metal-Organic Frameworks, ACS Appl. Mater. Interfaces, 2020, 12, 56108-56117, DOI: 10.1021/acsami.0c18720.

112 J. Lefevere, B. Claessens, S. Mullens, G. Baron, J. Cousinsaint-remi and J. F. M. Denayer, 3D-Printed Zeolitic Imidazolate Framework Structures for Adsorptive Separations, ACS Appl. Nano Mater., 2019, 2, 4991-4999, DOI: 10.1021/ acsanm.9b00934.

113 B. Claessens, N. Dubois, J. Lefevere, S. Mullens, J. Cousinsaint-remi and J. F. M. Denayer, 3D-Printed ZIF-8
Monoliths for Biobutanol Recovery, Ind. Eng. Chem. Res., 2020, 59, 8813-8824, DOI: 10.1021/acs.iecr.0c00453.

114 Z. Lyu, G. J. H. Lim, R. Guo, Z. Kou, T. Wang, C. Guan, J. Ding, W. Chen and J. Wang, 3D-Printed MOF-Derived Hierarchically Porous Frameworks for Practical HighEnergy Density $\mathrm{LiO}_{2}$ Batteries, Adv. Funct. Mater., 2019, 29, 1806658, DOI: 10.1002/adfm.201806658.

115 J. Dhainaut, M. Bonneau, R. Ueoka, K. Kanamori and S. Furukawa, Formulation of Metal-Organic Framework Inks for the 3D Printing of Robust Microporous Solids Towards High-Pressure Gas Storage and Separation, ACS Appl. Mater. Interfaces, 2020, 12, 10983-10992, DOI: 10.1021/acsami.9b22257.

116 R. Pei, L. Fan, F. Zhao, J. Xiao, Y. Yang, A. Lai, S. Zhou and G. Zhan, 3D-Printed metal-organic frameworks within biocompatible polymers as excellent adsorbents for organic dyes removal, J. Hazard. Mater., 2020, 384, 121418, DOI: 10.1016/j.jhazmat.2019.121418.

117 G. J. H. Lim, Y. Wu, B. B. Shah, J. J. Koh, C. K. Liu, D. Zhao, A. K. Cheetham, J. Wang and J. Ding, 3D-Printing of Pure Metal-Organic Framework Monoliths, ACS Mater. Lett., 2019, 1, 147-153, DOI: 10.1021/acsmaterialslett.9b00069.

118 C. A. Grande, R. Blom, V. Middelkoop, D. Matras, A. Vamvakeros, S. D. M. Jacques, A. M. Beale, M. Di, K. Anne and A. M. Bouzga, Multiscale investigation of adsorption properties of novel 3D printed UTSA-16 structures, Chem. Eng. J., 2020, 402, 126166, DOI: 10.1016/ j.cej.2020.126166.

119 S. Sultan, H. N. Abdelhamid, X. Zou and A. P. Mathew, CelloMOF: Nanocellulose Enabled 3D Printing of MetalOrganic Frameworks, Adv. Funct. Mater., 2018, 29, 1805372, DOI: $10.1002 /$ adfm.201805372.

120 A. J. Young, R. Guillet-Nicolas, E. S. Marshall, F. Kleitz, A. J. Goodhand, L. B. L. Glanville, M. R. Reithofer and J. M. Chin, Direct ink writing of catalytically active UiO-66 polymer composites, Chem. Commun., 2019, 55, 2190-2193, DOI: 10.1039/c8cc10018g.

121 O. Halevi, J. M. R. Tan, P. S. Lee and S. Magdassi, Hydrolytically Stable MOF in 3D-Printed Structures, Adv. Sustainable Syst., 2018, 2, 1700150, DOI: 10.1002/adsu.201700150.

122 W. Liu, O. Erol and D. H. Gracias, 3D Printing of an In Situ Grown MOF Hydrogel with Tunable Mechanical Properties, ACS Appl. Mater. Interfaces, 2020, 12, 33267-33275, DOI: 10.1021 /acsami.0c08880.

123 R. Li, S. Yuan, W. Zhang, H. Zheng and W. Zhu, 3D printing of mixed matrix films based on metal-organic frameworks and thermoplastic polyamide 12 by selective laser sintering for water applications, ACS Appl. Mater. Interfaces, 2019, 11, 40564-40574, DOI: 10.1021/acsami.9b11840.

124 E. Lahtinen, R. L. M. Precker, M. Lahtinen and E. Hey-hawkins, Selective Laser Sintering of Metal-Organic Frameworks: Production of Highly Porous Filters by 3D Printing onto a Polymeric Matrix, ChemPlusChem, 2019, 84, 222-225, DOI: 10.1002/cplu.201900081.

125 A. Gharsallaoui, G. Roudaut, O. Chambin, A. Voilley and R. Saurel, Applications of spray-drying in microencapsulation 
of food ingredients: An overview, Food Res. Int., 2007, 40, 1107-1121, DOI: 10.1016/j.foodres.2007.07.004.

126 A. Sosnik and K. P. Seremeta, Advantages and challenges of the spray-drying technology for the production of pure drug particles and drug-loaded polymeric carriers, Adv. Colloid Interface Sci., 2015, 223, 40-54, DOI: 10.1016/ j.cis.2015.05.003.

127 B. Vertruyen, N. Eshraghi, C. Piffet, J. Bodart, A. Mahmoud and F. Boschini, Spray-Drying of Electrode Materials for Lithium- and Sodium-Ion Batteries, Materials, 2018, 11, 1076, DOI: 10.3390/ma11071076.

128 J. Troyano, C. Ceren, L. Garzo, A. Carne, I. Imaz and D. Maspoch, Spray-Drying Synthesis of MOFs, COFs, and Related Composites, Acc. Chem. Res., 2020, 53, 1206-1217, DOI: 10.1021 acs.accounts.0c00133.

129 C. du Fresne von Hohenesche, V. Stathopoulos, K. K. Unger, A. Lind and M. Linden, Formation of hierarchically ordered silicas prepared by spray drying of nanosized spheres, Stud. Surf. Sci. Catal., 2002, 144, 339-346, DOI: 10.1016/S0167-2991(02)80153-7.

130 A. B. D. Nandiyanto, T. Ogi, W.-N. Wang, L. Gradon and K. Okuyama, Template-assisted spray-drying method for the fabrication of porous particles with tunable structures, Adv. Powder Technol., 2019, 30, 2908-2924, DOI: 10.1016/ j.apt.2019.08.037.

131 A. Carne-Sanchez, I. Imaz, M. Cano-Sarabia and D. Maspoch, A spray-drying strategy for synthesis of nanoscale metal-organic frameworks and their assembly into hollow superstructures, Nat. Chem., 2013, 5, 203-211, DOI: 10.1038/nchem.1569.

132 J. Huo, M. Brightwell, S. El Hankari, A. Garai and D. Bradshaw, A versatile, industrially relevant, aqueous room temperature synthesis of HKUST-1 with high spacetime yield, J. Mater. Chem. A, 2013, 1, 15220-15223, DOI: 10.1039/C3TA14409G.

133 A. Carné-Sánchez, K. C. Stylianou, C. Carbonell, M. Naderi, I. Imaz and D. Maspoch, Protecting Metal-Organic Framework Crystals from Hydrolytic Degradation by Spray-Dry Encapsulating Them into Polystyrene Microspheres, $A d v$. Mater., 2015, 27, 869-873, DOI: 10.1002/adma.201403827.

134 S. Tanaka and R. Miyashita, Aqueous-System-Enabled Spray-Drying Technique for the Synthesis of Hollow Polycrystalline ZIF-8 MOF Particles, ACS Omega, 2017, 2, 6437-6445, DOI: 10.1021/acsomega.7b01325.

135 Y.-R. Lee, M.-S. Jang, H.-Y. Cho, H.-J. Kwon, S. Kim and W.S. Ahn, ZIF-8: a comparison of synthesis methods, Chem. Eng. J., 2015, 271, 276-280, DOI: 10.1016/j.cej.2015.02.094.

136 S. Chaemchuen, K. Zhou, B. Mousavi, M. Ghadamyari, P. M. Heynderickx, S. Zhuiykov, M. S. Yusubov and F. Verpoort, Spray drying of zeolitic imidazolate frameworks: investigation of crystal formation and properties, CrystEngComm, 2018, 20, 3601-3608, DOI: 10.1039/C8CE00392K.

137 Y. Mitsuka, K. Nagashima, H. Kobayashi and H. Kitagawa, A Seed-mediated Spray-drying Method for Facile Syntheses of Zr-MOF and a Pillared-layer-type MOF, Chem. Lett., 2016, 45, 1313-1315, DOI: 10.1246/cl.160651.
138 L. Garzón-Tovar, M. Cano-Sarabia, C. Carbonell, I. Imaz and D. Maspoch, A spray-drying continuous-flow method for simultaneous synthesis and shaping of microspherical high nuclearity MOF beads, React. Chem. Eng., 2016, 1, 533-539, DOI: 10.1039/C6RE00065G.

139 S. Bae, N. Zaini, K. S. N. Kamarudin, K. S. Yoo, J. Kim and M. Roslee, Rapid solvothermal synthesis of microporous UiO66 particles for carbon dioxide capture, Korean J. Chem. Eng., 2018, 35, 764-769, DOI: 10.1007/s11814-017-0334-4.

140 M. J. Katz, Z. J. Brown, Y. J. Colon, P. W. Siu, K. A. Scheidt, R. Q. Snurr, J. T. Hupp and O. K. Farha, A facile synthesis of UiO-66, UiO-67 and their derivatives, Chem. Commun., 2013, 49, 9449-9451, DOI: 10.1039/C3CC46105J.

141 C. Avci-Camur, J. Troyano, J. Pérez-Carvajal, A. Legrand, D. Farrusseng, I. Imaz and D. Maspoch, Aqueous production of spherical Zr-MOF beads via continuous-flow spraydrying, Green Chem., 2018, 20, 873-878, DOI: 10.1039/ C7GC03132G.

142 G. C. Shearer, S. Chavan, S. Bordiga, S. Svelle, U. Olsbye and K. P. Lillerud, Defect Engineering: Tuning the Porosity and Composition of the Metal-Organic Framework UiO66 via Modulated Synthesis, Chem. Mater., 2016, 28, 3749-3761, DOI: 10.1021/acs.chemmater.6b00602.

143 G. Boix, J. Troyano, L. Carlos, G. Tovar, C. Camur, N. Bermejo, A. Yazdi, J. Piella, N. G. Bastús, V. Puntes, I. Imaz and D. Maspoch, MOF-Beads containing inorganic nanoparticles for the simultaneous removal of multiple heavy metals from water, ACS Appl. Mater. Interfaces, 2020, 12, 10554-10562, DOI: 10.1021/acsami.9b23206.

144 G. Boix, X. Han, I. Imaz and D. Maspoch, MillimeterShaped Metal-Organic Framework/Inorganic Nanoparticle Composite as a New Adsorbent for Home WaterPurification Filters, ACS Appl. Mater. Interfaces., 2021, 13, 17835-17843, DOI: 10.1021/acsami.1c02940.

145 A. I. Spjelkavik, S. Divekar, T. Didriksen and R. Blom, Forming MOFs into Spheres by Use of Molecular Gastronomy Methods, Chem. - Eur. J., 2014, 20, 1-7, DOI: 10.1002/chem.201402464.

146 D. W. Lee, T. Didriksen, U. Olsbye, R. Blom and C. A. Grande, Shaping of metal-organic framework UiO-66 using alginates: Effect of operation variables, Sep. Purif. Technol., 2020, 235, 116182, DOI: 10.1016/j.seppur.2019.116182.

147 D. Farrusseng, C. Daniel, C. Hamill, J. Casaban, T. Didriksen, R. Blom, A. Velte, G. Fueldner, P. Gantenbein, P. Persdorf, $X$. Daguenet-Frick and F. Meunier, Adsorber heat exchanger using Al-fumarate beads for heat-pump application - a transport study, Faraday Discuss, 2020, 225, 384-402, DOI: 10.1039/ D0FD00009D.

148 S. Yang, L. Peng, O. A. Syzgantseva, O. Trukhina, I. Kochetygov, A. Justin, D. T. Sun, H. Abedini, M. A. Syzgantseva, E. Oveisi, G. Lu and W. L. Queen, Preparation of Highly Porous Metal-Organic Framework Beads for Metal Extraction from Liquid Streams, J. Am. Chem. Soc., 2020, 142, 13415-13425, DOI: 10.1021/jacs.0c02371.

149 X.-X. Liang, N. Wang, Y. Qu, L.-Y. Yang, Y.-G. Wang and X.-K. Ouyang, Facile Preparation of Metal-Organic Framework 
(MIL-125)/Chitosan Beads for Adsorption of Pb(II) from Aqueous Solutions, Molecules, 2018, 23, 1524, DOI: 10.3390/ molecules23071524.

150 A. M. Omer, E. M. A. El-monaem, M. M. A. El-latif, G. M. Elsubruiti and A. S. Eltaweil, Facile fabrication of novel magnetic ZIF-67 MOF@aminated chitosan composite beads for the adsorptive removal of $\mathrm{Cr}(\mathrm{VI})$ from aqueous solutions, Carbohydr. Polym., 2021, 265, 118084, DOI: 10.1016/j.carbpol.2021.118084.

151 B. J. Riley, W. Kuang, T. Varga, S. Ahmed, M. Galanek, J. Li, Z. Nelson and P. K. Thallapally, Metal-organic frameworkpolyacrylonitrile composite beads for xenon capture, ACS Appl. Mater. Interfaces., 2020, 12, 45342-45350, DOI: 10.1021/acsami.0c13717.

152 J. Park, Y. S. Chae, D. W. Kang, M. Kang, J. H. Choe, S. Kim, J. Y. Kim, Y. W. Jeong and C. S. Hong, Shaping of a Metal-Organic Framework - Polymer Composite and Its $\mathrm{CO}_{2}$ Adsorption Performances from Humid Indoor Air, ACS Appl. Mater. Interfaces., 2021, 13, 25421-25427, DOI: 10.1021/acsami.1c06089.

153 S. Gökpinar, S. Ernst, E. Hastürk, M. Möllers, E. Aita, R. Wiedey, N. Tannert, S. Nießing, S. Abdpour, J. Quodbach, G. Füldner, S. K. Henninger and C. Janiak, Air-con Metal-Organic Frameworks in Binder Composites for Water Adsorption Heat Transformation Systems, Ind. Eng. Chem. Res., 2019, 58, 21493-21503, DOI: 10.1021/ acs.iecr.9b04394.

154 E. Hastürk, C. Schlüsener, J. Quodbach, A. Schmitz and C. Janiak, Shaping of metal-organic frameworks into mechanically stable monoliths with poly(vinyl alcohol) by phase separation technique, Microporous Mesoporous Mater., 2019, 280, 277-287, DOI: 10.1016/j.micromeso.2019.02.011.

155 Y. Hara, K. Kanamori and K. Nakanishi, Self-Assembly of Metal-Organic Frameworks into Monolithic Materials with Highly Controlled Trimodal Pore Structures, Angew. Chem., Int. Ed., 2019, 58, 19047-19053, DOI: 10.1002/anie.201911499.

156 A. Ahmed, T. Hasell, R. Clowes, P. Myers, A. I. Cooper and H. Zhang, Aligned macroporous monoliths with intrinsic microporosity via a frozen-solvent-templating approach, Chem. Commun., 2015, 51, 1717-1720, DOI: 10.1039/ C4CC08919G.

157 Q. Fu, L. Wen, L. Zhang, X. Chen, D. Pun, A. Ahmed, Y. Yang and H. Zhang, Preparation of Ice-templated MOFpolymer composite monoliths and their application for wastewater treatment with high capacity and easy recycling, ACS Appl. Mater. Interfaces, 2017, 9, 33979-33988, DOI: $10.1021 /$ acsami.7b10872.

158 Q. Fu, L. Zhang, H. Zhang, X. Chen, M. Li and M. Gong, Ice- and MOF-templated porous carbonaceous monoliths for adsorptive removal of dyes in water with easy recycling, Environ. Res., 2020, 186, 109608, DOI: 10.1016/j.envres. 2020.109608.

159 Q. Fu, L. Wen, L. Zhang, X. Chen and H. Zhang, Porous Carbon and Carbon/Metal Oxide Composites by Ice Templating and Subsequent Pyrolysis, Ind. Eng. Chem. Res., 2019, 58, 14312-14322, DOI: 10.1021/acs.iecr.9b01081.

160 T. Tian, J. Velazquez-Garcia, T. D. Bennett and D. FairenJimenez, Mechanically and chemically robust ZIF-8 monoliths with high volumetric adsorption capacity, J. Mater. Chem. A, 2015, 3, 2999-3005, DOI: 10.1039/c4ta05116e. 